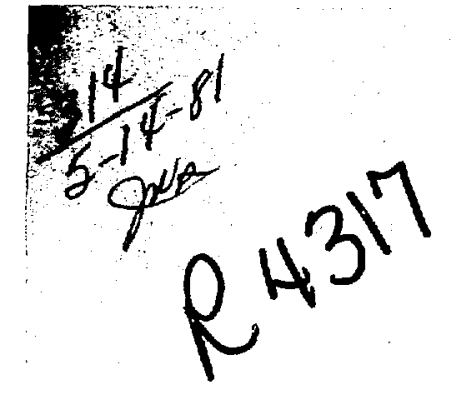

佔. 2647

\title{
MASTER
}

LBL-1 1895

UC-70

\section{THE MEASUREMENT OF IN-SITU STRESS IN SALT AND ROCK USING NQR TECHNIQUES}

\author{
E. Schempp, T. Hirschfeld, and S. Klainer
}

December 1980

Prepared for the U.S. Department of Energy

under Contract W.7405.ENG-48

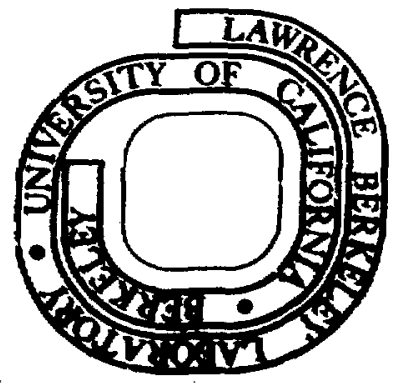


SURMARY

This Annual Report presents a summary of the first four months' results from investigating the potential for measuring stress/ train in salt using NQR techniques.

This investlgation is based on the premise that there exists 1 need for an improved method of measuring underground stress -- a method which would be reliable, simple to use, relatively fast, non-destructive, and inexpensive. A detailed discussion of how stress and strain affect the quantities which can be measured in an NQR experiment is then given. "is shows that, for stresses of the magnitude to be expected at depths up to about 10,000 feet, quadrupole coupling constants will fall in the range of 1-10 $\mathrm{kHz}$ for both the sodium and chloride fons in $\mathrm{MaCl}$. The report continues with an examination of various resonance techniques which inight be utilized to carry out the measurenents. The nost promising system involves pulsed nuclear dobile resonance detection; an alterative is to observe the quadrupolar splitting of the NMR signal. (For studies of aluminum in hard rock, a later phase of this program, the 127 NQR lines would most likely De detected by pure quadrupole resonance.)

an extensive discussion is presented on the choices to be made in the neasurenert and riapping techniques. The well-known perturbation of the nuldogenous stress field in the neighborhood of a borehole is shown to be advantageous from the point of view of obtaining directional information on 
the stress. Detailed considerations are then given for the construction and operation of a borehole stress sensor.

The important conclusions reached to date are: (1) The NQR techrique seems feasible for weasuring the magnitude and direction of underground stress witn a resolution of about $25 \mathrm{psi}$, or $2.5 \%$ at $1000 \overline{0}$ pi. Downhole instrumentation suitable for in-situ determinations of stress appesirs within the state of the art.

Based on the work to date, the authors have 1dentified additional rasks required on the project. These include detailed laboratory measurements on salt as a function of pressure and direction, continued theoretical calculation on the exact line shapes to be expected, and the contribution of lattice defects and impurities to the NQR signals. Finalizing the design of a laboratory NQR 1nstrument and constructing it will also be part of the program for the present fiscal year. It will later be adapted it to a field configuration for in-situ measurements in salt. Preliminary investigation of the NQR signals from Al-containing mineral constituents of hard rock will be carrled out in parallel with the above work program. 
THE MEASUREMENT OF IN-SITU STRESS IN SALT AND ROCK USING NQR TECHNIQUES

Table of Contents

I. Introduction .................... 1

A. Overview ........................ 1

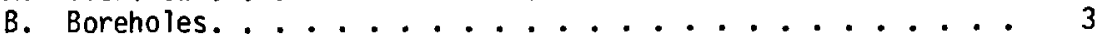

1. The Leeman Rosette Gauge ("doorstopper") . . . . . . . . 5

2. Enclosed Probes. ................. 5

3. Hydraulic Fracturing ................ 5

C. Program Plan ..................... . . 7

D. Prognosis....................... 7

II. Technical Discussion .................. 9

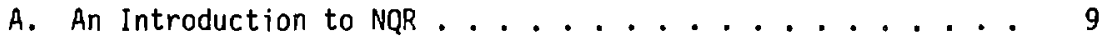

B. NQR and Strain Measurements. ............. 12

C. Resonance Techniques ................ 24

1. Direct Detection .............. 24

2. Quadrupolar Perturbed NMR. . . . . . . . . . . 26

3. Double Resonance ................. 29

D. Choice of Measurement Techn ique. ......... 35

1. Choice of Measurement Technique.......... 35

2. Choice of Mapping Method ........... 37

3. Mapping of the Surrounding Strata by NQR . . . . . . 37

4. Mapping the Borehole Perturbation by NQR ....... 44

E. Description of an NQR Stress Sensor for Borehole

Operation ........................ 48

1. Magnetic Field Generator ............. 48

a. Field solenoid............... 48

b. Power supply.............. 53

c. Cooling system ............ 55

2. NMR/NQR Double Resonance Transmitter-Receiver..... 55

a. NQR irradiator ............. 55

b. A rotating transmit/receive coil ...... 56

c. A matched switching network and preamplifier.... 58

d. NMR transmitter/receiver system. . . . . . . . 64

e. Data system. . . . . . . . . . . . 65

F. Predicted Sensitivities on Stress/Strain Determinations. . 66

III. Conclusions and Recommendations. ............ 77

A. Conclusions................... 77

B. Recormendations. ............... 77

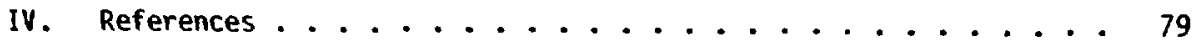


V. Appendices

A. Practical Applications of FT-NQR Spectroscopy. . . . . . A-1

B. Axial Field of a Coil of Finite Height and Width . . . . . . B-1

C. Effect of Finite Winding Height on Axial Field of

Toroidal Coil. ...................... C-1

D. Effect of Finite Annular Width on Axial Field of

Toroidal Coil................ D-1

E. Optimum NQR Coil for Coristant Maximum Field. . . . . . . E-1

G. Power Requirements in NQR Coil............ . G-1

H. NQR Signal Loss for Finite Length Excitation Pulses. . . . H-1 
THE MEASUREMENT OF IN-SITU

STRESS IN SALT AND ROCK USING

NQQR TECHNIQUES

\section{INTRODUCTION}

\section{A. Overview}

The measurement of the in situ state of stress underground is onc of the most challenging and important problems presented to geoscience and engineering. Upon accurate knowledge of the stress and the strength of rock materiais rests all designs for both surface structure and underground excavations. A special case of this need is the measurement of stress in rock and/or salt to assure the integrity of an underground nuclear waste repository. Knowledge of present, and if possible past, stresses are important in developing a deeper understanding of the forces which have shaped and are continuing to shape the earth's landforms. In a time of ever greater human demands on the earth's crustal resources, such a broader understanding is increasingly vital. Moreover, growing population densities place more and more people in areas subject to risk from earthquakes, volcanoes, and other geological forces which can dramatically affect lives and economies. If improved techniques for making more numerous and more accurate stress measurements can be discovered, our knowledge ind ability to predict the structural soundness of excavations in rock and sait will be greatly aided. 
In spite of the fundamental role played by stress, techniques and instrumentation for its measurement have developed slowly. In some of the most frequently used techniques it is necessary to interrupt the drilling operation to carry out the measurements, a procedure which is obviously costly. Other methods are both costly and destructive. The technology for making rapid, non-destructive stress determinations does not exist. No existing methods are available for carrying out ineasurinents at a particular depth in a borehole over a period of time, and hence observations of changes in stress over time are almost unobtainable. Clearly, in areas where knowledge of sudden build-ups or release of stress would be important, such as in earthquake and fault zones and near waste repositories and volcanoes, an improved more versatile technique for determining stress would be very valuable.

It is the purpose of this research work to examine the feasibility of using NQR (Nuclear Quadrupole Resonance) techniques, a new procedure for measuring stress, and to develop the instrumentation necessary to permit field evaluation.

Although several concepts are under evaluation which are all based on the microscopic response of crystalline materials to stress and utilize sophisticated notions drawn from experience in solid state physics, the present total emphasis is on NQR. The approaches being considered include changes in the nuclear spin-lattice relaxation times of NMR signals, changes in the olectronspin resonance (ESR) signals from iron-bearing minerals in rock, and the pressure-dependence of nuclear quadrupole resonance (NQR) signals. The focus of this report is on salt, $\mathrm{NaCl}$, which while normally cubic, under stress changes its symmetry and thus develops a NQR spectrum which depends on the stress-induced strains. In the following pages the theoretical and 
experimental details are described in ful1. Once the salt studies have demonstrated the feasibility of the technique, then the use of NQR to determine stress in aluminum-containing rocks will be addressed.

\section{B. Boreholes}

Knowledge of the in situ state of stress is important in the design of underground openings. The stability, safety, and long-term behavior of underground structures depends largely on the properties of the rock found at the site of the proposed works, and good design requires accurate knowledge of the relevant parameters. The overall mechanical properties of rock depend upon all of the structural features and the state of stress in the rocks throughout the site. Whenever the stresses exceed the relevant materials strengths, the design of the layout must be modified to obviate this situation or the rock must be reinforced to ensure stability.

Boreholes drilled to penetrate the rock mass provide the most reliable way, short of full-scale excavation, of obtaining information on the rocks at depth and it is, therefore, from boreholes that one would like to be able to measure the stress.

Rocks underground are subject to a variety of forces. The most obvious is the lithostatic pressure from the weight of the overburden. Other forces include those from tectonic movements, i.e., horizontal or sideways thrusts of the type responsible for uplift, folding, and faulting. In principle, the separate vector forces can be added to give a resultant force, and then from this a net pressure (force per unit area) acting in a known direction can be derived. This simple approach, however, does not take into account the deformability of materials. Owing to strains, dimensional changes arising 
from stress, a simple vertical pressure will be converted into horizontal forces. Hence one prefers to analyze the situation in terms of the stress tensor. In general, therefore, rocks are subjected to a 3-dimensional stress made up of both diagonal (normal) elements, which are usually compressive, and off-diagonal shear elements. A complete specification of the tensor requires knowing all six elements, or the three diagonal elements in the principal axes system and the three angles which specify the orientation of the principal axes. For a full description of the philosophical origins of the concept of stress and its detailed mathematical treatment, the reader is referred to standard works on the theory of eiasticity, e.g., the one by Love. (1)

One probelm presented by the concept of underground stress is that the quantity of interest is not directly accessible to measurement. The reason for this is that in order to get to the point where a measurement is desired, a hole has to be made, and the hole itself perturbs the stress field. There can, of course, be no normal stress on a surface facing the opening. In most cases, where the stress is reasonably homogeneous and the rock can be considered a continuous medium, the stress in the region of the borehole can be calculated using the Laplace equation. Thus from measurements of the radial and tarigential components of the stress near the borehole, the Cartesian components present before the hole was drilled can be calculated.

A large number of techniques have been devised to measure stress. Three of these, indely used in borehole situations, will be briefly described. A number of other methods are discussed by Jaeger and Cook. (2) 
1) The Leeman Rosette Gauge ("doorstopper") (3)

In this technique, a hole is drilled to the depth of interest. The main drilling bit is then pulled out, and a small pilot hole about 1 or 2 inches in diameter is drilled an additional 10-20 inches. The bottom of this hole is carefully faced to be smooth and flat. Then three rosettes of three strain gauges each is inserted and cemented directly to the surface of the rock at the botton of the borehole. After the cement has dried, electrical measurements are made, the connecting cables are disconnected, the main coring bit is re-lowered, and the pilot hole with the implanted strain gauge is overcored, thus releasing the stress from the surrounding rock. When the core is brought to the surface, a new set of measurements from the strain gauges is taken. This scheme represents a significant improvement over previous methods in that one set of measurements from a single borehole provides sufficient data to enable the complete state of stress to be determined at the measurement point.

2) Enclosed Probes

Rocha and Silverio ${ }^{(4)}$ and Blackwood ${ }^{(5)}$ developed a second technique in which the strain gauges are imbedded in a solid epoxy probe. This is bonded into the pilot hole and overcored as above. Later Pender and Duncan-Fama ${ }^{(6)}$ developed and analyzed an improved technique utilizing a thin-walled epoxy cylinder in which the strain gauges were placed.

3) Hydraulic Fracturing

In this meth $d$ a section of the borehole is sealed off by two packers and the region between them pumped full of water at high pressure. The pressure is increased unti] the rock walls fracture, and then by measuring the subsequent "shut-in pressure" and "secondary breakdown pressures" parameters are obtained from which the stress may be inferred, if the pore pressure and 
hydrofracture tensile strength of the rock is known from laboratory measurements a priori. The directions of the stress are obtained by observing the directions of the cracks produced in the borehole wall, either by lowering a camera or some device for making impressions of the wall surfaces down the hole.

It is apparent that all these methods are relatively cumbersome and timeconsuming. The first two using triaxial strain gauges require that the drilling operation be interrupted, the pilot hole drilled, the probe inserted, and the overcoring drilling step. It is obviously difficult to repeat this at frequent intervals. Morenver, they cannot be applied to pre-existing holes. Gther problems are present: (a) the Leeman "doorstopper" technique is not very satisfactory under wet conditions. (b) The epoxy probes may give wrong readings if the bond between the rock and the probe is broken by tensile stresses introduced by overcoring and (c) the hydrofracture method is, to say the least, an indirect way of obtaining the stress; it involves considerable skill in setting up the experiment; it requires laboratory studies on the rocks from the region of the borehole; and, of course, it also inflicts permanent damage on the walls of the borehole. None of these methods is suitable for measuring the time evolution of the stress because the measurements cannot be repeated at a later time.

in contrast, the NQR method offers the possibility of lowering an instrument down a borehole in salt and making as many measurements as desired at arbitrary increments in depth, and these can be repeated as of ten as necessary. Each measurement should be completed in a half hour or less.

Underground stresses vary over a wide range with values as large as 10,000 psi $(680 \mathrm{~atm})$ having been reported. (2) The majority of measursments, however, fall under 2500 psi (140 atm), particularly for lighter rock types 
such as salt. (7) At depth, all rock is subjected to a vertical lithostatic stress, and it is frequently assumed that at great depth (>1000 $\mathrm{m}$ ) it is only this stress which matters. This idea has been called Heirin's rule. (2) This situation gives rise to horizontal stresses of the same order as the vertical, although many situations are known where the horizontal stresses $\sigma_{1}$ and $\sigma_{2}$ are higher than the vertical. Often, the two horizontal components of the stress tensor obey $\sigma_{1} / \sigma_{2} \simeq 0.5$.

\section{Program Plan}

The overall program objectives are to demonstrate that in-situ stress can be measured in salt and aluminum-contairing rocks using NQR techniques. The inves:igation has been initiated using salt because this represents a simple, i.e., essentially one species, natural system. Once feasibility in salt has been demonstrated with optimized laboratory measirements, the study plan calls for two parallel programs: (a) in-situ stress measurements of salt and (b) development of techniques for determining stress in. ocks. The details of the antic:pated plan are shown in Table 1.

\section{Prognosis}

It was recognized, when this program was initiatea, that there were many unanswered questions regarding the ability of NQR spectroscopy to provide stress information which was relevant to the rock mechanics experts. In particular there was the question of converting spectra' data to useable mechanicai information. Furthermore, there were questions as to what stress 
Table 1. Anticipated Program Plan for NQR Measurements of St.ress/Strain in Rocks

\begin{tabular}{|c|c|c|c|c|c|c|}
\hline rask & FY 10 & FY 81 & FY 12 & Fr 03 & FY 8 & $F$ \\
\hline 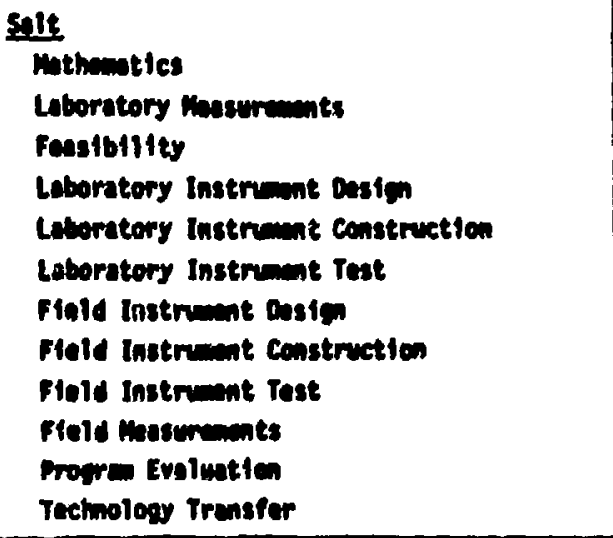 & & & & $\Rightarrow$ & & \\
\hline 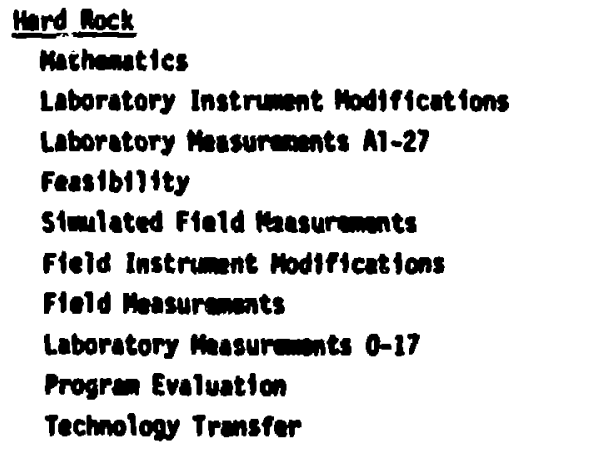 & & ם & $\Delta$ & & $\square$ & $\overline{\mathbf{J}}$ \\
\hline
\end{tabular}


jifferences were resolvable and if instrumentation existed, or could be conceptually designed, which would have the capability of making the prescribed measurements.

LBL has not underestimated the difficulty of accomplishing the proposed objectives. To reach our goals requires funcamental research in both physics and instrumentation engineering. It alsc involves the synthesis of skills from several technical disciplines. It has not been possible to answer all questions or solve all problems in the first four monchs. However, considering the slow progress with which other stress measuring techniques have developed, outstanding progress has been made. The results are favorable and success of the program now appears within reach. Examination of the physics, spectroscopy, rock mechanics and (instrumental) engineering indicate there were no flaws in the original postulations. Calculations using spectral signal-to-noise indicates that three-dimentional pressures of 1.5 to 10 psi can be resolved.

\section{TECHNICAL DISCUSSION}

\section{A. An Introduction to NQR}

Nuclear quadrupole resonance (NQR) is a radio-frequency spectroscopic technique which, like nuclear magnetic resonance (NMR) uses the nucleus as a small, highly specific, probe of its environment. In NQR, one employs certain nuclei which possess electric quadrupole moments (those with spin I $\geq 1$ ) to study the electric fields with which they interact, or more specifically the electric fieid gradient (EFG) tensor. The EFG, in turn, varies in relation to 
all the chemistry and physics in the crystal including the bonding configuration and distribution of valence electrons, the crystallographic site symmetry, crystal defects such as dislocations and impurities, and strains arising from both internal and external stress. Because the EFG is so very sensitive to the state of the lattice and because NQR is a resonance spectroscopic technique, it is of ten possible to see variations of one part in $10^{5}$ or better. An overview of modern NQR spectrocopy appears in Appendix A.

Most details of the crystal lattice and chemistry express themselves primarily in terms of electrostatics, which NQR senses directly (the local magnetic fields studied in NMR are usually only a secondary consequence of changes experienced first in the EFG). Two other features serve to make NQR attractive to studies in geochemistry: (1) NQR is applied to solids--single crystals, polycrystalline materials, or amorohous materials (it does not work in liquids), and (2) MNR is suitable for field studies since it does not require a large heavy magnet as does NMR because the field parameter, the EFG, is intrinsic to the material.

In the studies undertaken as part of this project the focus has been on determining strain in rock salt, $\mathrm{NaCl}$. In unstrained salt, both the sodium and chloride ions occlipy sites of cubic symmetry, which by very general principles cannot possess an EFG. Thus the quadrupole coupling is zero. However, when subjected to non-isostatic stress, the crystal will deform producing non-cubic site symmetries at the $\mathrm{Cl}$ atom positions, thus producing a small EFG. For example, a compressive stress along [100] will make the crystal tetragonal, a stress applied 3long [110] makes the crystal orthorhombic, a stress along [111] changes the crystal to the trigonal class, etc. The field 
gradient must reflect the site symmetry of the lattice. Experiments by Marsh and Casabella ${ }^{(8)}$ indicate that the $N Q R$ frequency shift for $\mathrm{Cl}^{35}$ in $\mathrm{NaCl}$ is on the order of $30 \mathrm{~Hz} / \mathrm{atm}$.

The field gradient is not observed directly, but in the product $e^{2} \mathrm{qQ}$ where $\mathrm{eQ}$ is the nuclear quadrupole moment and is known a priori and eq is the maximum component of the EFG in the coordinate system in which the tensor is diagonal; $e^{2} q Q$ is known as the quadrupole coupling constant and is usually expressed in units of frequency by dividing by Planck's constant, $h$. The existence of a non-zero $\mathrm{e}^{2} \mathrm{qQ}$ means that the nuclei in the crystal will have a preferred orientation in space, and each orientation will be associated with a derinite energy. (9) Owing to the quantum mechanical spin properties of nuclei, only certain orientations are allowed, giving rise to discrete energy levels. The nuclei in a solid can be made to absorb quanta of rf radiation as they "flip" between these energy levels. This is the phenomenon of resonance. In the case of $\mathrm{Na}^{23}$ and $\mathrm{Cl}^{35}$, both of which possess nuclear spin $3 / 2$, the resonance frequency $v$ is related to the quadrupole coupling constant via

$$
\nu=\frac{1}{2} \mathrm{e}^{2} \mathrm{qQ}\left(1+\frac{\eta^{2}}{3}\right)^{1 / 2}
$$

$\eta$ is a quantity which depends on the departure of the EFG from axial symmetry. In the case of stressed salt, a measurement of $v$ determines $q$ which in turn depends on the stress.

When $\mathrm{e}^{2} \mathrm{qQ}$ is small, less than about $0.5 \mathrm{MHz}$, the pure quadrupole resonance is usually not observed directly because the sensitivity of detection becomes small. In this case, the quadrupolar coupling ran be detected as 
a perturbation on the NQR signal or by use of so-called double resonance techniques. The mathmematical and experimental details are described in the following sections.

\section{B. NQR and Strain Measurements}

From the theory of elasticity in linear media stressed below the elastic imit, the strain $E$ is related to the stress o by the compliances $s$, giving Hooke's law in three-dimensions as

$$
\epsilon_{\mathrm{ij}}=s_{\mathrm{ijkl}} o_{\mathrm{k} 1}
$$

(where the summation convention is used, meaning that a $\Sigma$ over all repeated indices in an expression is to be understood). Although the $\dot{s}_{i j k l}$ form the components of a 4th rank tensor and thus consist of 81 separate elements, energy conservation considerations reduce the number to 36 independent elements in the general case, and in the cubic system, which concerns us here, to only three independent components $s_{1111}, s_{1122}$, and $s_{2323}$ (Nye). (10) In the nearly universally used matrix notation in which the indices are condensed and summed from 1 to 6 , we have

$$
\epsilon_{\mathrm{i}}=s_{\mathrm{ij}} \sigma_{\mathrm{j}}
$$


with non-vanishing components in the $\mathrm{NaCl}$ class m3m

$$
\begin{aligned}
& s_{11}=s_{22}=s_{33}, \\
& s_{12}=s_{13}=s_{23}, \\
& \text { and } \\
& s_{44}=s_{55}=s_{66} .
\end{aligned}
$$

Measured values of the elastic compliances for $\mathrm{NaCl}$ exist in the literature (Nye) ${ }^{(10)}$

$$
\begin{aligned}
& \mathrm{s}_{11}=2.21 \times 10^{-12} \mathrm{~cm}^{2} / \text { dyne } \simeq 2.24 \times 10^{-6} / \mathrm{atm} \\
& \mathrm{s}_{12}=-0.45 \times 10^{-12} \mathrm{~cm}^{2} / \text { dyne }=-0.45 \times 10^{-6} / \mathrm{atm} \\
& \mathrm{s}_{44}=7.83 \times 10^{-12} \mathrm{~cm}^{2} / \text { dyne } \simeq 7.93 \times 10^{-6} / \mathrm{atm}
\end{aligned}
$$

In this work there are two other tensor properties of crystalline material of considerable importance. One is the electric field gradient (EFG) tensor $\phi_{i j}$ which determines the quadrupole interaction, and hence the resonance frequencies. The 2 nd rank EFG tensor is traceless and symmetric (Schempp) $)^{(9)}$, and thus contains five independent componentis, inree ôt which can be thought of as determining the orientation of the principal axes, leaving two independent diagonal elements. In the following we shall adopt the usage that $\phi_{i j}$ represents the general EFG tensor with elements $\partial^{2} / \partial x_{i} \partial x_{j}\left(1 / r^{2}\right)$ having dimensions of $\mathrm{cm}^{-3}$. The parameters of importance to NQR, $q$ and $n$ are related to the components of the tensor $v_{i i}$ in the coordinate system in which $\Phi_{i j}$ is diagonal, where 


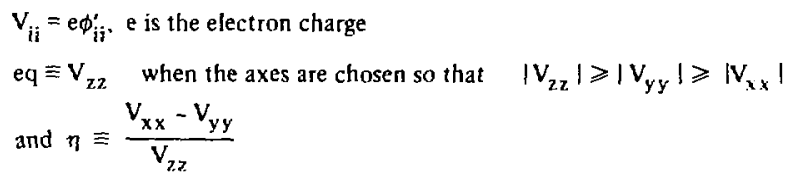

eq therefore has dimensions of volts $/ \mathrm{cm}^{3}$, and can be expressed in terms of frequency by multiplying by $\mathrm{eQ} / \mathrm{h} ; \eta$ is dimensionless satisfying $0 \leq n \leq 1$.

The other tensor property of interest is the "gradient elastic tensor," $g_{i j k l}$ which relates the strain to the EFG via

$$
\phi_{\mathrm{ij}}-\phi_{\mathrm{jj}}^{\mathrm{o}}=\mathrm{g}_{\mathrm{ijk} \mathrm{i}} \epsilon_{\mathrm{k} 1}
$$

where $\phi_{i j}$ is the value of the EFG in the absence of strain. For cubic site symetry, $\phi_{i j}^{0}=0$, giving in matric notation

$$
\phi_{\mathrm{ij}}=\mathrm{g}_{\mathrm{ij}} \epsilon_{\mathrm{j}}
$$

Since the $g_{i j}$ 's are a crystal property, they must obey the same invariance under symetry operations as the elastic coefficients, resulting in a reduction of the 36 elements again to just three in the cubic case, $g_{11}, g_{12}$, and $g_{44}$. However, owing to the tracelessness of the $\phi_{j}$ tensor/matrix, one can only observe $\left(g_{11}-g_{12}\right)$ and $g_{44}$ as can easily be seen by writing out the components

$$
\begin{aligned}
& \Sigma \phi_{i}=0 \text { requires } g_{11}\left(\epsilon_{1}+\epsilon_{2}+\epsilon_{3}\right)+2 g_{12}\left(\epsilon_{1}+\epsilon_{2}+\epsilon_{3}\right)=0 \\
& \text { or } g_{12}=-1 / 2 g_{11}
\end{aligned}
$$


The EFG can be written in terms of the stress by combining equations (2) and (6) or (3) and (7) to give

$$
\phi_{i j}=g_{i, k l} s_{k \mid m n} o_{1 m n}=C_{i m m n} o_{m n}
$$

where

$$
C_{i j m n}=g_{i j k} s_{k} \mid m+n \text {. }
$$

The C's may be called the "gradient-stress" tensor (Shulman). (11) In matrix notation, which will be used uniformly throughout the following, we have

$$
\phi_{i}=g_{i j} s_{j k} o_{k}=C_{i j} o_{j} . \quad i=110 s
$$

Making use of (4) and (8) for the cubic case, one obtains

$$
\begin{aligned}
& \phi_{1}=g_{11} \mid\left(s_{11}-s_{12}\right)\left(\sigma_{1}-1 / 2\left(\sigma_{2}+\sigma_{3}\right) \mid=C_{11}\left[\sigma_{1}-1 / 2\left(\sigma_{2}+o_{3} \mid\right.\right.\right. \\
& \phi_{2} \text { and } \phi_{3} \text { ohtained by cylic permutation of the indices } \\
& \phi_{4}=g_{44} s_{44} \sigma_{4}=C_{44} \sigma_{4} \\
& \phi_{5}=C_{44} \sigma_{5} \\
& \phi_{6}=C_{44} \sigma_{6}
\end{aligned}
$$

Values of the $g$ 's and C's have been measured experimentally by several methods and although there is some scatter in the data, approximate values can be used to determine the magnitudes to be expected in $\mathrm{NaCl}$ (Table 2) (Kanert and Mehring). (12) 
Table 2

Values of the Gradient Elastic and Gradient Stress Tensors in $\mathrm{NaCl}$

$\begin{array}{llccc} & \begin{array}{l}9_{11} \\ 10^{15} \text { dyne }^{\frac{1}{2}} \mathrm{~cm}^{-2}\end{array} & \mathrm{C}_{11} & \mathrm{C}_{44} \\ \mathrm{Na}^{23} & 2.7 & 0.95 & 10^{3} \text { dyne }^{-\frac{1}{2}} & \\ \mathrm{Cr}^{35} & 2.9 & 3.9 & 8.0 & 30.8\end{array}$

All these results have been obtained for compressive stresses and apparently no measurements have been made to date for tensile stresses.

It is possible to calculate the field gradients and NQR frequencies in a few special cases to see the general effects. For this we consider a cubic crystal oriented such that the $[100]$ direction is along $x_{1},[010]$ along $x_{2}$, etc.

1. The simplest case involves a homogeneous uniaxial stress $\sigma_{0}$ applied along [100]

$$
a=o_{0}\left(\begin{array}{lll}
1 & 0 & 0 \\
0 & 0 & 0 \\
0 & 0 & 0
\end{array}\right)
$$


Then

$$
\phi_{1}=C_{11} \sigma_{11} \cdot \phi_{2}=\phi_{3}=-1,2 C_{11} \sigma_{01}=-1,2 \phi_{1} \text {. and } \phi_{4}=\phi_{5}=\phi_{6}=0
$$

or

$$
|0|=-1 / 2 C_{11} \sigma_{n}\left(\begin{array}{rrr}
2 & 0 & 0 \\
0 & -1 & 0 \\
0 & 0 & -1
\end{array}\right)
$$

The maximum component of the EFG is thus parallel to the stress direction, [100], giving $q=e_{\phi_{1}}$ or $e^{2} q Q_{C 1}=46 \mathrm{~Hz} / \mathrm{atm}, n=0$

$$
\mathrm{e}^{2} \mathrm{qQ}_{\mathrm{Na}}=55 \mathrm{~Hz} / \mathrm{atm}, \mathrm{n}=0
$$

Since the NQR frequencies are given for the $I=3 / 2$ case by

$$
v=\frac{1}{2} c^{2} c_{Q} Q\left(1+\frac{\eta^{2}}{3}\right)^{1 / 2}
$$

we obtain in a uniform stress field of $100 \mathrm{~atm}$

$$
\begin{aligned}
& v_{\mathrm{Cl}}=2.3 \mathrm{kH} / \\
& v_{\mathrm{Na}}=2.8 \mathrm{kHz}
\end{aligned}
$$


These results can only be considered accurate to within a factor of 2 . Marsh and Casabella, (8) for example, report equal frequency shifts for the $\mathrm{Na}$ and $i \mathrm{l}$ for [100] stress. Accurate determination of the constants during the detailed laboratory phase of the work is a prerequisite for field work.

2. For a stress applied along [110], the stress tensor becomes, after carrying out a rotation of the coordinates,

$$
\sigma=\sigma_{0}\left(\begin{array}{ccc}
1 / 2 & -1 / 2 & 0 \\
-1 / 2 & 1 / 2 & 0 \\
0 & 0 & 0
\end{array}\right)
$$

Thus

$$
|\phi|=1 / 4 a_{0} C_{11}\left(\begin{array}{ccc}
1 / 4 C_{11} & -1 / 2 C_{44} & 0 \\
-1 / 2 C_{44} & 1 / 4 C_{11} & 0 \\
0 & 0 & -1 / 2 C_{11}
\end{array}\right)
$$

Introducing the quantities $c=s_{44} /\left(s_{11}-s_{12}\right)$ and $\gamma=g_{44} / g_{11}$, $\phi$ can be rewritten as

$$
|\phi|=1 / 4 \sigma_{0} c_{11}\left(\begin{array}{ccc}
1 & -2 \gamma c & 0 \\
-2 \gamma c & 1 & 0 \\
0 & 0 & 0
\end{array}\right)
$$

This must be diagonalized in order to find the NQR parameters, resulting in

$$
10 i=1 / 4 C_{11} \sigma_{11}\left(\begin{array}{ccc}
1+2 \gamma c & 0 \\
0 & 1-2 \gamma c & \\
0 & -2
\end{array}\right)
$$


In $\mathrm{NaCl}$, using the data in Table 2, we find $\mathrm{c}=2.94, \gamma_{\mathrm{Cl}}=1.34$, and $\gamma_{\mathrm{Na}}=$ 0.35. The component of $\phi$ which determines the $z$-axis and $q$ depends on whether $1+2 \gamma c<2$, which means that $q=\phi_{z z}$, or whether $1+2 \gamma c>2$ which means that $q=\phi_{x x} \cdot$ For $C 1,2 \gamma c \simeq 7.88$, giving

$$
\begin{aligned}
& \left.|V|_{(1)}=110 \mathrm{kH}\right)\left(\begin{array}{ccc}
-2 & & 0 \\
& -6.88 & \\
0 & & 8.48
\end{array}\right) \\
& \mathrm{e}^{2}{ }_{\mathrm{qQ}} \mathrm{C}_{\mathrm{Cl}}=10.3 \mathrm{~Hz} / \mathrm{atm}, \eta=0.55 \\
& v_{C 1}=5.2 \mathrm{kH} \% \text { at } 100 \mathrm{~atm}
\end{aligned}
$$

For $\mathrm{Na}, 2 \gamma \mathrm{C} \simeq 2.06$

$$
\begin{aligned}
& |V|_{\mathrm{Na}_{\mathrm{a}}}=1.37 \mathrm{kH} \%\left(\begin{array}{ccc}
-1.07 & & 0 \\
& -2 & \\
0 & & 3.07
\end{array}\right) \\
& \mathrm{e}^{2} \mathrm{qQ}_{\mathrm{N}_{\mathrm{a}}}=42.2 \mathrm{H} \mathrm{\textrm {m }} / \mathrm{atm} . \eta=0.02 \\
& \nu_{\mathrm{Na}}=2.1 \mathrm{kH} \text { z at } 100 \mathrm{~atm}
\end{aligned}
$$

For stress applied along [110], the principal axes directions are along [001], [110], and $[\overline{1} 10]$.

3. If the stress is applied along the [111] direction, we first carry out a rotation of $45^{\circ}$ around the z-axis, and then about $y^{\prime}$-axis by $35.26^{\circ}$

$$
\begin{gathered}
a^{* \prime}=\mathrm{BAOA}^{-1} \mathrm{~B}^{-1} \\
\sigma_{111}=1 / 3 \sigma_{0}\left(\begin{array}{lll}
1 & 1 & 1 \\
1 & 1 & 1 \\
1 & 1 & 1
\end{array}\right) \\
101=1 / 3 \mathrm{C}_{44} a_{0}\left(\begin{array}{lll}
0 & 1 & 1 \\
1 & 0 & 1 \\
1 & 1 & 0
\end{array}\right)
\end{gathered}
$$


which after diagonalizing, yields

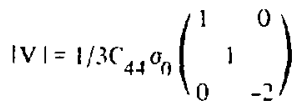

$$
\begin{aligned}
& \therefore{ }^{2} \mathrm{BO}_{(1)}=119 \mathrm{H} / \mathrm{arm}, n=0 \\
& u_{11}=6.0 \mathrm{k} 1 \mathrm{kz} \text { at } 100 \mathrm{~atm} \\
& \mathrm{e}^{2} \mathrm{q} \mathrm{Q}_{\mathrm{Na}}=36 \mathrm{H} / \mathrm{i} / \mathrm{alm}, \eta=0 \\
& { }_{1 \mathrm{x}: \mathrm{a}}=1.8 \mathrm{kHL}: \mathrm{at} 100 \mathrm{~atm}
\end{aligned}
$$

where the $z$-axis is along $[111]$.

The intensity of the NQR lines depends upon the orientation of the linearly-polarized rf exciting field to the axis of the cylindrically symmetric EFG (the $n \neq 0$ case is more complicated). The intensity of the lines is a maximum when tha field lies in a piane perpendicular to the symmetry axis (the z-direction) and is zero when the field is parallel to the symmetry axis (Lucken). (13) Triis fact must be taken into account when working out the line-shapes to be expected for the general homogeneous stress. For example, in the [110] case, if the rf field is parallel to the stress direction, the line will not be observed.

No more than an estimate of the situation in a randomly-oriented polycrystalline sample can be given at this $t$ ne. To do the calculation properly, one must determine the probability that a crystallite wi?l have a given orientation with respect to the applied stress and weight the ensemble of field gradients by this orientation factor and the transition probabilities. However, it can be seen that the three special r.ases considered above represent extreme cases, and every other orientation must give rise to frequencies lying in the regions shown in Table 3. 


\section{Tabie 3}

NQR Frequencies (in $\mathrm{kHz}$ ) in $\mathrm{NaCl}$ as a Function of Orientation at $\sigma=100 \mathrm{~atm}$

$\begin{array}{llll} & {[100]} & {[110]} & {[111]} \\ \mathrm{Na}^{23} & 1.8 & 2.1 & 2.8 \\ \mathrm{Cl}^{35} & 2.3 & 5.2 & 6.0 \\ \mathrm{Cl}^{37} & 1.8 & 4.1 & 4.7\end{array}$

A completely random distribution, then, will result in lires approximately 3.5 $\mathrm{kHz}$ wide for $\mathrm{Cl}$ and $1 \mathrm{kHz}$ wide for $\mathrm{Na}$. A computer program is being generated to work out the line shapes in detail.

The petrofabric in the region of a salt-dome borehole may not present a wholly random orientation of the crystallites. In certain beds, very large "single" crystals with dimensions or the orjer of a foot or more have been found (Muehlberger). (14) It is generall; accepted that a preferred orientation of crystallites in the final fabric is strongly related to the stress system applied (Kern). (15) There is some evidence that the [001] axes in halite are preferentially riented parallel to the principa' stress direction, i.e. [001) perpendicular to the axial planes of isoclinjl folds in salt domes, which is in agreement with themodynamic theory for stable crystal orientations under nonhydrostatic stress (Carter and Heard ${ }^{(16)}$ and Kamb ${ }^{(17)}$ ). Therefore, although a polycrystalline mass may be expected, the crystallites may display ; large degree of local order, i.e., a narrow distribution of orientation angles may exist. This will result in the NQR spectra also ha:ing narrower widths. 
In the general case of stresses underground, the stress tensor is not uniaxial, but triaxial, i.e.,

$$
\sigma=\left(\begin{array}{lll}
\sigma_{1} & & 0 \\
& \sigma_{2} & \\
0 & & \sigma_{3}
\end{array}\right)
$$

The effect of additional non-zero elements in the stress tensor is generally additive, taking appropriate care of the signs. For a crystal having [001] // $\sigma_{3}$ and $[010] / / \sigma_{2}$

$$
|\phi|=C_{11}\left(\begin{array}{cc}
\sigma_{1}-1 / 2\left(\sigma_{2}+\sigma_{3}\right) & 0 \\
\sigma_{2}-1 / 2\left(\sigma_{1}+\sigma_{3}\right) \\
0 & \sigma_{3}-1 / 2\left(\sigma_{1}+\sigma_{2}\right)
\end{array}\right)
$$

It can be seen that in the case of hydrostatic pressure, $\sigma_{1}=\sigma_{2}=$ $\sigma_{3}$, this result assures that $|\phi|=0$ as must be true in a cubic crystal. If $\sigma_{3}=100 \mathrm{~atm}$, and $\sigma_{1}=\sigma_{2}=50 \mathrm{at}$ il, a situation which might be encountered at depth, we find

$$
\begin{aligned}
& \mathrm{e}^{2} \mathrm{qQ}_{\mathrm{Cl}}=2.3 \mathrm{kHz}, \eta=0 \\
& { }_{\mathrm{Cl}}=1.15 \mathrm{kHz} .
\end{aligned}
$$

Another factor to be considered is that the stress distribution around a borehole in a previously uniform stress field is no longer uniform, but the 
radial component of the stress $\sigma_{r} i$ a function of the distance from the borehole wall. At $\theta=0$, for example,

$$
\sigma_{\mathrm{r}}=a_{1}\left(1-\frac{5}{2} \frac{\mathrm{R}^{2}}{\mathrm{r}^{2}}+\frac{3}{2} \frac{\mathrm{R}^{4}}{\mathrm{r}^{4}}\right)+\sigma_{3}\left(\frac{3}{2} \frac{\mathrm{R}^{2}}{\mathrm{r}^{2}}-\frac{\vdots}{2} \frac{\mathrm{R}^{4}}{\mathrm{r}^{4}}\right)
$$

which means that crystallites will experience differing stresses depending on their distance from the borehole wall (Cook). (2) The effect of such an inhomogeneous stress will be to further broaden the NQR lines. This can be taken into account by snectral line narrowing techniques such as deconvoluting the NQR lineshapes with the appropriate broadening function.

One final factor enters the picture. The frequencies of NQR lines sometimes show a temperature dependence which is related to the thermal motion of the atoms in the crystal lattice. In the case of salt, this effect will make the gradient elastic tensor, $g_{i j}$, temperature dependent. The elastic compliances, $s_{i j}$, will also be expected to show some temperature dependence, but for $T<200^{\circ} \mathrm{C}$ this is expected to be small. Two different values for the geothermal gradient in Gulf Coast salt domes have been measured: $36^{\circ} \mathrm{C} / \mathrm{km}$ and $22^{\circ} \mathrm{C} / \mathrm{km}$ (Nichols ${ }^{(18)}$ and Moses ${ }^{(19)}$ ). Therefore, temperatures $20^{\circ}$ to $60^{\circ} \mathrm{C}$ above ambient may he expected in typical boreholes of interest. No information on the sensitivity of the values for $\mathrm{NaCl}$ in Table 2 to changes in temperatures is available at this time. From other NQR information, however, these variances are not expected to be critical. These would be measured in laboratory studies over the anticipated temperature ranges prior to field trials. 


\section{Resonance Techniques}

Based on the results of Section 8 , the problem is to detect either the sodium or the chlorine quadrupole coupling in sufficient detail to extract the strain/stress information. The quadrupolar coupling will lie in the $1-15 \mathrm{kHz}$ region for stresses up to $300 \mathrm{~atm}$ and the lines will be, perhaps, $1-10 \mathrm{kHz}$ wide, depending on the degree of crystallite misalignment; the nature of the impurities, dislocations, and other crystal imperfections; and the inhomogeneity of the stress field resulting from the borehole deformation. There are three basic approaches: (1) direct detection (pure NQR), (2) quadrupolar perturbed NMR, and (3) double resonance methods. Each of these techniques has numerous variations. These are discussed below.

1) Direct Detection

Direct detection of the proton NMR signals in the earth's magnetic field $(\sim 0.5 G)$ is routinely carried out in magnetometers; the resonant frequency here is ca. $2.1 \mathrm{kHz}$. NQR in chlorine will be more difficult because (a) the sensitivity of $\mathrm{Cl}$ NMR relative to protons is $4.7 \times 10^{-3}$, (b) the magnetometers employ a liquid which has a sharp line in contrast to the broad line from solid $\mathrm{NaCl},(c)$ the proton instrument can be optimized for a single frequency, and (d) line shape information is not required. Overall it is likely that the instrinsic $C l S / N$ ratio will be at least $10^{-5}$ times smaller.

The idea, nonetheless, would be to apply a strong pulse to the sample in such a way that it's Fourier spectrum covers the bandwidth of interest, nominally a $D C$ delta function. This would excite NQR transitions. A tuned receiver would then receive free induction decay signals, and the total spectrum mapped out by changing the receiver center frequency. Standard pulse techniques utilizing $90^{\circ}$ and $180^{\circ}$ pulses probably will not work here 
because at $5 \mathrm{kHz}$ "rf frequency" a single cycle is $0.2 \mathrm{msec}$ long, which will not be short compared to $1 / T_{2}$ as required $\left(T_{2} *\right.$ is the dipolar linewidth).

Such an experiment has not been previously performed to the authors' knowledge, and would involve a considerable theoretical and engineering effort to deternine its practicality. On the positive side, it el iminates the need for a large magnetic field and many complications from attempting remute detection of a double resonance signal, but it would not eliminate the intrinsic over lap between the []$^{35}, \mathrm{Cl}^{37}$, and $\mathrm{Na}^{23}$ spectra.

This overlap arises from the fact that the quadrupole coupling constarts of the three nuclear species present fall in the same range. Although the exact 7 ine shapes are not yet known, on the basis of preliminary considerations each nuclear species may produce a line something like the sketch below in a randomly-oriented polycrystalline sample.
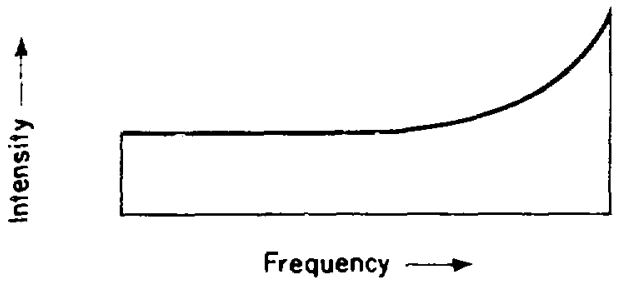

XRL $8012-2481$.

Since the two chlorine isotopes have quadrupole frequencies simply related by the ratio of their quadrupole moments, $Q^{C l-37} / Q^{C !-35}=0.7883$, and intensities related by their isotopic abundance $\left(C 7^{35}=.5 \%, C 7^{37}=25 \%\right)$, there will be a superposition which can be determined. The position of the sodium line does not bear a deterministic relationship to the chlorines because the values of the $\mathrm{Cl}$ and iva gradient-elastic tensors differ, presumably owing to Sterriheimer $(13)$ anti-shielding effects. The composite line 
will thus have roughly the form shown (for a random polycrystalline sample at $100 \mathrm{~atm}$ ) in Figure 1.

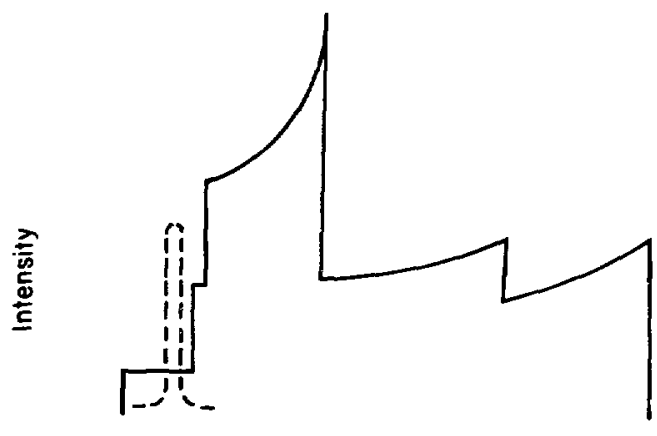

Frequency

XBL $8012-2488$

Figure 1. Shape of a polycrystalline NQR line in stressed $\mathrm{NaCl}$ (estimated).

The dotted line at $2.1 \mathrm{kHz}$ will arise from the hydrogen in water resonant in the earth's magnetic field. This asymetric line shape may prove to be an aid in identifying and measuring the spectral parameters related to stress.

Estimates of the water content in interstices and inclusions range from 0.1 to $1 \%$. Although this percentage is low, the proton signals are relatively strong, and thus it is likely that the proton liquid NMR signal will appear. It will, however, be a constant independent of orientation and depth and, therefore, probably can be subtracted out. (The Na NMR frequency in the earth's field is $550 \mathrm{~Hz}$, and that for the chlorines is even lower.)

2) Quadrupolar Perturbed NMR

In an NMR experiment a large, reasonably homogeneous magnetic field would have to be provided. For this discussion we will assume that a $1 \mathrm{kG}$ field can 
be obtained. In this case the chlorines will have a Larmor frequency of 0.4 $\mathrm{MHz}$, and the sodiums will have a frequency of $1.12 \mathrm{MHz}$. This effects almost complete separation of the spectra, eliminating overlap. The sodium quadrupolar interaction is in the range of $2+1 \mathrm{kHz}$. This method has been used to measure the quadrupole frequencies of neighboring shells around $\mathrm{Br}$ impurities in doped $\mathrm{NaCl}$ (Andersson ${ }^{(20)}$ ). It is weil known that, when the quadrupole interaction is small compared to the Zeeman interaction, the transition energies are shifted, giving rise to a "powder pattern" in a random polycrystalline sample. (21)

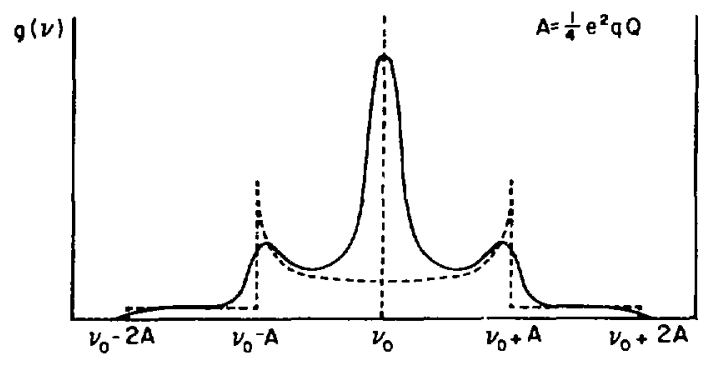

Figure 2. First-order quadrupole perturbation. Line shape for $I=3 / 2$ in powdered samples of axially symmetric crystals (broken line). With dipolar broadening superimposed the full curve results. Frequently the satellites are spread out over such a large frequency range. that the wings become unobservable.

In the event that there is a range of quadrupole interactions in the solid, a superposition of powder patterns is observed, which tends to produce a broad line. In this case, the details of the quadrupole interactions will have to be extracted in a differer: way, by using computer fitting, instead of visual inspection. 


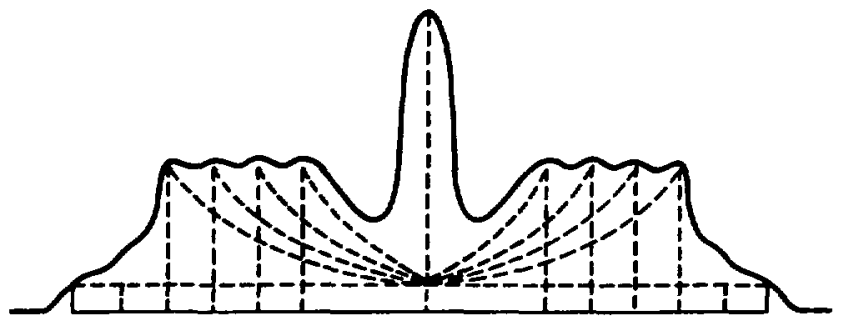

XBL $8012-6545$

Figure 3. The overlap of many spectral components results in a broad line spectrum.

Since the interaction remains small enough that first-order perturbation theory is applicable, an unshifted "central" transition remains from the $+\frac{1}{2} \rightarrow-\frac{1}{2}$ spin states (Cohen and Reif). (21) This central transition contains no direct information about the quadrupole interactions present, but its anplitude grows smaller as more and more intensity is shifted to the wings. This feature has been used to measure quadrupole effects, including those due to stresses in cubic materials (Kanert and Mehring) ${ }^{(12)}$ when a single value of $e^{2} q Q$ is present throughout the sample. This approach will be complex to use in the presence of an inhomogeneous EFG such as expected in natural systems.

If a sufficient number of resolution elements can be obtained, the NMR method offers experimental simplicity. A magnetic field can be generated by a solenoid coaxial with the borehole, a simpie $90^{\circ} \mathrm{rf}$ pulse applied, and the free induction decay (FID) recorded. The FID contains all the line-shape information (Lowe and Norberg). (22) Corrections for magnetic field and $r f$ field inhomogeneities would be required. 


\section{3) Double Resonance}

Double resonance techniques have been developed in recent years which offer enormous increases in sensitivity in the detection of weak NMR and NQR signals. The idea behind double resonance techniques in magnetic resonance is to use the strong signal presented by one nuclear species (A) to detect a weak signal offered by another species $(R)$, where the weakness may arise from low isotopic abundance, low frequency, or long relaxation time. In the case of quadrupole effects in stressed $\mathrm{NaCl}$, the problem is the low frequencies of the stress-induced quadrupole coupling constant ( $Q C C)$. A number of different schemes have been developed to obtain a gain in sensitivity.

These techniques consist essentially of "NQR calorimetry" performed between two spin systems which can be prepared at different spin temperatures. The $A$ spins are abundant and this magnetization is easy to measure. The second species $\mathrm{R}$ are rare or otherwise difficult to detect directly. The experimental cycle consists of a basic four-step process:

i. The A spins are prepared in a polarized state;

ii. The $R$ spins are polarized by making thermal contact between the two spin systems;

iii. An oscillating magnetic field of frequency $w_{R}$ is applied to the sample at the R-spin transition frequency;

iv. Steps $i i$ and $i j i$ are repeated several times, or maintained for a long time, and the state of the $A$ spin system is re-determined. A heating of the $A$ spins is evidence for power having been absorbed by the $R$ spins, and thus the $R$ resonance is detected indirectly.

Of several double resonance methods, a few can be dismissed as not suitable for the experiments on salt. These include spin echo double resonance (SEDOR) because large static magnetic fields are required giving no advantage 
over conventional resonance methods; double resonance in the rotating frame (DRRF) suffers from the same defect; double resonance in the laboratory frame (DRLF) is not generally suitable for detection of quadrupole interactions below $200 \mathrm{kHz}$, and in any case often renders details of the line shapes unobservable.

This leaves two methods with a high probablity of success--spin-mixing by level crossing (DRLC) and continuous coupling. Both methods are closely related (Koo ${ }^{(23)}$, Hahn ${ }^{(24)}$, Edmonds $\left.{ }^{(25)}\right)$. Continuous coupling requires magnetic fieid cycling and a three-step experimental cycle: polarization, irradiation, and detection. The sample is intially placed in a large magnetic field $H_{0}$ for a time sufficient for both sets of spins (the $A$ and the $R$ ) to reach equilibrium with the lattic temperature $T_{L}$. This time will be at most on the order of several times the longest $T_{1}$ in the sample; since $T_{1}(\mathrm{Na})=12$ secs, the equilibrium time might be as much as 30 secs. The magnetic field should be as large as practicable, about $1 \mathrm{kG}$ for a field instrument or $10 \mathrm{kG}$ for a laboratory set-up. A $1 \mathrm{kG}$ field is practical in the presently conceived in-situ measuring configuration.

The sample is now removed bodily from the magnetic field, or the field is switched rigorously off in a time as short as possible, $<1 \mathrm{sec}$. During this time, the Zeeman splitting of the A spins ( $\left.\mathrm{Na}^{23}\right)$ collapses, and in doing so crosses over the quadrupole energy levels of the $\mathrm{R}$ spins $\left(\mathrm{C} 1^{35}\right.$ and $\left.\mathrm{Cl}^{37}\right)$. The $R$ spins thus become partially polarized by energy exchange, and now reside in a zero applied field.

Immediately after the $H_{0}$ field is zero, a continuous $r f$ field at frequency $\omega_{R}$ (the chlorine transition frequencies) is applied for a time 
necessary to saturate the $R$ spins (3-5) secs). At the completion of this irradiation, the magnetic field $H_{0}$ is switched back on again, and the $A$ spin magnetization measured, by applying a single $\pi / 2$ pulse and observing the free induction decay signal (FID). This cycle is repeated for different values of $\omega_{R}$ until the region of interest is covered--in the case of $c 1$ in salt, 2-10 $\mathrm{kHz}$. Before the start of each new cycle, a saturation comb of $n / 2$ pulses for the A spins initializes the polarization in that system.

The sensitivity of the method depends on the number of $R$ system "spin flips" which occur during the R-irradiation phase. This will depend upon the number of $\mathrm{Cl}$ transitions excited within the bandwidth of the $\omega_{R}$ pulse when it is desired to detect a broad line, as in the case of salt. The sensitivity can be very high, particularly for the spin $3 / 2$ case (Edmonds). (25) It is not clear how much resolution may be obtained. Double resonance by continuous coupling has had consideratle success in detecting QCC's in deuterium, ${ }^{2} H$, at natural abundance (Edmonds and Mailer), (26) and this problem presents many of the same features found in the sait problem. It therefore appears that sufficient resolution is available.

The experimental procedure in this case will present a number of challenging problems, one of which is that all the species present will 
possess multiple energy levels which will collapse and cross over when the field is switched off in a complex way (Figure 4).

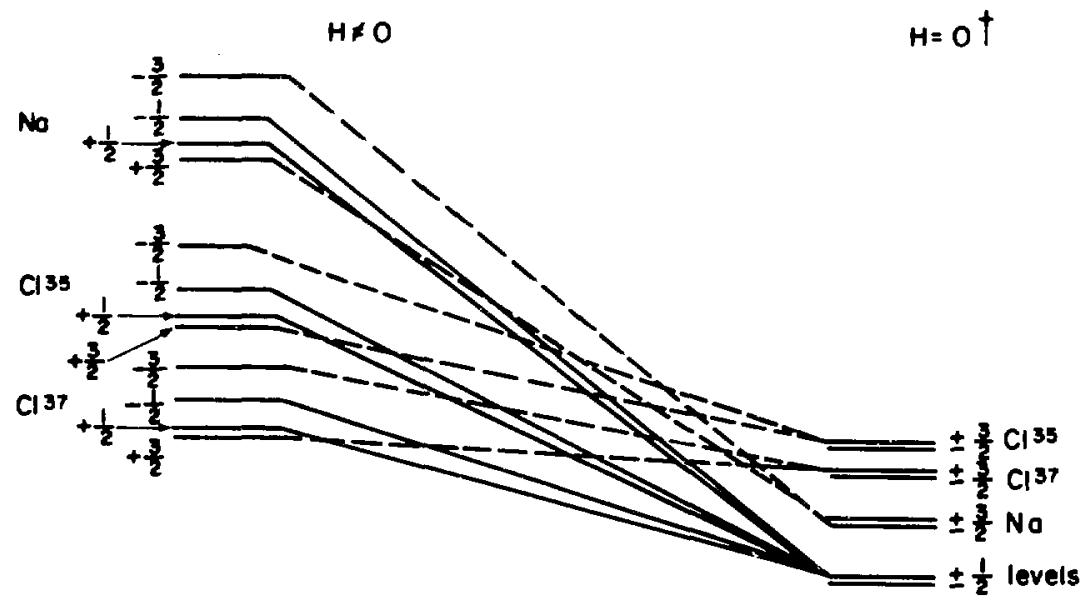

$X B ! 8012-2476$

Figure 4. NaCl level crossing diagram.

'The earth's field is always present, of course, producing splittings on the order of a few hundred hertz in the pure quadrupole frequencies.

Although these multiple crossings as the energy manifolds collapse present considerable theoretical difficulties to analyze in detail, it is not obvious that it makes the experiment any more difficult nor the extraction of the information need to determine stress. The "central" $+\frac{1}{2} \rightarrow-\frac{1}{2}$ transition in the sodium spectrum can be monitored as the "abundant" species to detect the low quadrupole frequencies in the chlorines. Because of the overlap, the observed spectrum will be a superposition of three lines as shown in figure 1. 
The continuous coupling procedure differs from a level crossing experiment (DRLC) only subtly. In the latter, a partial polarization in the $R$ spin magnetization is introduced as the spin multiplets collapse to their zero-field values. If during the irradiation phase up corresponds to an allowed $R$ spin transition frequency, power is absorbed, changing the $R$ spin level populations. When the $H>0$ state is restored, additional level crossings take place allowing a trarisfer of energy ("heat") between the $R$ and A spins.

The so-called zero-field energy levels are not in fact in a strictly $H=0$ state. For one reason, the earth's magnetic field is still present, which gives energy levels of a few hundred hertz. More important is the dipolar coupling between the spins, which may produce effective internal fields of several gauss, and thus produce energy levels extending up to tens of kilohertz. (In fact, it is this internal dipolar coupling which makes energy transfer between the spins possible and thus is essential to the whole experiment.) Thus, at very low values of the coupling constant, the two spin systems make continuous thermal contact in zero applied field owing to partial overlap of their absorption spectra. Irradiation at $w_{k}$ then also causes transitions among the A spin levels. Hence, there is a competition at a given frequency between direct absorption by the $A$ spins and absorption by the $R$ spins and subsequent transfer of power to the A spins by mutual spin flips which conserve the total spin energy. The relative probability of these two absorption mechanisms depends on many factore e.g. the density of A spin states at the irradiation frequency compared with that of the $R$ spins, the relative magnitudes of the matrix elements which govern photon absorption, and spin diffusion effects. The competition between direct absorption by the $A$ 
spins and indirect absorption through the $R$ spins has been shown not to be a problem. For example, in the case of $5 \%$ enriched $\mathrm{D}_{2} \mathrm{O}$ in $\mathrm{H}_{2} \mathrm{O}$, it was possible to destroy virtually all the proton magnetization by irradiating the deuteron quadrupole resonance with such a low power level that almost no proton absorption occurred. The ultimate limitation on the sensitivity, therefore, is the competition offered by direct $A$ spin absorption (Edmonds).

This can only be answered by laboratory experiments.

The foregoing discussion suggests that the two most promising approaches are quadrupolar perturbed NMR and double resonance by continuous coupling (DRCC). Both schemes present some difficulties, but they do seem surmountable using existing technologies. These incrude the following: (a) A magnetic field produced by a coil generates heac owing to ohmic losses. A cooling system capable of dissipating a few kilowatts will, therefore, have to be provided. (b) For DRCC, it will be necessary to turn the magnetic field off and on in short times, less than about $1 \mathrm{sec}$, and this requires careful but realizable engineering design. (c) Any magnetic field generated in a borehole will fall off in intensity with penetration depth and thus only the nuclei in an annulus, not more than $1 \mathrm{~cm}$ wide, can be effectively in the same field. The resulting field inhomogeneity makes a more complex NMR signal or FID, and decreases the linewidths of the FID and echo, thus requiring fast electronics. (d) The field inhomogeneity limits the amount of material which can be sampled at any one time. This can be partially compensated for by making an ovalshaped rf coit, perhaps $1 / 10$ of the borehole circumference as its smail diameter and up to about $150 \mathrm{~cm}$ lang. This would encompass a voline of several liters ( $>5 \mathrm{kq}$ of salt). 
(e) The last problem is the intrinsic overlap of the $\mathrm{Na}^{23}, \mathrm{Cl}^{35}$, and $\mathrm{Cl}^{37}$ (and possibly $\mathrm{H}^{l}$ ) signals in tire spectrum, if DRCC is used. This factor, plus the fact that the lineshapes w/l depend on the stress field, the orientation of the crystals, and the orientation of the rf exciting field to both of the foregoing, means that it is unlikely that there will be any simpie one-to-one correspondence between visually recognizable features in the spectrum and the stresses. However, mathematical treatments of the spectra, e.g. Fourier transforms, (27) together with a sufficient laboratory data base, make it possible to process the input information in the computer and output a value directly related to stress. Thus field measurements could be related to the stress by reference to model suectra obtained from careful laboratory measurements. This phase of the work must be begun before detailed elaboration on this point can be given. There will also be contributions to the NQR spectra from lattice defects which must be studied.

Based on extent knowledge the LBL and LLNL technical staffs believe the DRCC method has a high chance of success. As a back up, the NMR perturbetion method can be considered. Most of the equipment items will remain the same between these two system technologies, so the cost of switching from one to the other will be minimal.

\section{CHOICE OF MEASUREMENT TECHNIQUE}

Several major choices have been mide $i$, designing and predicting the potential of a borehole NQR stress/strain sensor.

\section{1) Choice of Measurement Technique}

The pure NQR stress-induced absorption in NQR occurs at a few $\mathrm{kHz}$ frequencies, where transitions saturate at extremely low power levels, 1/f 
noise becones a severe problen, the sensitivity of electronics and receiver coils is much reduced, and spatial and directional resolution is limited.

By going to the double resonance method as discussed in Section [1.C.3, the NQR excitation is measured as a perturbation of an NMR signal observed at a higher frequency in the presence of a strong magnetic field. The inconvenience of having to furnish this field is far less than the difficulties associated with solving the above problems. On the other hand, field nonuniformity restricts the effective sample volume more the higher the center frequency, for a given bandwidth. After detailed calculation, howeve, the $S / N$ levels for pure NQR measured directly and via the dcuble resonance are 37 and 320 , respectively. This represents a substantial gain for the double resonance technique even while disregarding its engineering virtues.

Occasions have been reported in the literature where the $S / N$ gain of the double resonance is not realized because energy transfer does not occur rapidly enough, and the various magnetizations relax to thermal energy before being measured. Here we must remember that these energy transfer rates show order of magnitude increases when the NQR and NMR energies are exactly equal (resonance) as will happen at some point as the field drops, since NMR energies are field dependent and NQR ones are not. However, since the field spends equal time at all intermediate values, it must either spend too much time in the resonance region or too much in the entire process of reducing the field. Either way, sensitivity losses occur. In the present situation, however, we know exactly at what field level the resonance is expected, because for a particular NQR irradiation frequency there is a specific matchirg magnetic field. We thus can irradiate at a specific NQR frequency and follow this by a double resonance measurement in which the falling field 
drops rapidly to the resonance levels, waits there an optimum time, and then completes its drop.

\section{2) Choice of Mapping Method}

Because the principzl stress contours perpendicular to the direction of the cyijndrical hole produce a dipolar field, it is theoretically possible to map this field by measuring the strain distribution and calculating the direction and magnitude of the stress causing the observed strain.

Two methods can be used for measuring stress/strain in salt formations. The first one generates a three-dimensional map of NQR transition frequencies (and thus stress/strain) in the strata around the sample, and carries this map far enough from the borehole to get out of the region of stress/strain disturbances produced by the borehole. The other measures the integral signal at each azimuth from the surface layers of the borehole, and measures the azimuthal variation produced by the barehole stress/strain disturbance. The measured disturbance is linked to the original stress/strain by a functional relationship that is then inverted.

3) Mapping the Surrounding Strata by NQR

The spectrometer will be tuned to the specific frequency of the $\mathrm{Na}^{23}$ NMR line at the magnetic fielo' used. The measurement technigue will be spin echo NMR, in which the sample gives a delayed "echo" pulse a few milliseconds after irradiation with a radio pulse, for which the field-intensity pulseduration product has a specific value. Since the radiofrequency magnetic field falls away rapidly with distance from the transmitting coil, only points along a specific ridial distance element around the borehole axis will contribute to the signal for a given power in the coil. By changing the power 
in the transinitting coil, this radial distance will change, and maps of the NQR signal as a function of radius can be made by scanning the coil power (Figure 5).

A similar technique can be used to discriminate the radial direction. By using appropriate coil shapes (supplemented if necessary by ferrite inserts) the field it produces can be made completely circularly asymetric, so that the proper field is reached at different ranges in different directions. Three possible configurations are; a "horseshoe", Figure 6, a canted transmitter loop with receive loops above and below, Figure 7 , and an array of three or more half wave dipoles, Figure 8.

The "horseshoe" provides some control of close-in fields with and without high permeability cores, but may not provide any significant directivity even at a small number of multiples of the pole spacing. Figure 9 is an heuristic illustration of the magnetic field for this kind of configuration. The length $I$ is long compared to the diameter d. Magnetic field strength vs. distance and angle, centered on the length in a plane perpendicular to the faces, can be calculated. If this directional transmitting coil is now conbined with a rotating, directionally-sensitive receiver coil, a map of NQR signals as function of azimuth and radius vector can be constructed.

The vertical scan can now be provided by simply moving the coils and their associated electronics up and down. 


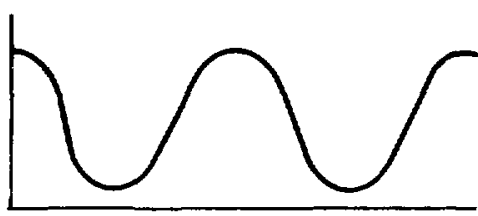

Medium field

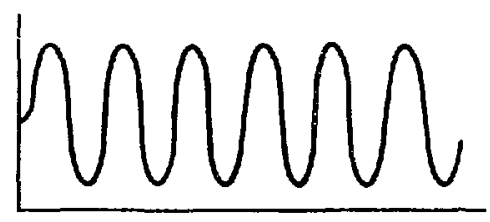

High field

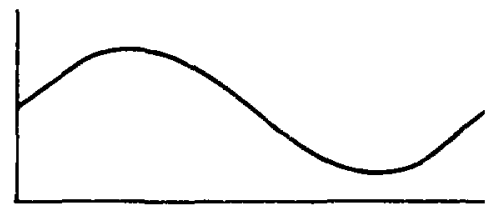

Low field

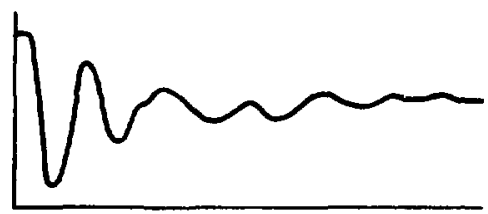

Sum output

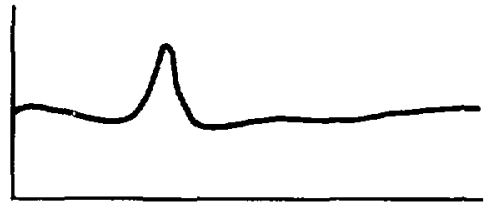

FFT: Intensity vs Ronge

XBL 8012-2485 
$-40$

FIGURE 6

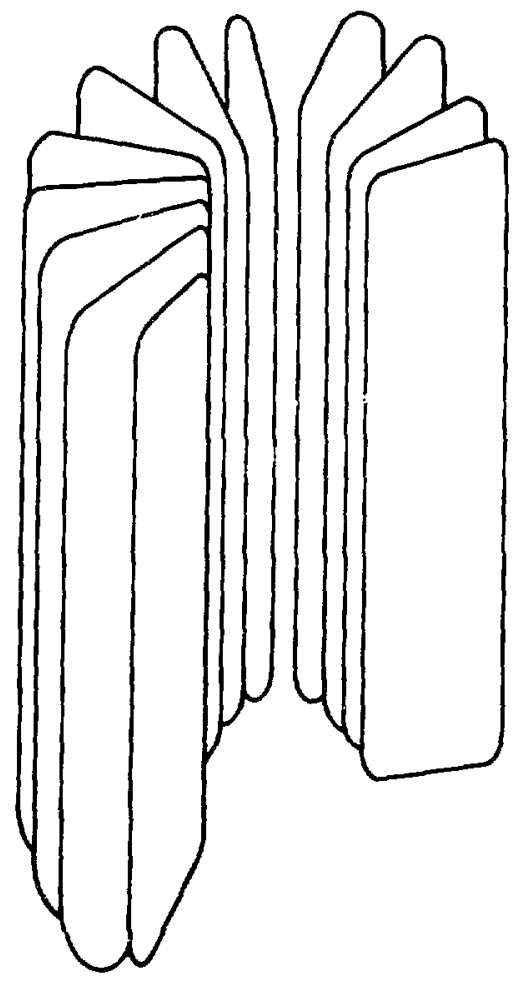

XBL $8012-2480$

FIGLRE 6. HORSESHOE COIL FGR IIRECTIONAL TRANSMI TTER 


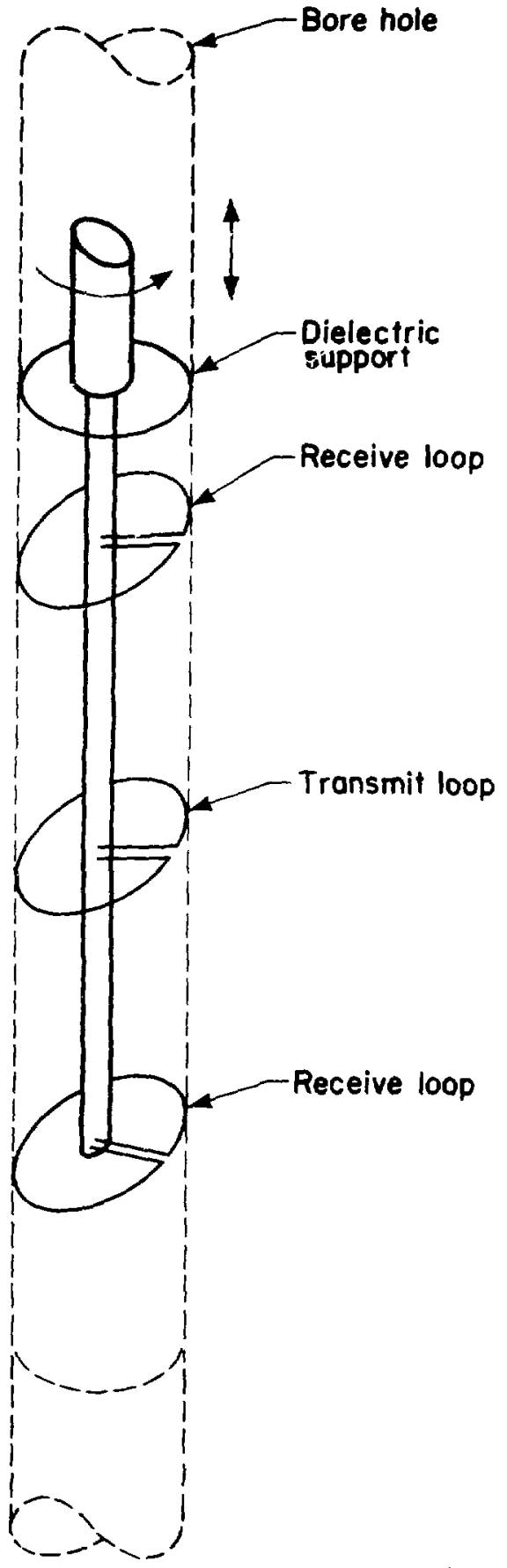

XBL $8012-2486$

FIGURE 7. CANTED TRANSMITTER/RECEIVER LOOPS FOR SPATIAL RESOLUTION 

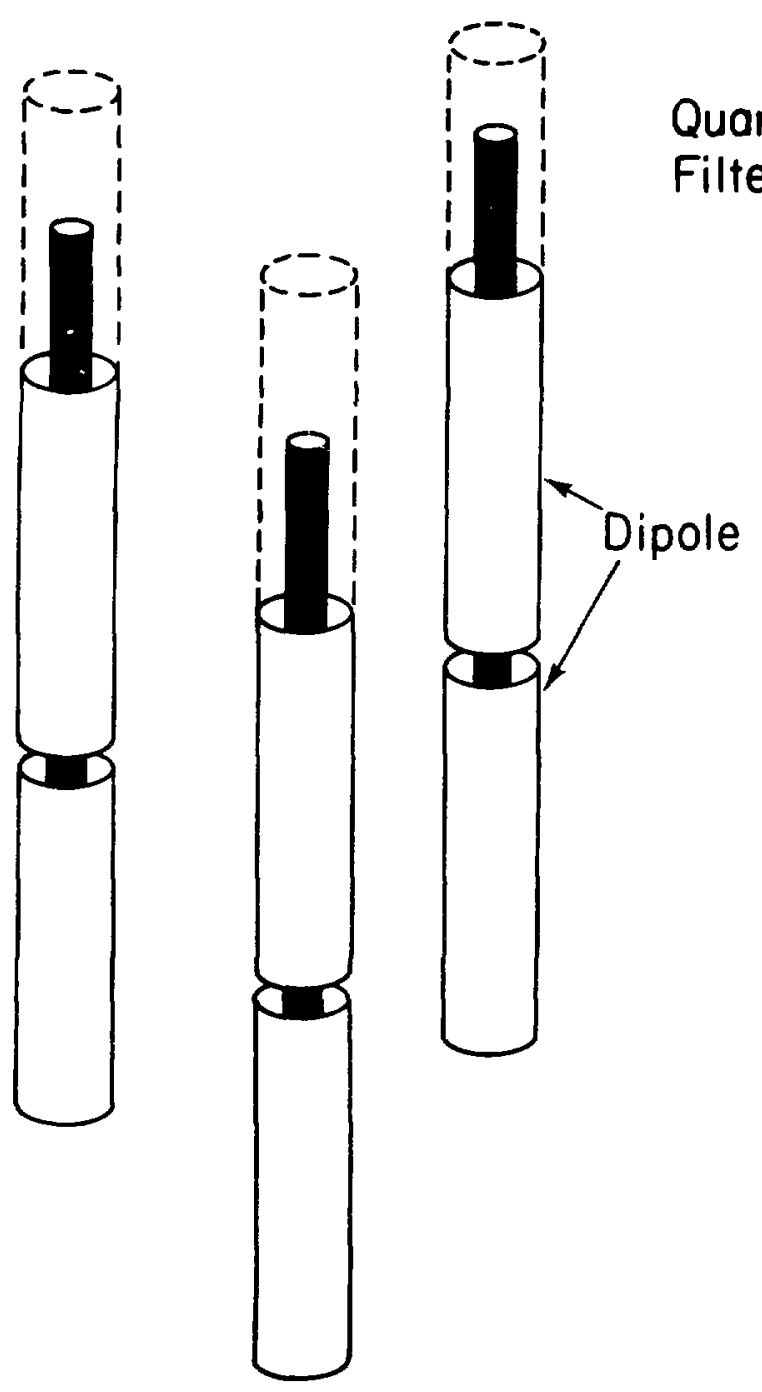

Feed 


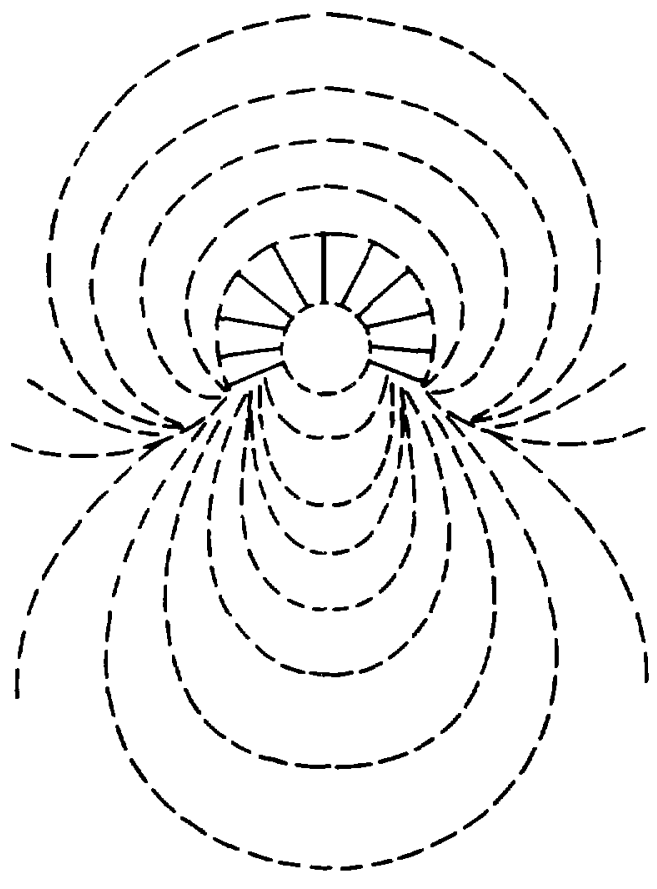

XBL 8012-2479

FIGURE 9. MAGNETIC FIELD LINES OF HORSESHOE COIL 
4) Mapping the Borehole Perturbation by NQR

To use the borehole perturbation approach, the details of the borehole stress/strain field interaction must be considered. The stress tensor can be revolved into three orthogonal principal stresses, $\sigma_{1}$, $\sigma_{2}$ and $\sigma_{3}$, the planes containing any two being those of zero shear stress, and the three principal shear stresses, $\Sigma_{1}, \Sigma_{2}$, and $\Sigma_{3}$.

It is normally assumed that one of the principal stresses acts vertically and is due to 1 thostatic pressure, and that the remaining two are oriented horizontally. This is not always the case but for the purpose of this discussion, is first assumed to be so. A more general case, in which this orientation does not hold, can be taken into account quite readily. According to Cook, (2) "many mine excavations such as boreholes, shafts, and drifts can be approximated closely by a cylindrical hole. The most useful single analytical solution in rock mechanics is, therefore, that for the region outside a circular hole with given principal stresses at infinity. If the principle stresses at infinity normal to the axis of the hole are $\sigma_{v}$ and $\sigma_{h}$ and the radius of the hole is $R$, the siate of stress outside the hole is given by

$$
\begin{aligned}
& \sigma_{\mathrm{r}}=\frac{1}{2}\left(\sigma_{\mathrm{v}}+\sigma_{\mathrm{h}}\right)\left(1-\frac{\mathrm{R}^{2}}{\mathrm{r}^{2}}\right)+\left(\sigma_{\mathrm{v}}-\sigma_{\mathrm{h}}\right)\left(1-\frac{4 \mathrm{R}^{2}}{\mathrm{r}^{2}}+\frac{3 \mathrm{R}^{4}}{\mathrm{r}^{4}}\right) \cos 2 \theta \\
& \sigma_{\theta}=\frac{1}{2}\left(\sigma_{\mathrm{v}}+\sigma_{\mathrm{h}}\right)\left(1+\frac{\mathrm{R}^{2}}{\mathrm{r}^{2}}\right)-\frac{1}{2}\left(\sigma_{,}-\sigma_{\mathrm{h}}\right)\left(1+\frac{3 \mathrm{R}^{4}}{\mathrm{r}^{4}}\right) \cos 2 \theta \\
& \sigma_{\mathrm{r} \theta}=\frac{1}{2}\left(\sigma_{\mathrm{v}}-\sigma_{\mathrm{h}}\right)\left(1+\frac{2 \mathrm{R}^{2}}{\mathrm{r}^{2}}-\frac{3 \mathrm{R}^{4}}{4}\right) \sin 2 \theta,
\end{aligned}
$$

for any radius, $r$, and direction, $\theta$, measured counterclockwise from the vertical. 
"Equations (30-32) above define the total state of stress. The change from the original state of stress induced by excavating the hole is given by the following components of these equations.

$$
\begin{aligned}
& \sigma_{\mathrm{r}}{ }^{\prime}=\frac{1}{2}\left(\sigma_{\mathrm{v}}+\sigma_{\mathrm{h}}\right)\left(-\frac{\mathrm{R}^{2}}{\mathrm{r}^{2}}\right)+\frac{1}{2}\left(\sigma_{\mathrm{v}}-\sigma_{\mathrm{h}}\right)\left(-\frac{4 \mathrm{R}^{2}}{\mathrm{r}^{2}}+\frac{3 \mathrm{R}^{4}}{\mathrm{r}^{4}}\right) \cos 2 \theta \\
& \sigma_{\theta}=\frac{1}{2}\left(\sigma_{\mathrm{v}}+\sigma_{\mathrm{h}}\right)\left(\frac{\mathrm{R}^{2}}{\mathrm{r}^{2}}\right)-\frac{1}{2}\left(\sigma_{\mathrm{v}}-\sigma_{\mathrm{h}}\right)\left(\frac{3 \mathrm{R}^{4}}{\mathrm{r}^{4}}\right) \cos 2 \theta \\
& \tau_{\mathrm{r} \theta}=\frac{1}{2}\left(\sigma_{\mathrm{v}}-\sigma_{\mathrm{h}}\right)\left(\frac{2 \mathrm{R}^{2}}{\mathrm{r}^{2}}-\frac{3 \mathrm{R}^{4}}{\mathrm{r}^{4}}\right) \sin 2 \theta .
\end{aligned}
$$

Equations (33-35) show that the induced changes in stress are confined to the rock adjacent to the hole, as would be expected from St. Venant's principle. At the boundary of the hole, $r=R$, these induced stresses have an important property: they are equal in magnitude and direction but opposite in sign to the virgin state of stress acting on that bouncary before the excavation of the hole. The sum of the induced state of stress and the virain state of stress yields that total state of stress everywhere including the stress-free boundary. This is the basis of integral methods for numerical solutions described later.

"In Figure 10 a solution to equations (30-32) is illustrated in the form of principal stress contours and trajectories, for $\sigma_{v}=1$ and $\sigma_{h}=$ $1 / 2$, in the upper right and left quadrants, respectively." 


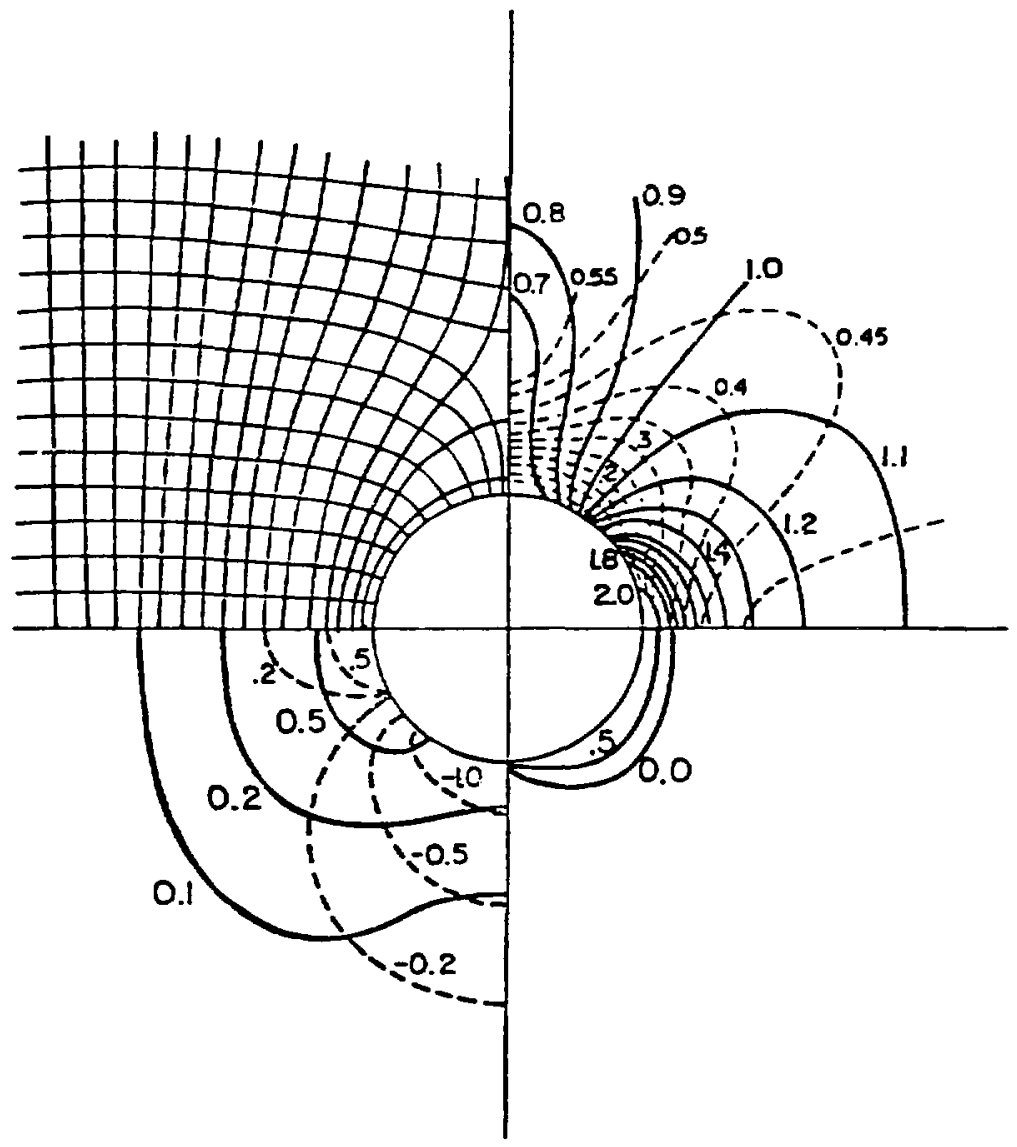

FIGURE 10. A sketch showing the results of Kirsch's solution for the stresses around a long circular hole subjected to a vertical principal stress with unit value and a horizontal principal stress of half this value. Contours of the values of the principal stresses are drawn in the left quadrant. In the lower right quadrant, are drawn contours of $\sigma_{1}-5 \sigma_{2}$, and in the left quadrant contours of the sums of, and differences between the principal stresses. 
These equations can be numerically inverted by generating a lookup table of their predicted results and then determining the initial stress/strain curve in the salt bed by interpolation.

This method has several advantages over the full mapping one:

1) Instead of experiencing the borehole distortion as a difficulty, it actually is used as the detection mechanism.

2) The measured regions are much closer to the coil with an inverse cube signal gain.

3) A considerable sample volume is integrated rather than only a small region as used in mapping.

4) The directionality requirements on the coil are released, owing to the high symmetricity of the borehole distortion pattern.

5) No need for computationally-complex, signal-to-noise reducing, zeugmatographic range resolution.

6) The volume of data is sharply reduced.

3) Operational Scenario

To pinpoint the wavelength to an accuracy of a fraction of a line, one needs at least two measured samples per bandwidth, high S/N, and a good interpolation routine. This reduces the necessity for scanning a great many points in the spectrum to determine the correct peak frequency.

A further cut in the number of separate measurements needed (each of which consumes about $1 / 2 \mathrm{~min}$ ) can be accomplished by using approximate estimates for the stress/strain and thus the frequency value. If one extrapolates from either the previous azimuth or depth, these estimates become fairly indicative unless the stress/strain has rapid spatial variations. In 
these cases, as little as 2-3 measurements are enough to accurately pinpoint the center frequency if the initial estimate came within +25 atmospheres of the correct value.

To resolv the azimuthal pattern may then require 4 to 8 measurements, but this again may be reduced by extrapolation from a different depth. In any case, 10-30 me ssurements, involving 10-15 minutes elapsed time, seem sufficient to haracterize a given depth element, and a complete stress/strain map of a boreh le would take under a day.

E. Description of an NQR Stress Sensor for Borehole Operation

The main subsy tems of an NQR borehole stress sensor are (Figure 11):

1) A manetic field generator, containing

a) A field solenoid

b) A power supply

c) A cooling system

2) An NIR-NQR double resonance transmitter-receiver, comprising

a) An NQR irradiator

b) A rotating transmit/receive coil

c) A matched, switched network and preamplifier

d) An NMR transmitter/receiver

e) A data system

1) Magn tic Field Generator

(a) Field soleriold. The purpose of this is to generate a - 1000 gauss field within the sodium chloride bed in the immediate vicinity of tive borehole wall. This coil must be moderately long, to acnieve reasonable axial 


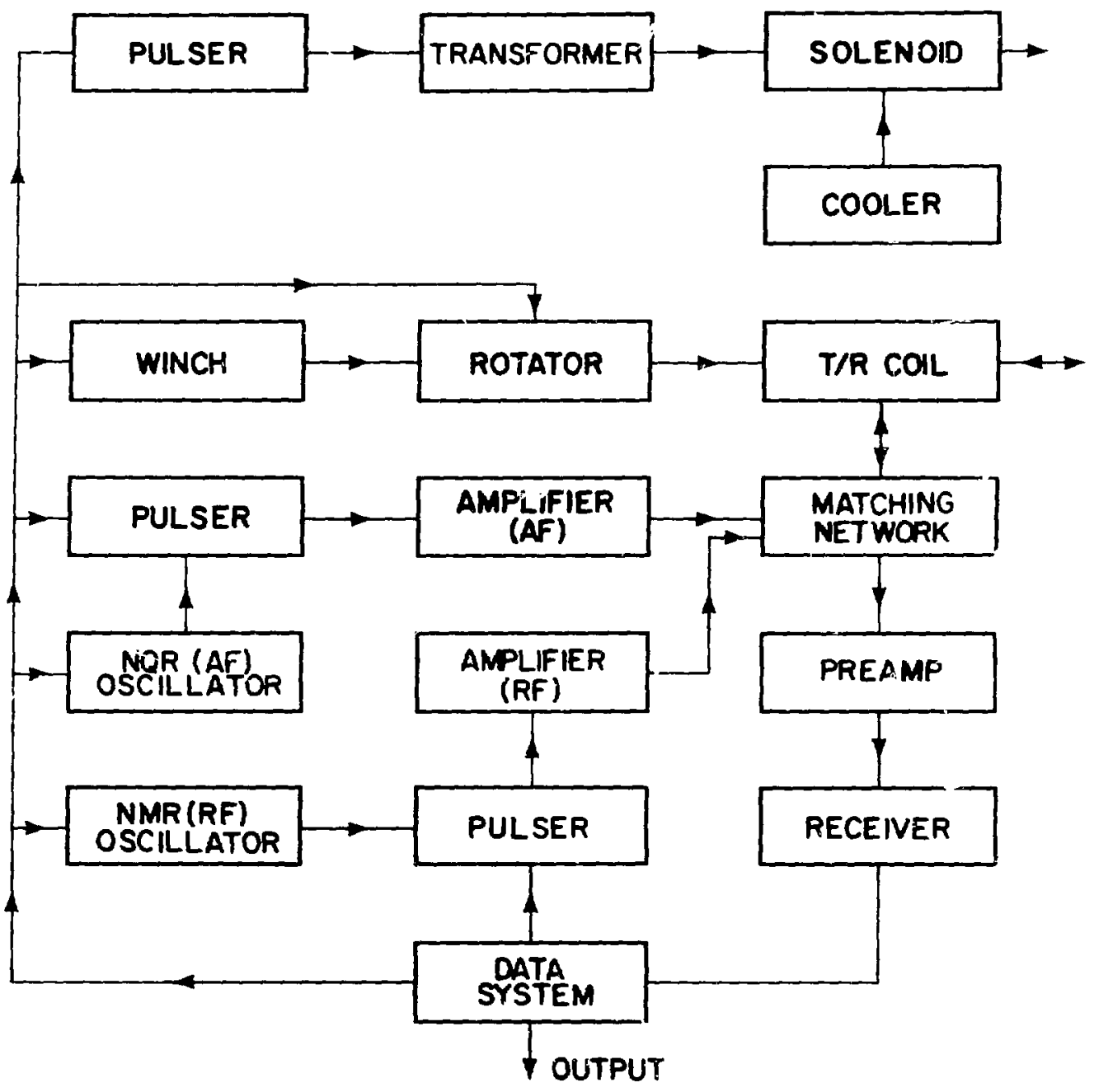

$\times B L 8012-2478$

FIGURE 11. BLOCK DIAGRAM OF NQR BOREHOLE STRESS SENSOR. 
honogenejty of the field around its center. In practice, further improvenent in this homogeneity may be possible by increasing the winding density or layering near the ends. A longer coil also increases the size of the instanteously observed sample volume and thus the signal-to-noise ratio (S/N). Eventually, the increased power requirement, which goes as the square root of the coil length, the physical dimensions of the system, the required depth resolution of the measurement, and the progressively closer approach of the coil to resonance with its on-off switching frequency, 1 imits the total length. A detailed calculatio of power requirements in the coil, assuming a $10 \mathrm{~cm}$ borehole diameter, indicates that for a coil length of $150 \mathrm{~cm}$ the peak power requirenent will be $100 \mathrm{~kW}$. Here the duty cycle including high, zero, and transfer field wait times $1 \cdot i 11$ be $25 \%$, indicating an overall power consumption of $25 \mathrm{~kW}$.

This power consumption is based on using standard, circular cross-section wire, with an optimum diameter of $5 \mathrm{~mm}$. A better solution uses spiral wound foil, giving not only reduced power consumption but a much higher resistance and thus a better match to a power source. Consider a coil wound of such strips with width $u$, thickness $v$, and length 1 . The number of turns, $n$, is given by

$$
\text { 1) } n=\frac{\ell}{v}
$$

and the resistance, $R$, is

$$
\mathrm{R}=\frac{2 \pi \mathrm{r}_{\mathrm{B}} \mathrm{n} \rho}{\mathrm{uv}}
$$


where $r_{B}$ is the radius of the strip and $\rho$ is the specific resistance. Substitution of $1 / v$ for $n$ gives

3)

$$
\mathrm{R}=\frac{2 \pi \mathrm{r}_{B} l \rho}{\mathrm{u} \mathrm{v}^{2}}
$$

Then from basic theory,

4)

$$
I=\sqrt{\mathrm{P} / \mathrm{R}}=v\left(\frac{\mathrm{Pu}_{\mathrm{u}}}{2 \pi r_{\mathrm{B}} \mathrm{Q \rho}}\right)^{1 / 2}
$$

where $I$ is the current and $P$ is power.

The central field of the solenoid, $H$, is

5)

$$
H=\frac{\pi u I}{5 r_{B} h}
$$

where $h$ is the height of the coil, while the field immediately exterior to its winding, $H_{0}$, is

6)

$$
H_{0}=\frac{\pi}{5} \frac{1}{v}
$$

which further gives

$$
\text { 7) } \mathrm{H}_{\mathrm{o}}=\frac{\sqrt{\pi}}{5 \sqrt{2}}\left(\frac{\mathrm{Pu}}{\mathrm{r}_{\mathrm{B}}{ }^{\ell}}\right)^{1 / 2}
$$

from which it can be shown that the input power required, $W$, is

8)

$$
W=\frac{50}{\pi} \quad\left(H_{0}^{2}\right) \quad\left(\frac{r_{B}^{l} \rho}{u}\right)
$$


If we use here the value

$$
\mathbf{u}=1.5 \mathrm{~cm}
$$

and the same values for the other variables as used for wire windings, then

$$
W=28 \mathrm{~kW} \text {. }
$$

This is a slightly more favorable value, which after application of the duty cycle, becomes

$$
W=7 \mathrm{~kW}
$$

Alternatively, higher values of $H_{0}$ for better $S / N$ may be considered by using a $25 \mathrm{~kW}$ input to the ribbon windings.

On the other hand, the effect of this change on $R, I$ and $V$ is large. The former values, for circular conductors, were

$$
\begin{aligned}
& R=0.165 \Omega \\
& I=776 \mathrm{~A} \\
& V=129 \mathrm{~V}
\end{aligned}
$$

For this improved conductor, and choosing

$$
v=0.20 \mathrm{~mm}
$$


we obtain

$$
\begin{aligned}
& R=27.2 \Omega \\
& I=32 \mathrm{~A} \text { (or } 1 / 4 \text { of this average) } \\
& V=865 \mathrm{~V},
\end{aligned}
$$

a much more favorable electrical condition, avoiding the need for a down-hole transformer and allowing simple power feeds via a reasonably low current cable.

Calculation of the inductance and self-resonance of such a coil shows it still to be compatible with rapid on-off switching.

Such coils do not, of course, give radially homogeneous fields, which limits the observation volume $V$ to the small values described above. These, however, are still large enough to give the desired $\mathrm{S} / \mathrm{N}$ with a substantial safety margin.

If even lower power consumption appears desirable (for which there is little demand) or higher $H_{0}$ 's are wanted (conservative design led to adoption of an easy to reach $H_{0}$ ), ferromagnetic inserts such as ferrites appear workable. An alternative, having no power consumption, but requiring a powerful winch for spatial rather than electrical switching, is a large permanent magnet. Here the required field is obtainable with greater simplicity but equal cost (large permanent magnets are expensive).

(b) Power supply. Transmitting power to the bottom of a borehole is much easier at the reasonable current and lower voltage of the strip wound coil. $1100 \mathrm{~V}$ power is readily available, being one of the standard power distribution voltages for which small, vehicle-mountable transformers are available if the 
motor generator operates at some other voltage. Complete with aii necessary insulation, an armored cable for this voltage and peak current, capabie of carrying the required current for 1-2 miles, is about 1/2" in diameter, and produces power losses not exceeding $20 \%$.

To generate the necessary power, a generator having about $50 \mathrm{~kW}$ power is necessary to cover the power losses, remaining electronic systems, etc. Mobile, vehicle-rated military power sources in this range typically operate at $440 \mathrm{~Hz}$ to achieve small size and low cost. Of course, the power supply to this coil must be rectified.

A much smaller power supply can be built using a resonant accuniulator topside. This would allow using a $15 \mathrm{~kW}$ power supply for the same job, but would require storage of about $100 \mathrm{~kJ}$ of electrical energy and be counterproductive in cost. A few $\mathrm{kW}$ of additional electrical power will be required at the sensor for operating all other systems and could best be provided by sending down electrical AC power over the same cable, and separating them with a small filtering transformer in the borehole. This filtering would be aided by the high impedance of the coil.

This power source must furnish power at two levels to the coil; one enough to produce $H_{0}=1000$ gauss, as discussed previously, as well as a "trickle" level of 5-25 gauss during which the NQR excitation transfers to the NMR domain. This trickle level is adjusted to scan from one stress/strain range to another. The switching between power levels must occur rapidly, roughiy as fast as the coil ringdown time, During the NQR excitation, the coil will be completely shut off. 
(c) Cooling system. The dissipation of an average of $-10 \mathrm{~kW}$ power at the bottom of the borehole would constitute an appreciable problem, as heating of the sample will shift its NQR frequencies as well as create new stresses. To avoid this, a coolant flow must extract this heat, mainly from the field solenoid.

To do this via water cooling, the coolant flow can be calculated ty

$$
G=0.004 \frac{\bar{p}}{\Delta t}
$$

where $\Delta t$ is the water temperature increase, $G$ its flow rate in liters/min, and $P$ the average power. If we now take

$$
\Delta t=10^{\circ} \mathrm{C}
$$

we arrive at

$$
G=4 \text { liters/min., }
$$

a quite comfortable flow rate.

2. NMR/NQR Double Resonance Transmitter -- Receiver

(a) NQR Irradiator. This essentially audiof requency oscillator-pulser amplifier chain serves to irradiate the NQR absorption frequencies in the sample. The absorbed energy then interacts with the NMR energy levels, during the transfer time, at which point the magnetic field is held at a level at which both transitions are at the same energy and resonate. 
The frequency coverage required here is 1 imited by the maximum pressure to be measured. If we assume this to be about $1000 \mathrm{~atm}$, some tens of $\mathrm{kHz}$ will be adequate. Since the bandwidth of the system is $\sim \mathbf{k H z}$, to measure the actual transition frequency a scan through about 40 resolution elements would be required for a stress/strain measurement.

In practice, each measurement will show some correlation with those taken at the previous azimuth and the same depth or the same azimuth and a previous depth, so that on the average a 3-5 point scan will be enough. The high pressure resolution of the measurement will actually come from high accuracy location of the NQR line center.

The power required here will be small, as saturation is easy to achieve at these low frequencies. Typically fields of a few gauss, requiring powers of a few hundred watts, will be enough.

(b) A rotating transmit/receive coil. This toroidal coil is wrapped around the main solenoid, and can rotate to produce a two-lobed cylindrical response pattern in the surrounding material. The orientation of this rotating coil at the maximum stress signal will fix the orientation of the borehole stress distortion pattern and thus of the original stress/strain. A typical configuration of such a coil is shown in Figure 12.

The toroidal coil must avoid self resonance at $1.1 \mathrm{MHz}$, the resonant frequency of sodium in $i k G$ (in order to have some slight tunability) and must achieve at the same time a $Q$ value higher than the $\sim 100$ limit imposed by the bandwidth and frequency of the measurement. The bandwidth, in turn, is imposed by the residual fluctuating magnetic field produced by the crystal lattice. The appendices describe this optimization in detail. For a radius of $10 \mathrm{~cm}$, a coil having resonance frequences $\gg 1 M H z$ and $Q>1000$ can be 


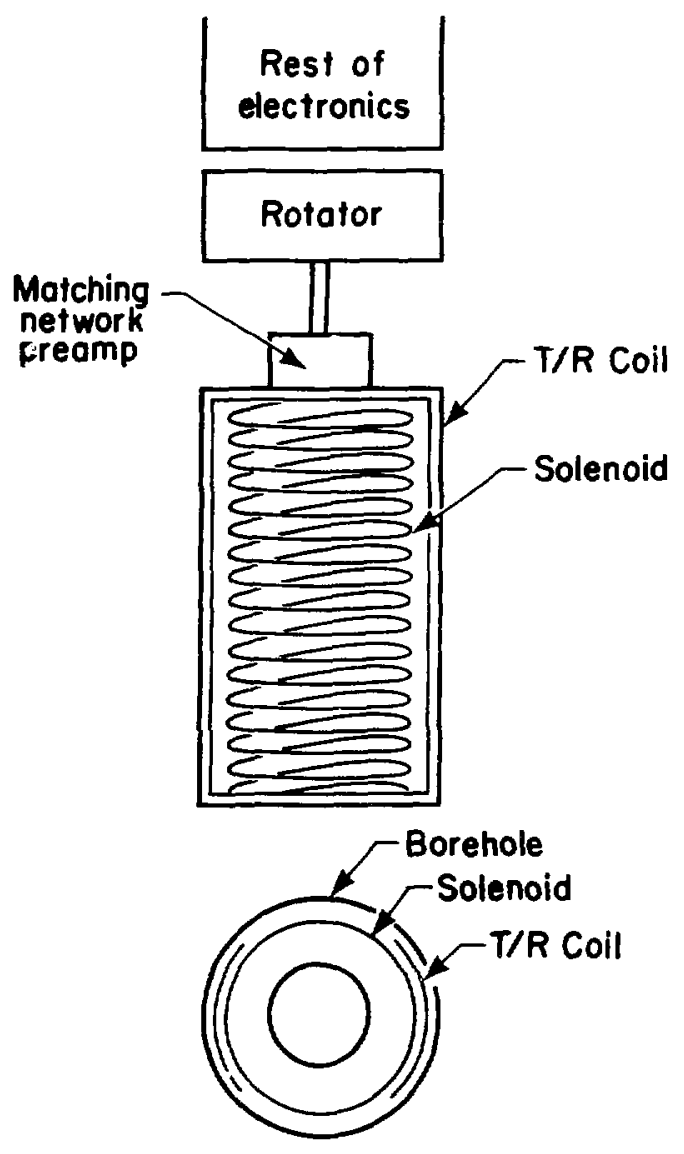

XBL $8012-2482$ 
built. Such Q's were obtained in previous work under contract with the U.S. Navy to develop an NQR landmine detector. (28)

There is no requirement for special guard cails to discriminate against background radiation. The coil will be well shielded from atmospheric noise deep underground. However, the need for rotating this coil in the gap between the so'enoid and the wall is a strong mechanical constraint. Since this coil must work both as a receiver and a transmitter it is coupled to an appropriate switching system.

(c) A matched switching network and preamplifier. Duplexing and recovery time of the transducer (switching) and circuit elenents cannot be readily separated since the circuit elements usually must be considered as part of the same network. The duplexing function cannot be considered complete until the effects of the transmit signal are reduced to a level below the received signal, or to a level where the two may be separated. The duplexing must limit the transmit signal to some maximum safe level specified at the receive terminals. The receiver isolation required by the duplexer is then directly proportional to transmitted power. In addition, the duplexer must provide a low loss path between the transmitter and the transducer during transmission and a low loss path to the receiver during reception.

As transmit power is increased, duplexing networks become more complex. Switching elements have some minimum realizable impedance and maximum power/current limitations. At the present no switching device is available suitable for series use in low level, low noise applications. Present devices are oniy low noise in the open state.

High power duplexing circuits are fairly complex band-pass-band-reject filters. Figure 13 illustrates some variations based on a simple filter 

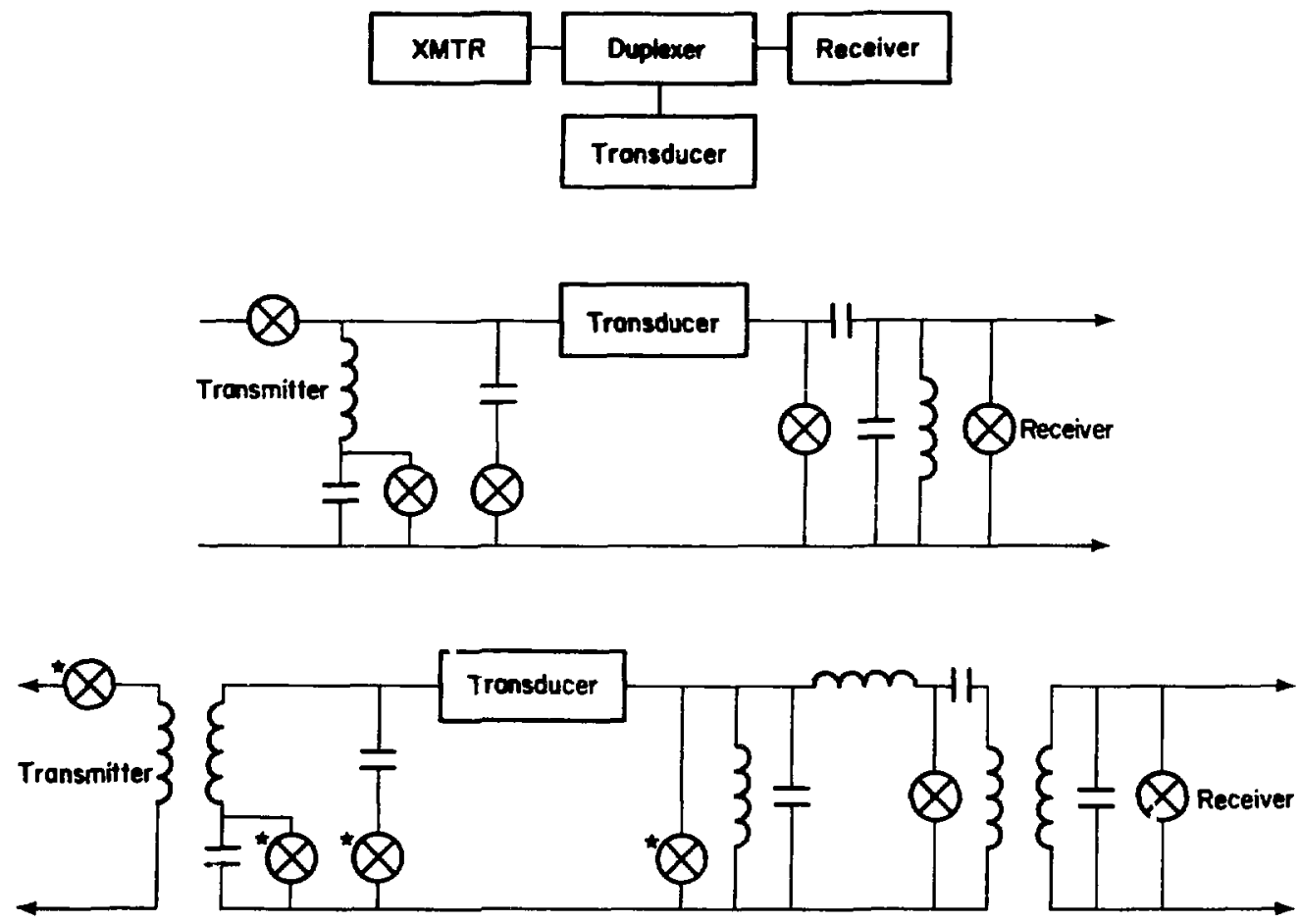

- High power switches

XBL $8012-2487$ 
design. Because of the increased complexity required for higher powers, losses are greater, affecting sensitivity and $Q$.

Gas discharge devices (TR's and ATR's) using hydrogen and water vapor are commonly employed in radar and in transmission line or wave guide analogues of the circuits illustrated. These are about the only devices presently available capable of handling the I swer levels probably required for the borehole instrumentation in the $10 \mathrm{MHz}$ region, and, particularly, in high $\mathrm{Q}$ coil configurations. The hydrogen-water-vapor jas discharge devices also have fast recovery time ( $\sim 5$ to 10 microseconds for multi-megawatt usage) but must be operated above the freezing point of water and certainly are not suitable for low temperature application. They are triggered when the voltage applied across the device exceeds the breakdown of the low pressure gas in them. In modern devices tritium is used to provide a low level ionization to reduce the breakdown voltage. When the applied voltage drops below the ionization potential, the discharge is extinguished. The addition of water vapor to the hydrogen decreases the deionization time.

The high power, high $Q$ circuits present a dequeing problem. A switching device is required with a hold-off voltage greater than the voltage present during transmission, but which can be rapidly turned on at the end of transmission and remain on while the circuits decay to a suitable level. Here either a modified hydrogen thyratron or a design using a longer recovery time gas discharge device (with a series resistor in parallel with it) can be used. Figure 14 is a simplified circuit illustrating this ar ach. Various circuit elements have shunting resistors switched ir $\because \quad$ critically damped conditions during the dequeing time.

Figure 15 is a simplified series resonant circuit with the curn-to-turn and other parazitic capacitances shown as one shunt capacitance across the 


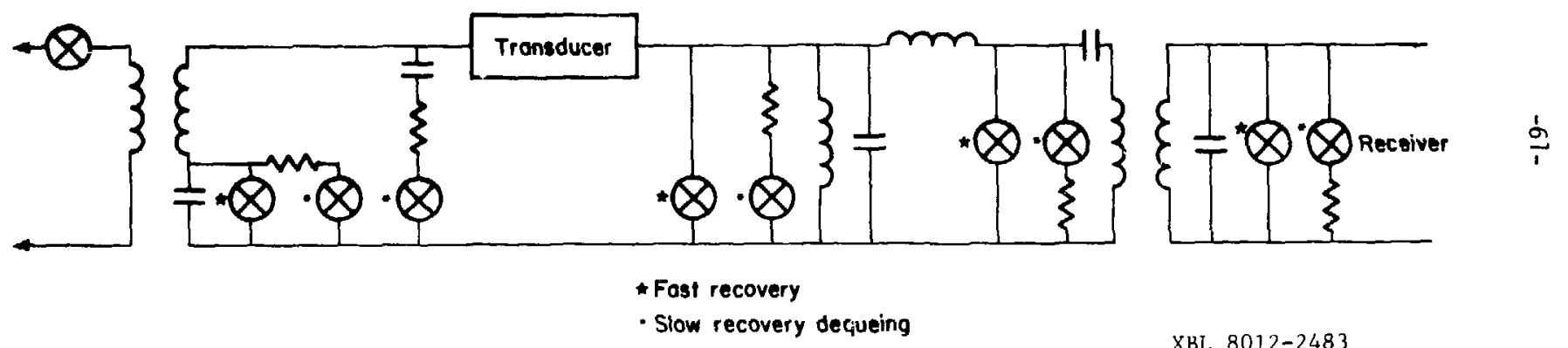

FIGURE 14. A HIGH POWER DEQUEING CIRCUIT 


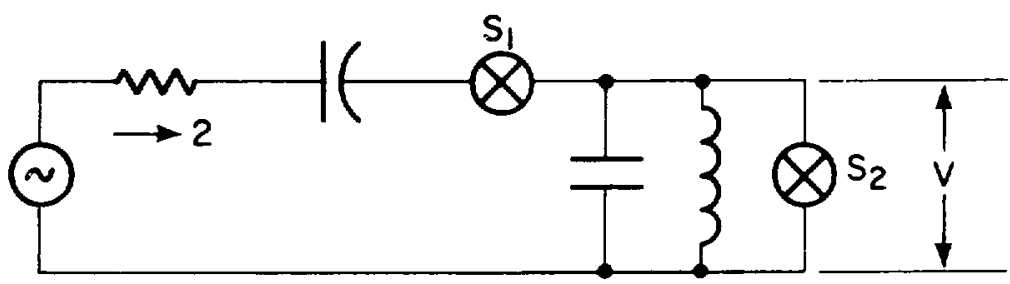

$\sim \sim$ Current if $S$, were olways ciosed

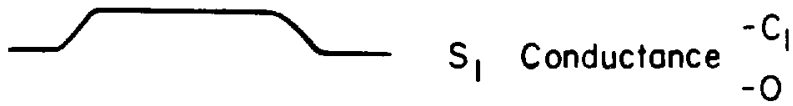

$\mathrm{S}_{2}$ Conductance ${ }_{-0}^{-\mathrm{C}_{2}}$

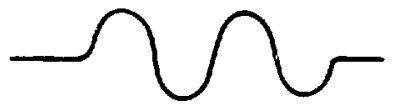

Current if $S_{1}-S_{2}$ conductances os show

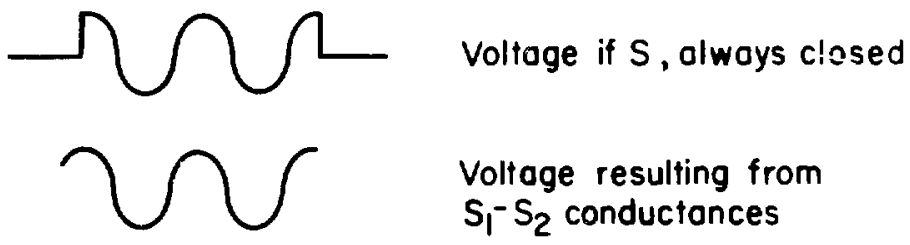

$X B L 8012-2477$ 
inductance. If the driving impedance is greater than the inductive reactance, the current through the circuit without a series switch would be as indicated. Some ringing is shown since this would be the case with any realizable source. With finite switching time and switch conductances controled so that conductances of the form indicated are realized, currents and voltages of the forms illustrated will be attained. Residual ringing and other non-ideal behavior is obviously going to bi: ient, but should be at greatly reduced levels.

Series switching could be employed with higher $Q$ circuits, but for the most part it is not necessary in laboratory instrumentation. For higher power applications it becomes very much a part of the transmitter design. Substantially better current waveforms shouid result from a series circuit that is at least critically damped without the switches.

The receiver canceling coils discussed previousiy accomplish essentially the same action as the series switch so that shunt dequeing is used to discharge the shunt circuit elements. Separate dequeing windings may be employed if good coupling can be obtained. In a particular jesign the benefits of DC isolation could be Targe.

Transmitter waveform through the switches must be such that no large low frequency componert in the nagnetic field is generated either by the series elements or by the dequeing action. The exciting magnetic field must be essentially an integral number of cycles or have leading and trailing matched positive and negative components so that there is not a low frequency or D.C. skew introduced. The Fourier transform of the exciting field should be symetric about the center frequency in the main lobe portion of the spectrum and th e should be no zero-frequency comporent. 
Wide bandwidth fast-recovery-time designed circuits should be employed in the receiver ahead of base-band conversion and band-limiting filters. Gating should be done as much as possible at wide bandwidths. Gating and limiting Defore filtering usually reduces the effects of large signals on the small signals imediately following and is a cormon techniquie used to reduce the effects of very large interfering signals.

Given these precautions in the switching systems, and the absence of background rf noise far urderground, a preamplifier whose noise is much lower than the coil's JohnsGil noise is practical, as can be seen in the Appendices.

(d) NMR transmitter/receiver system. This system embodies standard NMR technology with the following three exceptions:

1) It is designed for unusually low frequencies, which after the prearmifier imposes no significant system requirements.

2) It is designed to do time-gated MMR intensity measurements in a cyclical mode, instead of frequency scan or FFT operations in the pulsed mode. Actually, there is $\mathrm{n}$ NMR band to observe, since the inhomogeneity of the magnetic field spreads the NMR signal out over the entire spectrum. Specifically, the taking of one frequency sample by the filter bandpass in the system defines a volume in space which is the only one sampled since nowhere else dces $\mathrm{H}_{0}$ have the right value to put the $\mathrm{Na}^{23}$ resonance in the bandpass of the measurement. This is what makes the calculated volume so much smaller than the entire volume of sample near the coil, and is inciuded in our sensitivity calculations.

The time-gating in the MMR signal occurs at two levels. The first of these is that successive NMR measurements are taken at intervals, the cycle being exposure to high field, saturation of the MMR magnetization by an MMR 
pulse train, magnetic field shutoff, $H Q R$ irradiation, field increase to NMR-NQR resonance point, waiting for the exchange, and then going back to the full field, and completing the MMR measurement.

3) The NMR measurement is performed by a continuous train of very fast pulses following the SLSE sequence ${ }^{(29)}$, which sharply reduces the effect of $T_{1}$, and increases signal levels by allowing extensive signal averaging. This is the second level of timing control in the experiment.

(e) Data system. The data system must accomplish a number of tasks:

1) Clock, control, and monitor the pulsing sequence for the magnetic field solenoid, the NMR irradiation pulse sequence, and the NMR magnetization and readout pulse sequences.

2) Aid the operation of the switching transmit/receive network by real-time calibration and compensation of residuals.

3) Log depth, azimuth, temperature, and system field levels for function monitoring, data reduction, and calibration.

4) Accumulate repetitive signal for $S / N$ enhancement.

5) Sequence azimuth, rotate the coil, and initiate depth changes.

6) Control the "hi11-climbing" routine to zero in on an exact NQR frequency with a minimal number of tries.

7) Correlate the azimuthal reading pattern with value tables constructed from the borehole perturbation equations to reconstruct the original stress/strain values and directions.

8) Format all of the above for logging. 


\section{F. Predicted Sensitivities on Stress/Strain Determinations}

The calculations presented below refer specifically to sodium chloride and will have to be modified for other geologic specimens. The NMR signal from the three nuclei present in sodium chioride at a field of 1000 gauss occurs at frequencies of

$$
\begin{aligned}
& \mathrm{Na}^{23}-1126 \mathrm{kHz} \\
& \mathrm{CL}^{35}-417 \mathrm{kHz} \\
& \mathrm{CL}^{37}-347 \mathrm{kHz}
\end{aligned}
$$

Since signals in this frequency region tend to have an intensity that is linear in frequency, the $\mathrm{Na}^{23}$ NMR signal is the observable of choice for the double resonance measurements. The NMR sensitivity which is modified by the NQR transition can be calculated.

The signal induced in the sensing coil of an NMR spectrometer driven by an rf voltage $V_{0}$, and having a quality factor $Q$, to which the sample is coupled with an efficiency $\varepsilon$, is

1)

$$
\overline{\mathrm{V}}_{\mathrm{s}}^{2}=-4 \pi \epsilon \mathrm{Qx_{2 }} \overline{\mathrm{V}}_{0}^{2}
$$

where $x_{2}$ is the absorptive component of the nuclear magnetization.

Here, since the driving voltage is sinusoidal, we can substitute

2)

$$
\overline{\mathrm{V}}_{0}^{2}=\mathrm{V}_{0}^{2} / 2
$$


Furthemore, we can substitute

3)

$$
O=\omega L_{0} / R_{n}
$$

where $w$ is the frequency, $L_{0}$ the coil inductance, and $R_{0}$ its resistance, and we also have

4) $v_{0}=I_{B} R_{0}$

where $I_{0}$ is the coil current. Since the electric and magnetic fields in the coil store equal amounts of energy, we have

5) $\quad \mathrm{L}_{0} \mathrm{~J}_{0}^{2} / 2=\mathrm{VH}_{1}^{2} / 2 \pi$

where $V$ is the sensed volume and $H_{1}$ the $r f$ magnetic field intensity. We can now calculate

$$
\text { 6) } \quad V_{0}^{2}=\frac{V H_{1}^{2} R_{0}^{2}}{\pi L_{0}}
$$

and by substitution

7)

$$
\overline{\mathrm{V}}_{s}^{2}=2 \pi \mathrm{QVR} \mathrm{R}_{0} \omega\left(2 \epsilon \mathrm{H}_{1} \mathrm{X}_{2}\right)^{2}
$$

Here we have

8)

$$
2 \mathrm{H}_{1} \chi_{2}=\frac{\chi_{0} \omega_{0} \mathrm{H}_{1}\left(\omega_{0}-\omega\right) \mathrm{T}_{2}^{2}}{1+\left(\omega_{0}-\omega\right)^{2} \mathrm{~T}_{2}^{2}+\gamma^{2} \mathrm{H}_{1}^{2} \mathrm{~T}_{1} \mathrm{~T}_{2}}
$$


where $x_{0}$ is the static nuclear susceptibility, $\omega_{0}$ the resonant

frequency, $T_{1}$, the spin-lattice relaxation time, $T_{2}$ the spin-spin

relaxation time (equal approximately to the reciprocal of the intrinsic line width), and $y$ is the magnetogyric ratio of the nucleus used.

Equation 53 peaks at

9)

$$
\omega_{0}-\omega=\frac{\sqrt{1+\gamma^{2} \mathrm{H}_{1}^{2} \mathrm{~T}_{1} \mathrm{~T}_{2}}}{\mathrm{~T}_{2}}
$$

where

$$
2 H_{1} \chi_{2}=\frac{\chi_{0} \omega_{0} H_{1} T_{1}}{2 \sqrt{1+\gamma^{2} H_{1}^{2} T_{1} T_{2}}}
$$

In practice, to optimize the signal we saturate its intensity by letting $\mathrm{H}_{1}$ grow unt j1

11)

$$
H_{1}^{2}=\frac{1}{\gamma^{2} T_{1} T_{2}}
$$

at which point

12)

$$
\omega_{0 \mathrm{PT}}=\omega_{0}
$$

13)

$$
2 H_{1} x_{2}=\frac{x_{0} \omega_{0} T_{2}}{2 \gamma \sqrt{T_{1} T_{2}}}
$$

giving by substitution 
14)

$$
v^{2}=\frac{\pi U V R_{0} \omega_{11} r^{2} n_{11}^{2} I_{11}^{2} T_{2}}{2 T_{1}}
$$

This noise must be detected against the receiver noise, which if well designed, is Johnson-noise limited by the receiver coil. This is given by

$$
\bar{v}_{n}^{2}=2 R_{10} k I_{1} I_{i}
$$

where $V_{n}$ is the noise voltage, $k$ is Boltzman's constant, $T_{c}$ the coil temperature, $t$ the measurement time, and $F$ is the factor by which the receiver noise exceeds the sensing coil's Johnson noise. This gives

$$
V_{s}^{-2} / V_{n}^{2}=\frac{\pi Q V \omega_{0} \epsilon^{2} \chi_{0}^{2} H_{0}^{2} T_{2} t}{4 k T_{i} F T_{1}}
$$

Here we can calculate the $D C$ nuclear paramagnetic susceptibility

$$
x_{0)}=\frac{N 4 \pi^{2} \gamma^{2} I(1+1) h^{2}}{3 k T_{s}}
$$

where $I$ is the nuclear spin of the target nucleus, $T_{S}$ the sample temperature, $N$ the number density of the target nucleus, and $h$ is $P l a n c k$ 's constant.

From the above we can calculate the $\mathrm{rms} S / N$ ratio as

$$
\bar{V}_{\mathrm{c}} / \mathrm{V}_{\mathrm{n}}=\frac{\mathrm{Q}^{1 / 2} \mathrm{~V}^{1 / 2} \epsilon \mathrm{h}^{2} \mathrm{~N} \gamma \mathrm{I}(\mathrm{I}+\mathrm{I}) \mathrm{t}^{1 / 2}}{6 \sqrt{2} k^{3 / 2} \mathrm{~T}_{\mathrm{s}} \mathrm{T}_{\mathrm{c}}^{1 / 2} \mathrm{~F}^{1 / 2}}\left(\mathrm{~T}_{2} / \mathrm{T}_{1}\right)^{1 / 2} f_{0}^{3 / 2}
$$

where $f_{0}$ is the resonant frequency. We can also express this as 
19)

$$
\vec{V}_{s} / V_{n}=\frac{Q^{1 / 2} V^{1 / 2} \epsilon h^{2} \mathrm{~N} \gamma^{5 / 2} I^{1 / 2} I(1+1)}{24(\pi \mathrm{k})^{3 / 2} \mathrm{~T}_{\mathrm{s}} T_{c}^{1 / 2} \mathrm{~F}^{1 / 2}}\left(\mathrm{~T}_{2} / \mathrm{T}_{1}\right)^{1 / 2} \mathrm{H}_{0}^{3 / 2}
$$

In order to calculate $Q$, we must refer to the coil design description in the appendices, where it is shown as a function of coil diameter. In practice, of course, loading of the coil by the receiver and the cross coupling filters required limits $Q$, but values $>1000$ should be attainable at these low frequencies. (28)

The useable volume, on the other hand, is determined not by the effectively infinite sample volume, but by the magnetic field gradient around the exciting solenoid. In the immediate vicinity of the solenoidal field coil's periphery, the field falls off with the inverse square of the radius, making the radial depth element sampled equal to

$$
\text { 20) } \Delta_{r}=\frac{{ }_{B} B_{W}}{2 \gamma H_{0}}
$$

where $r_{B}$ is the borehole radius and $B_{W}$ is the bandwidth.

From here we get the total sampled volume as

$$
V=\frac{r_{B}^{2} B_{w} \alpha l}{4 \pi \gamma H_{0}}
$$

where $\alpha$ is the utilized fraction of the circumference, and $\ell$ is the length of the sampled region.

We can now tabulate a few of the relevant properties of $\mathrm{Na}^{23}$ : 


$$
\begin{aligned}
& I=3 / 2 \\
& Y=1126 \mathrm{~Hz}_{\text {gauss }}^{-1} \\
& \mathrm{~N}=2.23 \cdot 10^{22} \mathrm{~cm}^{-3}
\end{aligned}
$$

A further substitution possible is

$$
T_{2}=1 / B_{w}
$$

In order to calculate the value of $E$, the geometry of the sensing coit must be taken into account. This must lie outside the solenoid which generates $H_{0}$, which, in order to generate the largest possible $H_{0}$ with a reasonable current, must fill the borehole. This restricts the coil to a flat loop surrounding the solenoid. To maximize the efficiency with which we generate $H_{0}$, and to maximize the sample volume $V$, we elongate the solenoid and give the receiver coil a rectangular cross section. In order to increase the coupling of the coil to the sample, its solid angle as perceived from the sample must be maximized. This can be done by giving the coil a toroidal cross section utilizing more of the borehole's circumference. The limit to this improvement is reached when the restriction on $\alpha$ by this procedure begins to dominate the total signa1. Actual integration of the resulting function shows an optimum toroid width equal to the borehole radius. As perceived by the sample, the transmit/receive coil is then a short on-axis rectangular solenoid.

The extent of the coupling of the sample to such a coil is the exact inverse problem of calculating the field induced by the coil at the sample's location. The ratio of the field produced by the coil at its center (at which 
location $\epsilon \rightarrow 1)$ to that produced at the actual sample location is thus the inverse of $\epsilon$.

We can now write for the axial field of a circular solenoid (an approximation adequate for these purely relative calculations) of radius a, length $b$, at a distance $c$ from its center along the axis

$$
\mathrm{H}_{\mathrm{s}}(\mathrm{c})=\pi / 5 \mathrm{nIh}\left[\frac{0.56+c}{\sqrt{\mathrm{a}^{2}+(0.56+c)^{2}}}+\frac{0.5 t}{\sqrt{\mathrm{a}^{2}+(0.56-c)^{2}}}\right] \text {. }
$$

In the above case, we can write at the sample

$$
a=\sqrt{3} / 2 r_{B}
$$

giving

$$
H_{i}(c)=\pi n l / r_{B}(\sqrt{3}-1) / 10
$$

while for $c \equiv 0$ we have

27)

$$
H_{s}(0)=\pi n l / 5 r_{B}
$$

giving for the efficienry

$$
E=(\sqrt{3}-1) / 2=0.366
$$


In order to calculate $\mathrm{H}_{0}$, we calculate the field of the main $\mathrm{DC}$ solenoid along the outer edge of its winding by

29) $\quad \mathrm{H}=\frac{\pi n \mathrm{I}}{10 \mathrm{r}_{\mathrm{B}} \mathrm{h}}$

where $h$ is the solenoid's length/diameter ratio and $h \gg>1$. Then

30)

$$
h=V / 2 I_{B}
$$

If we now assume this solenoid contains closely-wound layers of wire having a diameter $d$ and a resistivity $\rho$, we have

31)

$$
R=\frac{x \rho r_{B} n}{d^{2}}
$$

and

$$
n=\frac{\ln }{d}
$$

where $m$ is the number of coil layers, giving

33)

$$
R=\frac{x \rho r_{B} l m}{d^{3}}
$$

This number will usually be low enough to require a downhole transformer rectifier. If we now substitute the above and

$$
l=\sqrt{\mathrm{P} / \mathrm{R}}
$$


where $P$ is the peak power during the high field portion of the double resonance cycle, we have

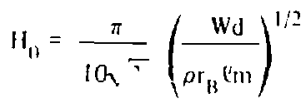

To calculate the proper value of $T_{1}$ to use in the calculations, the precise pulse sequence used in the NMR measurement is of importance. An optimal sequence for use here is the SLSE multiple spin-locked spin-echo method described in the appendices, which does better than the pure $T_{1}$ 1 imited system by the factor.

36)

$$
1=\eta^{1 / 2}
$$

where $n$, the numbsr of pulses in the train, is of the order of $10^{2} \mathrm{~T}_{2}$. Finally, Q will generally be limited by the signal bandwidth at

$$
0=\frac{\gamma H_{0}}{B_{u}}
$$

Combining all of the above, we can write for equation (64)

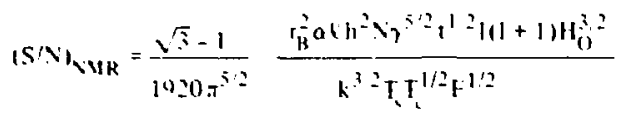

Here it is reasonable to assume $T_{s}=T_{c}=300^{\circ} \mathrm{K}$. F can be assumed to be of order unity, although slight adjustments for finite measuring times, as described in the appendices, would be required.

The actual NQR signal, detectable as a modification of the NMR one, has a $S / N$ prodortional to the latter. Here the extent of the perturbation if the 
measured quantity, and it is desirable to bias this into the range where convenient measurements can be done.

For the present application the energy transfer between NQR and NMR must be optimized, by providing enough time for this transfer to occur within the overall time constraint imposed by the $T_{1}$ relaxation. Here we take advantage of the resonant enhancement of this transfer process at the intermediate magneti: field where the NQR and NMR energies are identical. For this purpose the "OC" magnetic field used is switched in a 3-level zycle containing this interinediate value as well as 0 and the high one 1,000 gauss), with fast transition in between. At this optimum coupli is the $S / \mathbb{N}$ in NQR would still te l/e that of its NMR carrier. The optimum va'ue of $t$ is limited to somewhat less than $T_{1}$, and for the $T_{1}$ values expect. $d$ would be of the order of $10 \mathrm{sec}$.

The meaning of the abovi equations can now be clarifier tnrough an example. Consider a situation where

$$
\begin{aligned}
H_{0} & =1000 \text { gauss } \\
r_{B} & =10 \mathrm{~cm} \\
G_{W} & =10^{3} \mathrm{~Hz} \\
2 & =\pi \text { (for a bilaterall r-symmetric measurement) } \\
= & 150 \mathrm{~cm} \\
v & =1126 \mathrm{~Hz} \mathrm{gauss}^{-1} \\
1 & =1.73^{\prime \prime} 10^{-6} \therefore \mathrm{cm}^{-1} \text { (copper) } \\
T_{1} & =30 \mathrm{sec}^{-3} \mathrm{sec} \\
T_{2} & =10^{-3} \mathrm{sec}
\end{aligned}
$$




$$
\begin{aligned}
& m=1 \\
& d=0.5 \mathrm{~cm}
\end{aligned}
$$

Then

$$
\begin{aligned}
\omega_{11} & =1.126 \mathrm{MHz} \\
H_{1} & =0.005 \text { gauss } \\
Q & \geq 1126 \\
V & =3.33 \mathrm{~cm}^{3} \\
S / N_{N M R} & =880 \\
S / N_{N Q R} & =320
\end{aligned}
$$

Our ability to locate precisely the center of a peak, $E_{f}$, depends on the $S / N$, and is approximately

$$
\text { 39) } \quad F_{f}=\frac{B_{w}}{S i N}
$$

giving in our example

$$
\text { 40) } \quad F_{f}=3.0 \mathrm{~Hz}
$$

which even for the lowest stress coefficients represents a pressure noise of 0.16 atmospheres.

This is, of course, a mere calculation, and conservative engineering practice requires a margin of safety of 10 above this, leading to a pressure noise level of $1.6 \mathrm{~atm}$. 


\section{CONCLUSIONS AND RECOMMENDATIONS}

\section{A. Conclusions}

The research and development performed to date yielded some very bar.information:

(1) The NQR technique should be able to resolve changes in triaxial pressures in salt of 25 psi or smalier and determine their principal direct"ins.

(2) There are no obvious thcoretical problems at this time.

(3) Downhole instrumentation, suitabie for in-situ determination of pressure appears to be within the state-of-the-art.

(4) Detailed laboratory measurements on salt, under known pressures (magnitude and direction), are needed to refine the theoretical calculations and to permit the design of fieldable hardware.

\section{B. Recommendations}

Based on work done to date, the recormendations can be divided into two catagories -- additional work with salt and initiation of research on aluminum-27 containing rocks. For salt the suggestions are:

(1) Sufficient laboratory measurements on salt to permit data to be statistically evaluated on the effect of pressure and its direction on the NQR spectrum.

(2) For measurements in the borehole, existing calculations will have to be transformed from Cartesion to polar coordinates. 
(3) Calculation of line shapes associated with salt under various applied pressures and comparison of these with data com controlled laboratory experiments.

(4) Evaluate the effect of point defects and dislocations on the electic field gradient and the line shape.

(5) Finalize the design of a laboratory NQR system which will permit optimi. measurements of salt under applied directional pressure.

.u) Construct the instrument defined by (III.B.5)

(7) Determine the effects of such variables as temperature, chemical, and crystallographic inhomogeneities and plastic flow on the accuracy of the stress/strain NQR measurements.

For aluminum-27 containing geologic samples the following must be done:

(8) Locate the aluminum-27 NQR resonances in Al-0 and Al-0-Si type compounds.

(9) Measure the NQR spectra of select AT-O-Si compounds as a function of direction and pressure applied to the sample.

(10) Make a preliminary calculation as to the feasibility of measuring stress/strain in aluminum-27 compounds using data from (III.B.9).

(11) Evaluate contributions of such factors as multiple aluminum-27 compounds, temperature, crystallographic inhomogeneities, and defects and dislocations on stress/strain measurements.

(12) Repeat (III.B.5 and 6) for the aluminum-27 system.

It should be noted that the most beneficial and cost effec:ive program is one where the $\mathrm{NaCl}$ and aluminum-27 investigations are done in parallel. Inasmuch as the salt studies are far ahead of the aluminum ones, and will, in all probability stay that way, the results for the less complicated salt system can be used as a guide for the more complex aluminum-27 research and development. 


\section{REFERENCES}

1. Love, A.E.H. A Treatise on the Mathematical Theory of Elasticity, Dover, New York (1944).

2. Jaeger, J.C. and Cook, N.G.W. Fundamentals of Rock Mechanics, Chapman and Hall, London (1976).

3. Leeman, E.R. Rock Mechanics $\underline{3}, 25-60$ (1971).

4. Rocha, M. and Silverio, A. Geotechnique 19, 116-132 (1969).

5. Blackwood, R.L. Proc. Serni. Symp. of Field Measurements in Rock Mechanics, Zurich, Vol. I, pp. 137-150 (197\%).

6. Duncan-Fama, M.E. and Pender, M.J. Internl. J. Rock Mech. Min. Sci. and Geomech. Abstr. 17, 137-146 (1980).

7. Baar, C.A. Applied Salt - Rock Mechanics, I. The In-Situ Behavior of Salt - Rocks, Elsevier, New York (1977).

8. Marsh, J.L., Jr. and Casabel1a, P.A. Phys, Rev. 150, 546-551 (1966).

9. Schempp, E. and Bray, P.J. "Nuclear Quadrupole Reconance Spectroscopy" in Physical Chemistry, and Advanced Treatise, Vol. 4, Eyring, Jost and Henderson, eds., Academic Press, New York (1970).

10. Nye, J.F. Physical Properties of Crystals, Clarendon Press, Oxford (1972).

11. Shulman, R.G., Wyluda, B.J. and Anderson, P.W. Phys. Rev. 107, 953-958 (1957).

12. Kanert, 0. and Mehring, M. "Stat ic Quadrupole Effects in Disordered Cubic Solids" in NMR Basic Principle and Progress, Vol. 3, Diehl, Fluck, and Kosfield, eds., Springer-VerTag, New York, (1971).

13. Lucken, E.A.C. Nuclear Quadrupole Coupling Constants, Academic Press, New York (1969).

14. Muehliserger, W.R. "Internal Structure of the Gra I Saline Salt Dome, Van Zandt County, Texas." Univ. Texas Bus. Econ. Ge siogy Dept. Inv. 38, 18p (1959).

15. Kern, H. Tecionophysics $\underline{39}, 103-120$ (1977).

16. Carter, N.L. and Heard, H.C. Am. J. Sci. 269, 193-249 (1970).

17. Kamb, W.B. J. Geology 67, 153-170 (1959).

18. Nichols, E.A. Am. Inst. Min. Meta17. Petroleum Engineers, Petroleum Devel. and Tech. Trans. 170, 44-50 (1947). 
19. Moses, P.L. Worid 0i1 152, 79-82 (1961).

20. Anderson, L.0. and Fors 1 ind, E. Arkiv Fysik 28, 49-64 (1964).

21. Cohen, N.H. and Reif, F. "Quadrupole Effects in Nuclear Magnetic Resonance of Solids," Solid State Physics, Vol. 5, Seitz and Turnbu11, eds. Academic Press, New York (1957).

22. Lowe, I.J. and Norberg, R.E. Phys. Rev. 107, 46-52 (1957).

23. Koo, J.C. Ph.D. Thesis, Univ. of California, Berkeley (unpublished), (1970).

24. Hahn, E.L., Koo, J.C., and Hsieh, Y. Chem. Phys. Letters 13, 563-566 (1972).

25. Edmonds, D.T. Phys. Reports $\underline{\text { C29 }}$, 233-290 (1977).

26. Edmonds, D.T. and Mailer, J.P.G. J. Mag. Resonance 26, 93-99 (1977).

27. Klainer, S.M., Hirschfeld, T.B., and Marino, R.A. Fourier, Hadamard, and Hitbert Transforms in Chemistry, A. Marshal1, ed., PTenum, New York (in press).

28. Harding, J., Hirschfeld, T. B., KTainer, S. M., Wade, D., and Marino, R. A. "NOR Detection Studies", Final Report to Naval Electronics Laboratory Center, San Diego, March, 1976.

29. Marino, R. A. and Klainer, S. M., J. Chem. Phys. 67, 3388-89 (1977). 


\section{A-1}

\section{APPENOICES}

\section{A. Practical Applications of FT-NQR Spectroscopy}

(to be published as Ref. 27)

(The Appendices which follow give some very specialized and technical discussions. Appendix A provides a general overview of recent developments in NQR FT spectroscopy. Appendices $B-H$ are the result of highly complex calculations on coil and antenna designs and may be of interest only to the specialist. Whether all these results will be immediately applicable to the borehole-stress problem has not been determined because the final system has not been completed.) 


\title{
PRACIICAL APPLICATIONS OF FT-YQR *
}

by

\author{
Stanley H. Klalner, *t Tonas B. Hi rschfeldt \\ and Robert A. Marinot+
}

\section{INTROUUCTION}

FT-NQH (Fourier Trantorm Nuclear guadrupole Resonance) spectroscopy is one of the newer analytical tools. Although nuclear quadrupole coupling constants were first observed in acoms by Schmidr and Schuler(1) in 1935 and in molecules by kellog(2) and coworkers 1n 1936, it was the discovery of pure quadrupole coupling transitions in solids by Dehmelt and Kruger(3)(4) in 1950 and 1951 that initiated the inderstanding of ine NQR phenomenon as presently ut1lized.

Duriag the past $3 !$ YHers the growth of NQR has been anything but phenomenal. A great quantity of theoretical work has been reported but the experiwental investigations have been restricted prifarlly to nitrogen-containing and haloyenated compounds. How, then, can an application paper, such as this De justifled? The answer lies in responding to two specific questions:

(a) What has hindered the growth of NQR to date and can this be overcome with present day technology?

* In1s work was supported in part by the U. S. Department of Energy under Contract No. W-7405-ENG-48 and U. S. Army Reseaxch office under Contrac'́ No. DAAG-29-7y-0025.

**Lawrence Berkeley Laboratory, 90/1140, Berkeley, CA 94720.

+Lawrence Livermore National Laboratory, L325, LIvermore, CA 94550.

Hunter College of CUNY, 695 Park Avenue, New York, N.Y. H0021.

(1) H. Schuler and T. Schmidt, Z. Phys. 94,457 (1935).

(2) J. 11. H. Kellog, I. I. Rab1, N. F. Ramsey and J. R. Zacharias, Phys. Rev. 느, $728(1936)$.

(3) H. G. Dehmelt and H. Kruger, Naturwss. 37, 111 (1950).

(4) H. G. vehnelt and H. Kruger, Naturwiss. 38, 921 (1951). 
(b) Why is it inportant that Nuk be developed as a practical amalytical tecnnique ac this the?

In responding to these queries, it is possible to show that FI-NQR is ootn needed and inplewentable.

This paper $1 \mathrm{~s}$ directed at the applied scientist, and, therefore, it aves not contaln the in-depth theoretical and tathematical information of fnterest to the research scientist. Texts by Schempp and Bray(5), Lucken(6), slichter (7) and B1ryukov, Voronkor and Saf1n(8) can supplenent the 1nformation on NUR presented in this paper. There is, however, no primer on FI-NQR and the Dasic concepts of this specifi. "Tea will be addressed in this discussion. i. LVUK SPECTROSCOPY

Nuclear (uadrupole Resonance (NQR) is a branch of radio-frequency spectroscopy. The NuR spectroneter detects the interaction of a nuclear quadrupole woment with the electric fleld gradient (EFG) produced by the charge distriDution in a solid state compound. The quadrupole monent artses because the nuclear charge distribution is nonspherical. Resonance occurs when transitfons froun one spin state to another are exclted by radio-frequency electro-magnetic oscillacions. In this way, NQR is quite simlizr fir princlple to nuclear

(5) E. Scheppp and P. J. Bray, "Nuclear Quadrupole Resonance Spectroscopy", Pnysical Chemistry, an Advanced Treatise, Volume 4, Academic Press, New York (1970).

(6) E. A. C. Lucken, "Nuclear Quadrupole Coupling Constants", Academic Press, New York (1909).

(7) C. P. Slicnter, "Principles of Magnetic Resonance", Second Edition, Springer Series in Solid-State Sciences 1, Springer-Verlag, Berlin, deidelberg, New York (1979).

( $($ ) I. P. Biryukov, M. G. Voronkov and I. A. Safin, "Tables of Nuclear Yuadrupole Resonance Frequencies", Israel Program for Scientific Translations, Jerusalem (196'y). 
angetic resonance (NRR). In WQR, however, the energy levels depend on the coupling of the nuclear monent to the interagl electric field gradient, whereas in WMR they are prinarily dependent on the coupling of the nuclear magnetic moment with an external magnetic field. Since the EFG is a very sensitive function of the molecular and crystal structure, the resonance frequencles and band shapes are specific to each compound, and NQR data can be used for determining unambiguous sample identification, local electronic structure, atoml: arrangement, order/d1sorder phenomena, and crystal phase transformations. In addition, molecular dynamics in the solid state can be studied. Furthermore, since NQR data are gensftive to changes in temperature and pressure, there 13 the possibility of obtaining strain information.

\section{A. Basic Concept of NQR}

In order to understand the orfgin of nuclear quadrupole resonance, it is usual to visualize the nucleus as a classical distribution of positive charges, $\rho_{N}(\vec{r})$, over a volume of characteristic dimensions on the order of nuclear radf1, 1.e. $10^{-13} \mathrm{~cm}$ (F1gure 1 ). On the other hand, a charge cloud extending over a volume on the order of several angstroms $\left(10^{-8} \mathrm{~cm}\right)$ generates an electrostatic potential $\phi(\vec{r})$ which can be considered as varying slowly over the region occupled $y$ the nucleus. The electrostatic energy $w$ of the system can be expressed ( 7 ) as:

$$
=\quad \int \rho_{N}(\vec{r}) \phi(\vec{r}) d \vec{r}
$$

which can be expanded in terms of the moments of the nuclear charge distribution:

$$
w \quad=-\phi(0) q_{N}+\vec{p} \cdot \frac{E}{E}(0)-\frac{1}{6} \sum q_{1 J} q_{1 J}+\ldots
$$


where ( 0 ) aeans evaluated at the origin, the center of anss of the nucleus, and

$$
\begin{array}{ll}
q_{N} & -\int \rho_{N}(\vec{r}) d r \\
\vec{p} & -\int \rho_{N}(\vec{r}) \vec{r} \overrightarrow{d r} \\
Q_{1 j} & =\int \rho_{N}(\vec{r}) x_{1} x_{j} d \vec{r} \\
\vec{E} & =-\vec{\nabla} \phi \\
q_{1 j} & =\left(\partial E_{1} / \partial x_{j}\right)_{0}
\end{array}
$$

muclear charge

nuclear dipole monent vector

nuclear quadrupole nonent tensor

external electric field vector

external electric field gradient tensor

From quantum mechanical considerations it can be proven that the only non-vanishing terms in Eqn. 2 are the first, thitd, and other odd-ordered ones. The first term is simply a constant, and, therefore, the orlentationalydependent term in the energy is the quadrupolar energy (hexadecapole interactions are extremely small if they exist at all):

$$
W_{Q}=\frac{1}{6} \sum_{1 j} q_{1 j} Q_{1 j}
$$

$Q_{f j} 1 s$ diagonal and 1 s related to the componerits of the nuclear spin $I_{1}$ according to:

$$
Q_{11}=\frac{e Q}{I(2 I-1)}\left[3 I_{1}{ }^{2}-I(I+1)\right]
$$

where $I=\left(\sum I_{1}^{2}\right)^{1 / 2}$ is the nuclear spin and eq 18 defined as the nuclear quadrupole woment. Since $\nabla<\phi=0$ by the Laplace equation, we have

$$
\sum_{1} q_{11}=0 \text {. }
$$

The quantum mechanical expression for the quadrupols energy (the Hamiltonian) takes the particularly simple form:

$$
E_{Q}=\frac{Q Q}{2 I(2 I-1)} \sum_{1} q_{11} I_{1}^{2},
$$

which can also be expressed in the form: 


$$
B_{Q}=\frac{e^{2} g Q}{4 I(2 I-I)}\left[\left(3 I_{z}{ }^{2}-I^{2}\right)+n\left(I_{x}{ }^{2}-I_{y}{ }^{2}\right)\right]
$$

where $I$ and $I_{j}$ are spin operators.

The quantity $e^{2} q Q$ is called the quadrupole coupling constant of the system and $\eta$ is defined as the asymetry parameter of the electric fleld gradient (EFG):

$$
\begin{aligned}
& n \equiv \frac{q_{x x}-q_{y z}}{q_{z z}} \text {, and } \\
& e q \equiv e_{z z} \text { so } e^{2} q Q=(e Q)\left(e q_{z z}\right)
\end{aligned}
$$

when the axes are labelled

$$
\left|q_{z z}\right|>\left|q_{y y}\right|>\left|q_{x x}\right| \text { by convention. }
$$

For nitrogen-14, wth nuclear spin $I=1$, the solution of the Schroedinger equation $H_{Q} \Psi_{N}=E_{N} \Psi_{N}$, where $\Psi_{N}$ is the nuclear wave function, can be shown to lead to a three level system of energies given by

$$
\begin{aligned}
& E_{z}=e^{2} g_{Q / 2} \\
& E_{x}=-E_{z}(1-n) / 2 \\
& E_{y}=-E_{z}(I+n) / 2
\end{aligned}
$$

Transitions between these levels can be induced with oscillating magnetic flelds of the proper (resonant) frequencles (Figure 2). The frequencies of thege transitions are

$$
\begin{aligned}
& v_{+}=\frac{e^{2} g Q}{4 h}(3+n) \\
& v_{-}=\frac{e^{2} g Q}{4 h}(3-n) \\
& v_{d}=\frac{e^{2} g Q D}{2 h}=v_{+}-v_{-}
\end{aligned}
$$

Determiniag any two of the three NQR frequencles completely describes the magnttude of the EFG in the vicintty o. The nttrogen nucleus: 


$$
\begin{aligned}
e^{2} q \rho & =\frac{2}{3}\left(v_{+}+v_{-}\right) \\
\pi & =\frac{2\left(v_{+}-v_{-}\right)}{e^{2} g Q}
\end{aligned}
$$

As another example, for nuclet of $\operatorname{sptn} I=5 / 2$, such as aluminum-27, the solution of the Schroedinger equation can be shown to lead to a threeIevel system of energies(6) given by solutions of the secular equation(9)

$$
\varepsilon^{3}-7\left(3+\eta^{2}\right) E-20\left(1-\eta^{2}\right)=0
$$

wheie $E$ is in units of $\mathrm{e}^{2} \mathrm{qQ} / 20$. For the case, $n-0$, the energies of the three elgenstates are:

$$
\begin{aligned}
& E\left( \pm \frac{1}{2}\right)=-4 \frac{e^{2} g Q}{20} \\
& E\left( \pm \frac{3}{2}\right)=-1 \frac{e^{2} g Q}{20} \\
& E\left( \pm \frac{5}{2}\right)=+5 \frac{e^{2} g Q}{20}
\end{aligned}
$$

The frequencles of the resultant allowed transitions are given by:

$$
\begin{aligned}
& v_{1}-v\left(\frac{3}{2}+\frac{1}{2}\right)=\frac{3}{20} \frac{e^{2} q Q}{h} \\
& v_{2}=v\left(\frac{5}{2}+\frac{3}{2}\right)=\frac{6}{2} \frac{e^{2} q Q}{h}
\end{aligned}
$$

For the general case, $n \neq 0$, no solution in closed form cain be written down. However, tables of the results of numerical solution of the eigenvalue

(9)T. P. Das and E. L. Hahn, Nuclear Quadrupole Resonance Spectroscopy," Solid State Physics, Supplement 1, Acaderic Press, New York (1958). (Note that the error in the secular equation for spin $5 / 2$ given on page 13 has been corrected.) 
problem for $0 \leq \eta \leq 1$ in steps of .001 are ava1lable(10), so that observation of two NQR frequencles uniquely determines both the quadrupole coupling constant, $e^{2} q Q$, and the asymmetry parameter, $n$, of the site under study. For large values of $\Pi$, it becomes theoretically possible to observe the "forbidden" transition corresponding to the sum of $\nu_{1}$ and $\nu_{2}$ in Equation 10 . This may be especially useful to do in situations of low quadrupole coupling. As an additlonal example, for nuclel of $\operatorname{spln} I-7 / 2$, such as uranium-235, the solution of the Schroedinger equation can be shown to lead to a five-level system of energies given by solutions of the secular equation(5),

$$
E^{4}-42\left(1+\eta^{2} / 3\right) E^{2}-64\left(1-\eta^{2}\right) E+105\left(1+n^{2} / 3\right)^{2}=0
$$

where $E$ is in units of $\mathrm{e}^{2} \mathrm{qQ} / 28$.

For the general case, $n$ no solution in closed form can be written. However, tables of the results of numerical solution of the eigenvalue problem for $0 \leq \eta \leq 1$ in steps of .001 are also avallable(10), so that observation of two Identifled NQR frequenctes agatn uniquely determines both the quadrupole coupling constant, $e^{2} q Q$, and the asymmetry parameter, $\pi$, of the site under study. B. Experimental Techniques

Experimental techniques for the determination of NQR spectra can be divided into three categorles:

a. Cont1nuous-wave (CW) methods,

b. Transient wethods, and

c. Double resonsince techniques.

CW methods have enfoyed great popularity for they are simple and Inexpensive. The linitations of these techniques are so severe, however,

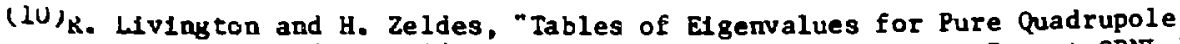
Spectra, Sp1n 5/2 and 7/2," Oak Ridge Rational Laboratory Report ORNL-1913 (1952). 
that in the low MHz region chey are rarely used anywore. Transfent methods include the superregenerative technlque (unproductive at the low frequencles of interest in many applied problems) and pulsed methods. Double regonance techniques are essentlally pulsed methods that monttor the resonance of one type of nuclear spectes while another is being perturbed. (7) When applicable, these methods are extremely sensitive and are very convenient for locating unknown resonances, although IIneshapes are not always rellable. Interested readers are referred to the excellent review by Edmonds. (11)

Pulsed techniques that operate in the so-called spln-echo mode are partlcularly good for applied NQR and they naturally lend themselves to remote detection. (12)

To understand the reasons for the advantage of the pulsed method $(13-17)$ it is necessary to begin by defining some basic magnitudes that describe an NQR Ifne. In the frequency domain an NQR line 1s ivlly described by 1 ts frequency and by a normalized shape function $S(\omega)$. For example:

$$
S(\omega)=\frac{2}{\pi \Delta \omega} \frac{1}{1+\left(\frac{\omega-\omega_{0}}{\Delta \omega}\right)^{2}}
$$

(II)D. T. Edmonds, Physics Reports 29, 233 (19,..

(12) T. Hirschfeld and S. M. Klainer, J. Mol. Struc. 58, 63 (1980).

(13) A. Zussman and S. Alexander, J. Chem. Phys, 49, 3792 (1968).

$(14)_{G}$. Petersen and T. Ofa, "Advances in Nuclear Quadrupole Resonance", - (J. A. S. Sm1th, Ed.) I, 179, Heyden, London (1974)

(15)Y. Abe, Y. Ohneda, M. B1rota and S. Koj1ma, J. Phys. Soc. Japan 37, 1061 (1974).

(16) A. Colligiani and R. Ambrosett1, Gazz. Chim. It. 106, 439 (1976).

(17) A. A. V. Gibson, R. Goc and T. A. Scott., J. Mag. Res. 24, 103 (1976). 
However, spin-echo techniques operate in the time domain; here there are three parameters that describe an NQR absorption line:

a. Spin-lattice relaxation time, $T_{1}$. The characteristic rime wth which a bulk magnetization is established and energy can flow between the spin system and the lattlce.

b. Spin-spin relaxation time, $T_{2}$. The characteristic time which describes the coupling between nuclet and which establishes the time scale for the observation of spin echoes.

c. A spin-echo shape function, $G(t)$. The "width" of this function is defined as $2 T_{2}^{*}$. $G(t)$ is the Fourier transform of the IIne-shape function, $S(\omega)$; thus $T_{2 \infty}^{*} \frac{1}{\Delta \omega}$.

Cont1nuous-wave $(\mathrm{CW})$ and superregenerative methods are poor techniques for lines with large $\mathrm{T}_{1}$ and/or large $\Delta \omega$ values (short $\mathrm{T}_{2}$ ) assoclated with the NQR IIne (to avold saturation in $\mathrm{CW}$, the Input power level has to be reduced to very small values). Broad Ifnes, expected to be Important in applied NQR, are weak since thefr areas are constant. Spln-echo wide-line methods are not only unaffected by long $T_{1}$ values, but since $G(t)$ and $S(\omega)$ are reizted by a Fourler transiormaiton, the equality

$$
G_{\text {uax }}=\int_{0}^{\infty} s(\omega) d \omega=1
$$

holds (Fig. 3). Thus, broad lines in the frequency domain do not affect the maximum intensity of the echo signal.

The NQR spectrum of a substance is determined by placing about 25 grams of sample inside the inductor of a tank c1rcult, which is then subjec:ed to a series of radio-frequency pulses of frequency $f$. Whenever the frequency of of these pulses satisfies the resonance condition $f=v_{Q}$, where $v_{Q}$ is one 
of the quadrupole fraquencies, absorption of exergy takes place and is retransmicred as a series of sigmals (free induction decay (FID) or spin ecnof. Therefore, by monitoring and detecting the transmitted signals as a iunction of the frequency of the pulses, the energy levels of the quadrupole nucleus are completely determined. A number of different pulse sequences are possible; this results in more or less eficient signal production, depending on the values of the relaxation times.

\section{NUR INSTRUMENTATYON AND DATA HANDLING}

For applied NUR spectroscopy pulsed techniques are used in conjunction with a variety of pulse sequences and FT data processing. This approach provides for maximum sensitivity and versatility.

\section{A. Pulsed NUR Spectrometer}

Tne most advanced pulsed NQK spectrometer in the nitrogen frequency region presently in operation was described by harding et al. (18) in 1979. A block didgraw of this instrumene is shown in Fig. 4. This is an F?-NQR spectrometer which operates from 0.5 to $5 \mathrm{MHz}$. The features of this instrunent are;

(a) The use of heterodyne techniques throughout to eliminate carrier feed througn.

(b) A matching network design that allowb i e-knob tuning of both the transmitter and receiver.

(c) The choice of several excitation sequences (Carr-Purcell, Meiboom-Gil\} modified $c: r-P u r c e l l$, spin-locked spin-echo and the standard sequence of $\pi / 2$ pulses) for generation and collec:ion of signals at high data rates.

(d) Fast Fourier transform roucines for studying line shapes, for

(18) J. C. Harding, D. A. Wade, R. A. Marino, E. G. Sauer and S. M. Klainer, J. Hag. Res. 36, 21 (1979). 
facilitating epectral searches, and for improved senaltivity.

(e) Ability to handle small samples $(0.1 \mathrm{cc})$. (19)

The specifications for th:s instrument are given in Table I, column 1 . Th1s instrument, although state-of-the-art and much more versatile and sensitive than previous spectrometers, is not optimally sulted to many applied NQR uses. A new system sulted for practlcal NQR use is presently under design and scheduled for construction in early 1981. It's anticipated specifications are shown in Table I, column 2. When this 1nstrument 18 operational, many of the obstacles whlch hinder NQR growth should be overcome.

\section{B. Pulse Sequences}

In order to optimize the NQR data format, it is necessary to choose the pulse sequence most sulted for the measurement. Several of these exist and each has 1tg benefits.

\section{1) Free Induction Decay}

The NQR response of a single crystal to a resonant pulse of rf Irradiation 19 completely analogous to the spln $1 / 2$ NMR case. One difference is that the Intensity of the free Induction decay (FID) response depends on the orlentation of the radio-frequency field $\mathrm{H}_{1}$ with respect to the EFG principal axes system. For a spin I - 1 system; the three resonance lines $v_{4}, v_{-}$and $v_{d}$ can be observed only when the field $\mathrm{H}_{1}$ is oriented, respectively, along the $x, y$, and $z$ principal axes of the EFG tensor. AB an example, for a $v_{-}$line, the expected value of the magnetization along the y axis 18 proportional to:

$$
\frac{\ln }{3 k T} \sin \left(\sqrt{2 \omega_{1}} t_{w}\right) \cos \left(2 \pi v_{-} t\right),
$$

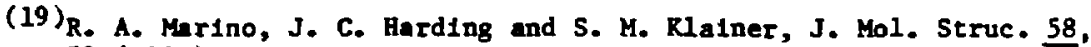
79 (1980). 
Iable I

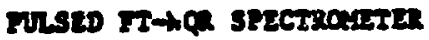

\section{Spac1fleartog}

Iraguaxey Mage

sanple Volue

Syerea Sensitiviey (SLSE) ${ }^{C}$

S/A - 3, 1 sec lntegration

Syoten Recovery IIse

(Sample Q-120)

$\pi / 2$ Pulse H1deh

Sapla Co11s

Kateblay lletworke

Sapple Operating Teuperature

Rewore Detect10n

Autometie Spectral Seareh
Extatine syates"

0.5 to 2014

40 to $0.1 e e^{b}$

$200=$

150 usec $341 \mathrm{k}$

$50 \mu \mathrm{sec}$

1 per extave

1 per oesave

$77^{\circ} \mathrm{x}$ to $350^{\circ} \mathrm{K}$

we, non-d1recesonel

Mo

\section{Sraten Dader Denten}

0.5 to 64 Matis

100 to $3 \times 10^{-6} \mathrm{ec}$

$1 \%$

$45 \mu \mathrm{sec} @ 1 \mathrm{MHz}$

$10 \mu \mathrm{sec}$

Sene

Seno

$4^{\circ} x$ to $350^{\circ} x$

be, directional

ye, programased equence
a) Bafarence
(18)
b) Beference
(19)
c) Baferance
(21) 
where $\omega_{1}=\gamma \mathrm{H}_{1}$ measures the intensity of the Irradiation, and $\tau_{w}$ is the duration of the 1rradiating pulse. The maximum response is obtalned when:

$$
\sqrt{2 \omega_{1} t_{1}}=\sqrt{2} \mathrm{H}_{1} t_{w}=\pi / 2
$$

in analogy with the NMR case.

In NQR, however, the sample usually consists of a polycrystalline powder. Then a convolution for all orientations must be ade. The result $(20)$ is that the $s 1 \mathrm{n}\left(\sqrt{2} \omega_{1} t_{\mathrm{w}}\right)$ function in the expression for the expectation value of the magnetization becomes a Bessel function, $J_{1}\left(\sqrt{2} w_{1} t_{w}\right)$. This function has its first maximum, analogous to a "90 pulse" at $\sqrt{2} \omega_{1} t_{w}=0.66 \pi$ and not at $0.5 \pi$ like the sine fynction. Siallarly, the first null, correspond1ng to a " $180^{\circ}$ pulse" occurs for a value of the argument equal to $1.43 \pi$ rather than simply to $\pi,(20)$

2) Spla Echo and Carr-Purcell (CP) Sequence

Following a FID experiment in a case where $T_{2}$ (spin-spin relaxation time) $>\mathrm{T}_{2}{ }^{*}$ (spin echo. shape function) it is possible to recall part of the magnet1zation not lost through $\mathrm{T}_{2}$ processes by applying a "180" pulse" at a time $\tau$ after the firgt pulse. As is well known, an echo will form at a time $2 \tau$ and this echo can be repeatedly recalled at integral multiples of this time $2 \mathrm{n} T$ by the application of additional " $180^{\circ}$ pulses" at times (2n-1)T. The amplitude of the resultant echo train decays with time constant $T_{2}$ (FIg. 5 ). S1gnal-to-nolse enhancement can be obtalned by coherently adding auccessive echoes in the sequence. The optinum time for co-adjition is easily shown to be $1.26 \mathrm{~T}_{2}$. Due to the generally amall value of $\mathrm{T}_{2}$ in solids, however, this nethod does not result in an appreciable enhancenent of the signal-to-nolise rat1o.

$(20)_{G}$. L. Fetersen, Ph.D. Thesis, Brown University (1975). 


\section{3) Spin-Lock Spin-Echo (SLSE)}

A wajor advance in $S / N$ enhancement was ade $b y$ Marino and klainer(21) when an adaptation of the Ostroff-Waugh sequence(22) was made to NQR. The sequence needed 18 essentlally a Kelbrom-G111-modified-CP sequence where all the pulses have the same fl1p angle of "90"-, wh1ch means $0.66 \pi$ in the NQR of an $I=1$ nucleus. In $\mathrm{F} 1 \mathrm{~g} .6\left(\mathrm{NaNO}_{2}\right.$ at $77^{\circ} \mathrm{K}$, on resonance) and $\mathrm{F} 1 \mathrm{~g} .7$ ( $\mathrm{NaNO}_{2}$ at $77 \mathrm{~K}, \mathrm{~s}$, Ightly off resonance) it can be seen that the spin echo train in this sequence persists for times of order $T_{1}$ (spln lattice relaxation time, actually $T_{1 \rho}$ ) and not the much shorter $T_{2}$. Coherent addition of the echoes in this case results in considerable enhancenent of the $S / N$, siace $T_{1} \gg T_{2}$ is the typical sicuation in these solids. Martno and Klainer showed that the optimum enhancement $180.64\left(T_{2 \varepsilon} / 2 \tau\right)^{1 / 2}$ where $T_{2 \varepsilon}$ is the effective decay constant of the echo train and $2 T 18$ the spacing between ecnoes, or equivalently the spacing bețeen pulses of the excltation sequence. It was further shown that the NQR effect was completely analogous to the spin $1 / 2$ MMR case discussed by Waugh(23) In that the decay constant $T_{2 \varepsilon}$ tends to $T_{1 p}$ as $\tau$ is reduced to values less than $T_{2}$. Furthermore, for Intermedlate values of $T_{2 E}$, this parameter is proportional to $\tau^{-5}$, again in analogy to MR. FIg. 8 showe this functional dependence for $\mathrm{NaNO}_{2}$ at $77^{\circ} \mathrm{K}$. Recently, Cantor and Wugh (24) have developed a theory to explatn the main features of this NQR effect using a model of a polgcrystalline solid with each altrogen site having one nearest aetghbour.

(21) R. A. Karino and S. H. Klatner, J. Chen. Phys. 67, 3388 (1977;.

$(22)_{E}$. W. Ostroff and J. S. Wugh, Phys. Bev. Letters, 16, 1097 (1966).

(23) J. S. Waugh, J. Mol. Spac. 35, 298 (1970).

(24) K. S. Cancor and J. S. Waugh, J. Chen. Phye. 13, 1054 (1980). 


\section{4) Strong off-Resonance Conb (SORC)}

Recently a new pulsed NQR experiment has been uned by one of the authors (RAM) which can have conoiderable advantages in enhancing the $S / N$ ratio,of weak 11nes. This represents new and yet unpublished information.

The steady state response of an ensemble of nuclear spins, I = 1/2, In high megnetic field $H_{0}$ to a strong radto-frequency field $H_{1}$, applied off resonance by $\Delta f$, has long been known $(7)$. When all the conditions for the establishment of a.spin temperature in the rotating frame are met(7), the $x$-component of the magnetization, which 18 experimentally observable, is given by the expression:

$$
M_{x}=M_{0}\left[\frac{H_{1}(2 \pi \Delta f / \gamma)}{B_{1}^{2}+B_{10 c}^{2}+(2 \pi \Delta f / \gamma)^{2}}\right]
$$

where $H_{0}$ is the equilibrium longttudinal magnetization, $\gamma$ is the magnetogyric ratio of the nucleus, and $H_{10 c}$ is a measure of the local field at the nuclear site due to 1ts nelghbors. Results earogous to Eqn. 18 have also been derived and observed for a quadrupolar system(25) with nuclear spin I $s / 2$ when subjected to the same strong, long, off-resonant 1rradiation $H_{1}$.

The preliminary results obtained, when the frradiation field $H_{1}$ 1s applied in a long train of equally-spaced Identical pulses, are presented here. Although the SORC experinental data reported here are for a quadrupolar I - 1 system, analogous effects in a magnet1c system or a quadrupolar system wh spin differeat from unity can be expected.

F1g. 9a defines the paraneters of the SORC sequence. Here a train of radio-frequency pulses of duration $t_{w}$ and apacing $T$ ia applied, $\Delta f$

(25) J. C. Pratt, P. Kaghunathan and C. A. KcDowell, J. Chen. Phys. 61, 1016 (1974) and J. Mag. Res. 20, 313 (1975). 
amy frod exact resonence, to a pure nuclear electric quedrupole syeted ir. zero external magnetc field.

The variation of the signal emplitude ye. $\Delta f$, the distance from exact resonance, shows two features as deplcted in F1gs. 9b and 9c. Fsrst, the elgnel amplitude is aodulated by a unusold of period $1 / \tau$, the pulse repetition rate. This phenomenon 1s best underetood by considering that the Fourler transform of the transmitter pulses hes periodicity $1 / \tau$. This leads to successive onxima and wining in the NQR signal (F1gs. 9b and c) as che transoltter frequency is chenged, 1.e., $\Delta f$ is varied. Alternctively, and more natvely, this modulation can be interpreted as the destructive interference of type I sgaals (1mmediately following the if pulac) and type II signals (immediately preceeding the rf pulse) In thelr overlap region as the frequancy, $\Delta f$, is alowly varied.

Another feature shown In EIg. 9c is the shape of the envelope, possibly conforming to a function of the type $\Delta f /\left(A^{2}+\Delta f^{2}\right)$, such as Eqn. 18 . Insufficlent data have been taken so far to ascertain the degree of agreenent with theory on this last point.

The muclear Induction signals present in the observation window between succesetve pulses of the SORC sequnce are shown In F1ge. 10s-j as a function of the pulse separation $\tau$. All data were taken on the $V_{-}$line of $\mathrm{NeNO}_{2}$ at $77^{\circ} \mathrm{K}$. The angnitude of the type I signal is then plotted ve. $\tau$ In Figure 11. Wote that for $\rightarrow>B$ asec the angitude of type I signals increased with $T$ 28 wight be expected for a FID signal subject to epin-lattice relaxation. On the other band, for $\tau \leqslant 5$ neec, elgnale at both ends of the obsertution window are of a coaparable size and they grow exponentially with decreasing $\tau$. Th1s is the region of interest. 
F1g. 12 shows the varietion of the type II algnal 쁘. $\left\langle\mathrm{H}_{1}\right\rangle$ for $\tau=3$ mec. The experinental points are obtained for four different values of the Instantaneous field $H_{1}$ obtained by changing the pulse width $t_{w}$ at constänt "flip angle" $\sqrt{2} \gamma t_{w} H_{1}$. The dotted line 1s the curve $F=\left\langle\mathrm{H}_{1}\right\rangle /\left[\left\langle\mathrm{H}_{1}\right\rangle^{2}+\mathrm{B}^{2}\right]$ wth $B=0.05 G$. The fact that $\left\langle H_{1}\right\rangle$, the average value, rather than $\dot{H}_{1}$, the peak value, is the important parameter and that there is good agreement of the data with the form of Eqn. 1818 strong evidence that the ensemble of spins is responding to the cime-average field of the SORC sequence Ir a manner analogous to the conventional long, strong, off-resonant pulse.

The size of the paraneter $B 18$ found to be about 0.056 which 18 approximetely two orders of magnitude too small for the value expected from the contribution of $\Delta f$ to Eqn. 18. Th1s discrepancy 1 s reduced by a factor of 5 when the experiment is repeated for $\tau=1 \mathrm{msec}$, as shown in F1g. 13. ComparIson of Figs. 12 and 13 suggest that the pulse nature of the experiment is st1ll very important for $?=1 \mathrm{msec}$ and that quantitative agreement cannot be expected until $\tau$ is reduced further.

The potential of this techntque appears to be great since sigrals can be obtalned at essentially $100 \%$ duty cycle. However, further experimentation 18 needed to coupletely understand the operational parameters of SORC. C. FT Considerations

There are three maln reasons for doing FT spectroscopy:

a. Enchancenent of signal-to-nolse over CW nethods is given by the square root of the ratio of the total width of the opectrum to the typical line width, $\sqrt{T_{2}^{\star} \Delta \text {. }}$

b. Pulsed nethods are singularly well-sulted to deta processing.

c. The line shape 1s readily obtalned directly fron the output. 
Of these, the f1rst reason, probably the sost important in other disciplines, 1s not aearly as important in NQR, because the ratlo defined above 1a not large and can often be close to unity. This is so because in solids line widths are relatively large (a few $\mathrm{kHz}$ ) while the bandwidtho which can be sultably 1rradiated are in the $10-100 \mathrm{kFz}$ range. The other two advantages, howerer, have provided the impetus toward the growth of FT-NQR.

(1) Comparison with High Resolution MMR (HR-NMR)

In HR-MMR the width of the spectrum $\Delta$ is wuch less than the carrier frequency $f_{o} ; \Delta / f_{0}<10^{-3}$ can be expected even in worst cases. In NQR however, the ratio $\Delta / f_{0}$ is ofteriof order unity.

This Implies that the NQR spectrum must be obtained one frequency Interval at a time and cannot usually be displayed in one operation as in the case in HR-NMR.

2. Processing Techniques

Once FID or echo signals have been obtalned, the proper FT treatment for each w11 yield the desired lineshape spectrim. $(26,27)$ In this section the procedures the authors found most antisfactory are discussed.

The problen of phase correction for NQR pectra preaents a particular problew not found in fixed-frequency spectroweters. The fact that the NQR spectroneter operates at variable frequencles nens that instrumental phese shifts will be present for WQR signale which are not corrected for before final data collection. Theae phase ahifto can be conolderable and are of course also present in echoes, where the receiver dead tine proble present for FIV signals does not occur. In Table II the athenatical results

(26) R. Lenk and E. A. C. Lucken, Pure Applied Chen. 40, 199 (1974). (27) A. Coll1g1an1 and R. Abrosett, Gaz2. Ch1. Itallana 106, 439 (1976). 
for Lorentzian lineshapes are collected as guides to the solution of this problem.

The results in the Table show that the presence of phase shift.s produces an admixture of absorption and dispersion modes in the case of FID signals, and a poealble 1088 of intenesty in echo algals. In1s probles can be avoided while at the ame tine the true Lorentzian line ahape is preserved, if the modulue aquared transform is computed for FID signals, and the godulus tranaform is computed for echoes. Thus the proper line shapes are obtained in each case regardless of the derree and source of phese shift whout need for a separate "phese correction" eubroutine.

In F1ge. 14-21 the results of conputer-sinulated apectra are show. An echo and a FID signal have been aimulated for both Lorantzian and Gaussian Iineshapes. Coelae, sne, the square of the sodulus, and nodulus transforms are computed and diaplayed. F1gs. 14-17 are for no phase ahtft, while Figs. 18-21 gave a phase shift of $30^{\circ}$ in the tine dowaln signals. Note that in all cases the concluatone discused for proper data processing are borne out, anialy the the godulus gquered transform should be uned for FIb elgnale, and the aodulue trancform should be ued for echo signals.

F1ge. 22-24 show experinentel apectra that demonstrete the foregoing argunents. Fige, 22(a) and $23(a)$ are the NItrogen-14 NQR FID signals at $77^{\circ} \mathrm{K}$ from hexeethylenetetranine (HAT) and urea, respectively. The cosine and sine

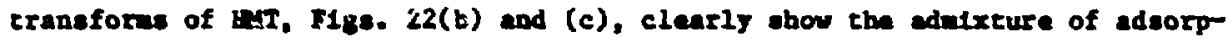
tion and dispersion expected when phese shifts exist in the time-donein data. This effect is wuch less evident in the cosine and alne tranaforne of urea, F1ys. 23(b) and (c), which occurred wth only a sall phace abift. Finally. the proper 110eshapes are shoma in Fise. 22(d) and $23(d)$, the codulue gquared 
TurE II

Lorentzien IID and echo complex elpale and thetr Fourter traneforms IID

IIme deanes: $f(\varepsilon) \cdot e^{-\alpha t} e^{1 \omega_{0} t} e^{-1 \phi} \quad \varepsilon \geqslant 0$

$$
\begin{aligned}
& \text { c, costee Eransform }-\frac{1}{a^{2}+\left(\omega-\omega_{0}\right)^{2}}\left\{\operatorname{acos} \phi-\left(\omega-\omega_{0}\right) \ln \phi\right\} \\
& \text { s, s10e eranoforo }=\frac{1}{\alpha^{2}+\left(\omega-\omega_{0}\right)^{2}}\left\{\alpha \sin \phi+\left(\omega-\omega_{0}\right) \cos \phi\right\} \\
& c^{2}+s^{2} \text {, Modulus squared erenefors }=\frac{1}{a^{2}+\left(\omega-4_{b}\right)^{2}} \\
& \left(c^{2}+s^{2}\right]^{2} \text {, Modulus transform }=\left[\frac{1}{a^{2}+\left(w-w_{b}\right)^{2}}\right]^{\frac{1}{2}}
\end{aligned}
$$

\section{0}

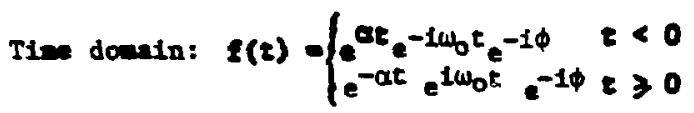

$$
\begin{aligned}
& \text { c. costas transfore }-\frac{1}{a^{2}+\left(\omega-\omega_{0}\right)^{2}} 200004 \\
& \text { 3. ston tranfore }=\frac{1}{a^{2}+\left(\omega-\omega_{0}\right)^{2}} 20010
\end{aligned}
$$

$c^{2}+s^{2}$, Hodulus equared traneform $-\left[\frac{2 a}{a^{2}+\left(w-w_{b}\right)^{2}}\right]^{2}$

$$
\left[c^{2}+s^{2}\right]^{3}, \text { nodulue errasefore - }\left[\frac{2 a}{a^{2}+\left(\omega-\omega_{0}\right)}\right]
$$


transforms of the time-dornaln signals. Note the fine structure on the mit 11ne, firgt reported by Colliglanl and Ambrosett1.(28) The modulus transforms, Figs. 22(e) and 23(e), are shown for comparison and they are visibly broader than the true I1neshapes.

An example of an echo signal is shown in FIg. 24(a), a doublet of $v_{-}$ IInes from the monoclinic phase of TNT at $77^{\circ} \mathrm{K}$. FIgs. 24(b) and (c) are, respectively, the cosine and sine transforms of this signal. Note that, as expected, both of these transforms yleld valid lineshapes with fractional amplitudes, and that the modulus transform, Hig. $24(\mathrm{e})$, ylelds the correct IIneshape.

\section{IY. APPLICATIONS}

The current intense interest in the chemistry, physics, and crystiolography of solids is responsible for the renewed activity in NQR. In 1964 Grechishkin and Solfer(29) suggested spectflc applied categorles Into which NQR could be divided. These have been updated and include:

a. Investigation of the nature of chemical bonds in solids. (The NQR frequency is directly dependent upon the type of hybridization and the degree of lonization of the chemical bond.)

b. Establishent of nonequivalence of the location of resonant atons in crystal lattices and molecules.

c. Wualitative analyses (each chemical compound has a definite NQR spectrum).

d. Inspection of puricy of chenical synthesis products (The NQR IIne

(28)A. Colligiani and R. Abrosert1, J. Chew. Phys. 60, 1871 (1974).

(29)V. S. Grechishkin and G. D. Solfer, Pribory 1 Tekhnika Eksperimenta 1, j. (1964) (Rusaian). 


$$
\text { A-23 }
$$

Intensity is directly dependent on the mount of soluble impurities In a sample.)

e. Structural analyels of cryetals. (NQR 1a a valuable complimènc to x-ray methods.)

f. Detection of phese tranaftion in crystals and the investigation of their kinetice.

8. Heasurewent of average rotary vibration frequencies and average monents of Inertia of molecules in cystals, from the temperature dependence of NQR IInes.

h. Masurcient of atrain.

1. Determination of tenperature.

Table III liets sone of the capabilities of NQR. These eximplen have been chosen because they represent real, current-day problens which heve been presented for possible solutior using NQR. 
Table III

\section{Sugrested Applications of FT-NQR*}

Characterization of new compounde (drugs, chemicale, explosives, 1:quis crystals, etc.).

vegree of cryetal order (in clays, solid solutions, etc.). Locus of aluminum atons in plagioclase minerals.

Shelf life of waterials (drugs, chemicals, explosives, etc.). Determination of in-situ strain (salt dooes, hard rock, etc.). Detection of contraband (drugs, explosives). Identification of contaminants (1.e. nature of $N, S$ and 0 in coal). Measurement of temperature. Kadiation damage In solids, Crystalline polyworphim sudies (1.e. relationship of TNT crystalline forme to impact atability, etc.). Measurenent of organic conplexes (1.e. $\mathrm{V}, \mathrm{Cu}, \mathrm{Ni}$ in crude oll). Identification of host minerals in bore-holes (1.e. measurement of uranium grade and type).

Characterization of phase trangitions (1.e. In rock-forming minerals auch as feldspars, pyroxenes, phyllosilisates, etc.). Interpretation of crybtal order with respect to thermodynamic properties (in ninerals).

Deterwination of non-stolchionetric behnvior (1.e. minerals, alloys single crystals, etc.).

Idencification of enall solid samples (i.e. exsolution in aluminofllicates, dangerous aterlala, rare compounds, etc.).

In sone instances NQR techn1ques other than FT may prove to be more suttable, i.e., double resonance NQR, acoustle NQR, etc. 
y. CoNchusions

It 1s now possible to anweer the two questions poeed in the introduction. Nuk developent hes been hindered by:

(a) Lack of proper inetrunentation

(b) Inefficient saple excitation technique:

(c) Primitive data handing and proceseing aethods.

It can be shown that solutions havo been found for each of these drawbacks:

(a) Sensitive, higì resolution, autonated, pulsed NQR spectroweters which ut1lize state-of-the-art electronlc, radar, and computer techniques fulfill the instrumentation needs.

(b) The availability and usabllity of variety of pulse sequences such as spin-Jock spia-echo (SLSE) or strong off-resonance comb (SORC) provide superiox sample excitation.

(c) Fourier Transform data processing, using modulus squarad or modulus transforms, represents the state-of-the-art procedure for the spec1al NQR conditions.

The need for FT-NQR is clearly demonstrated by the examples given in Table III. The growing 1nterest in understanding the behavior of solids has provided an impetus for analyical techniques sultable for use with solld state gystens. Particular anphasis has been directed at antural systems, such as ninerals, and wilitary and security applications. Furthermore, the poselbility of measuring ig-eltu atrain is of enjor 1nterest to the rock nechenico comunity. A technology which can wesure both single cryetals and polycrystalline ateriale, which can respond to contaninants or homogeneous mall sanplea, which can operate both in the laboratory or under seat-renote conditions whlle providing high resolution spectral data for solid saples has 


$$
\text { A-26. }
$$

al of the veratility to develop into an inportant analytical athod.

The authore wh to thank Mr. R. Comore of Hlock Engineering, Cambridge, $M A$ for his assistance in runing the $\mathrm{KQR}$ spectra. They would also like to recognizz Mr. N. Henderson, Office of Nuclear Wate Isolation, BattelleColubux, Columbus, OH and Dr. C. Boghosian, U. S. Army Research office, Reaearch Triangle Park, N.C. for their continued support. Technical editing was done by Dr. Ellory Schemp of Lawrence Berkeley Laboratory, Berkeley, California. 


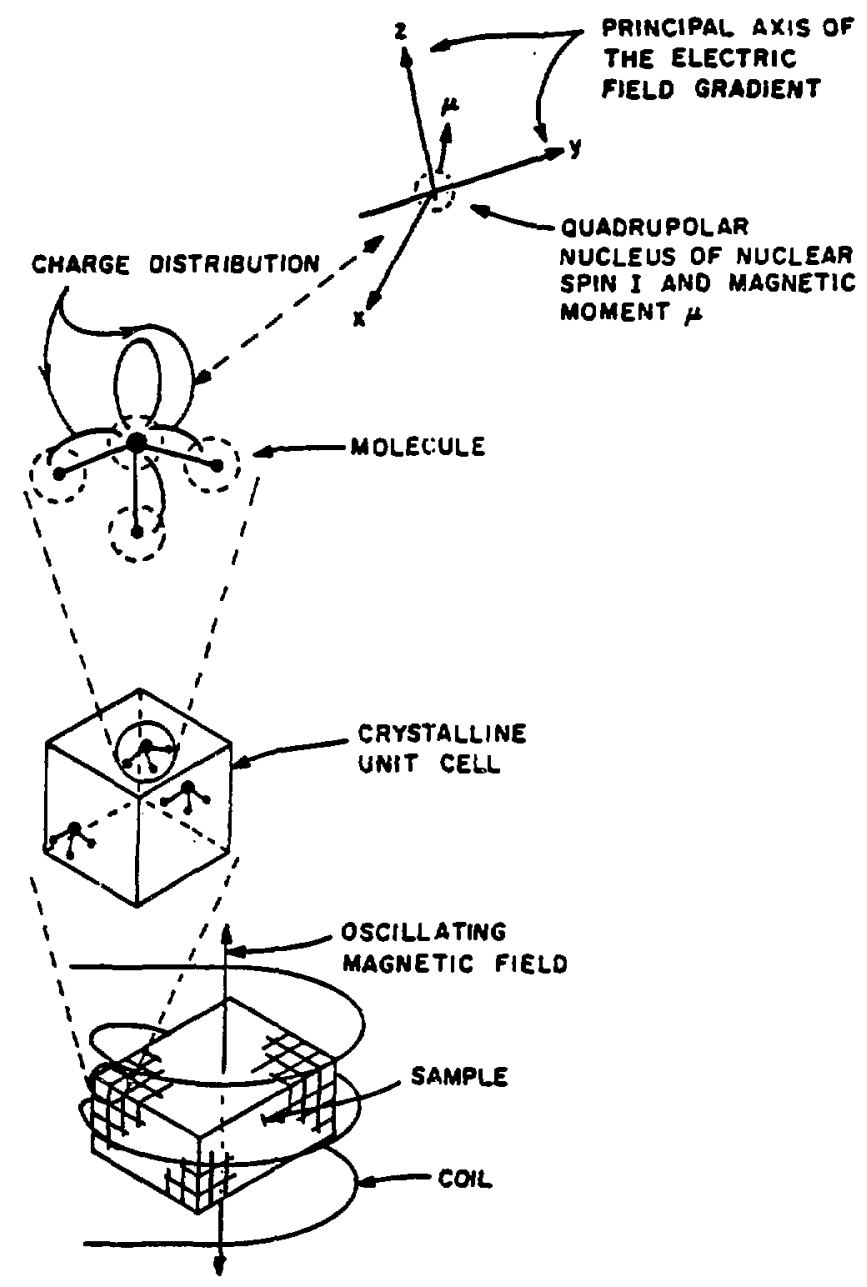

\section{Figure I. Graphic Display for Nuclear Quadrupole Resonances.}

XBL 793-8926A 


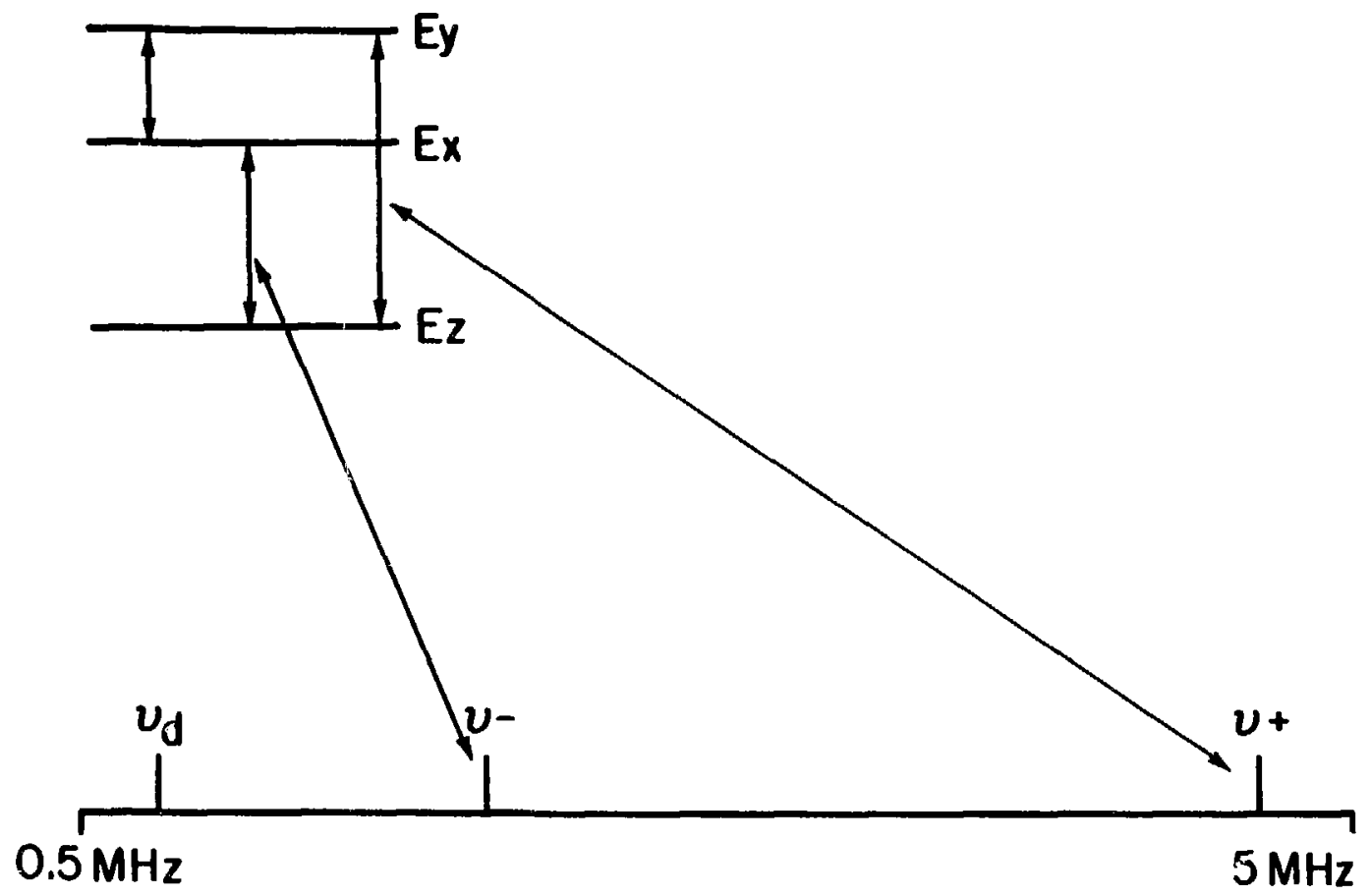

Figure 2. Energy Level Diagram and Approximate Frequency Range for Nitrogen-14 NQR. 
FREQUENCY DOMAIN

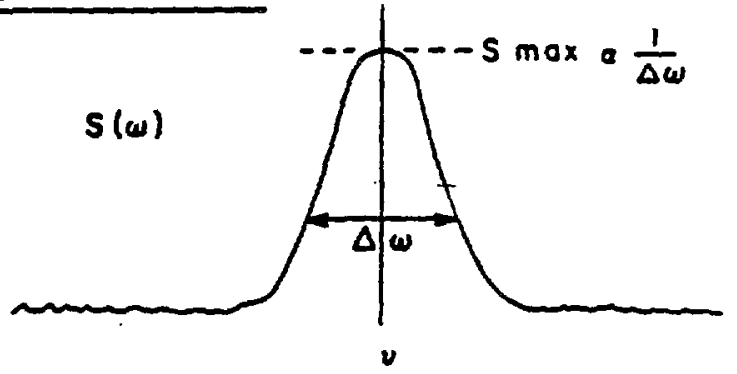

TIME DOMAIN

FID

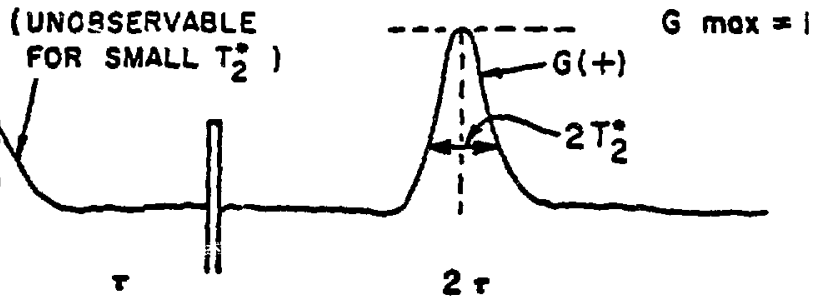

Figure 3. Line Shape Porameters for Spin Echo Sequence.

XBL 808 -2701A 


$$
\text { A-30 }
$$

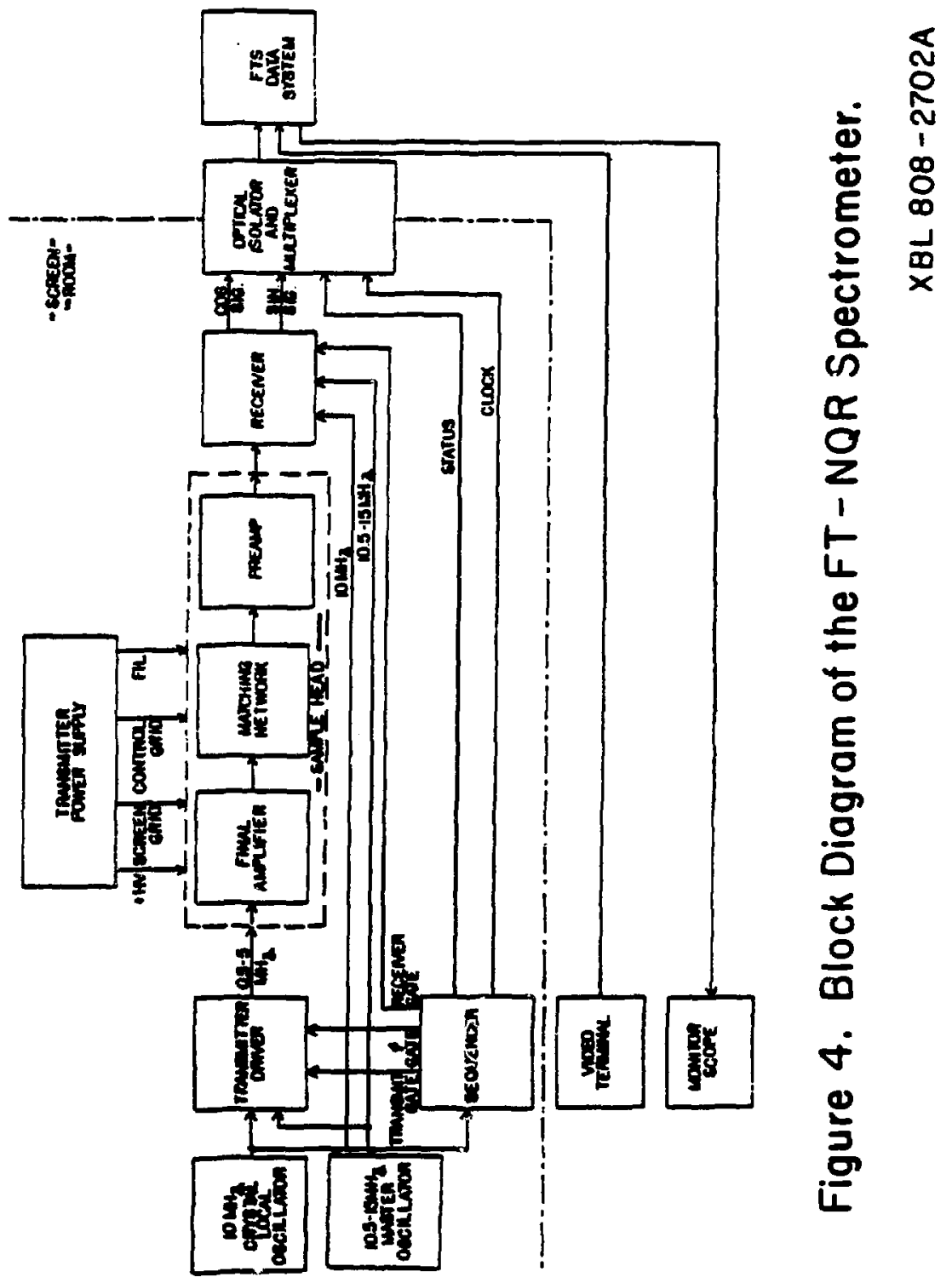



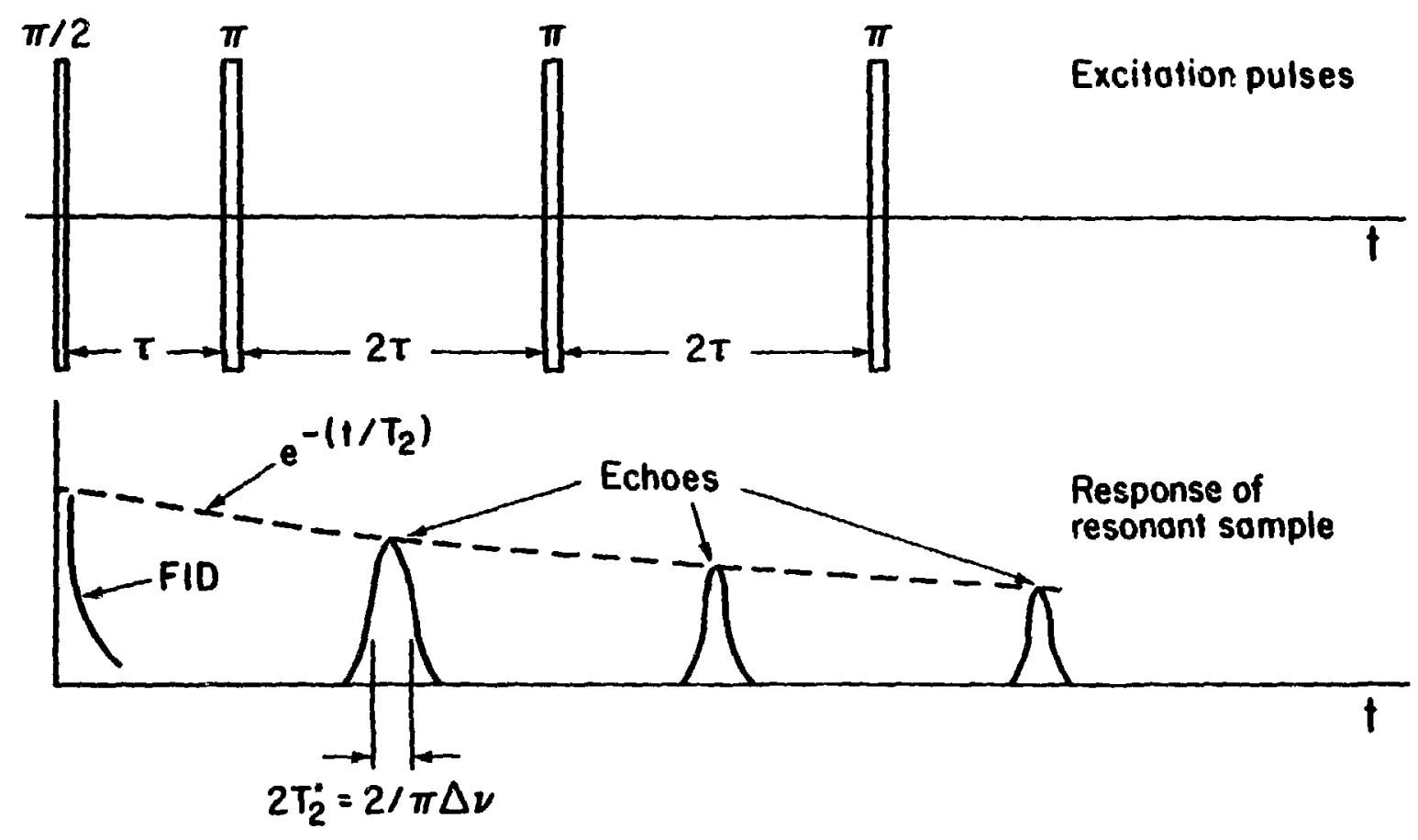

Figure 5. Parameters for the Carr-Purcell Sequence. 


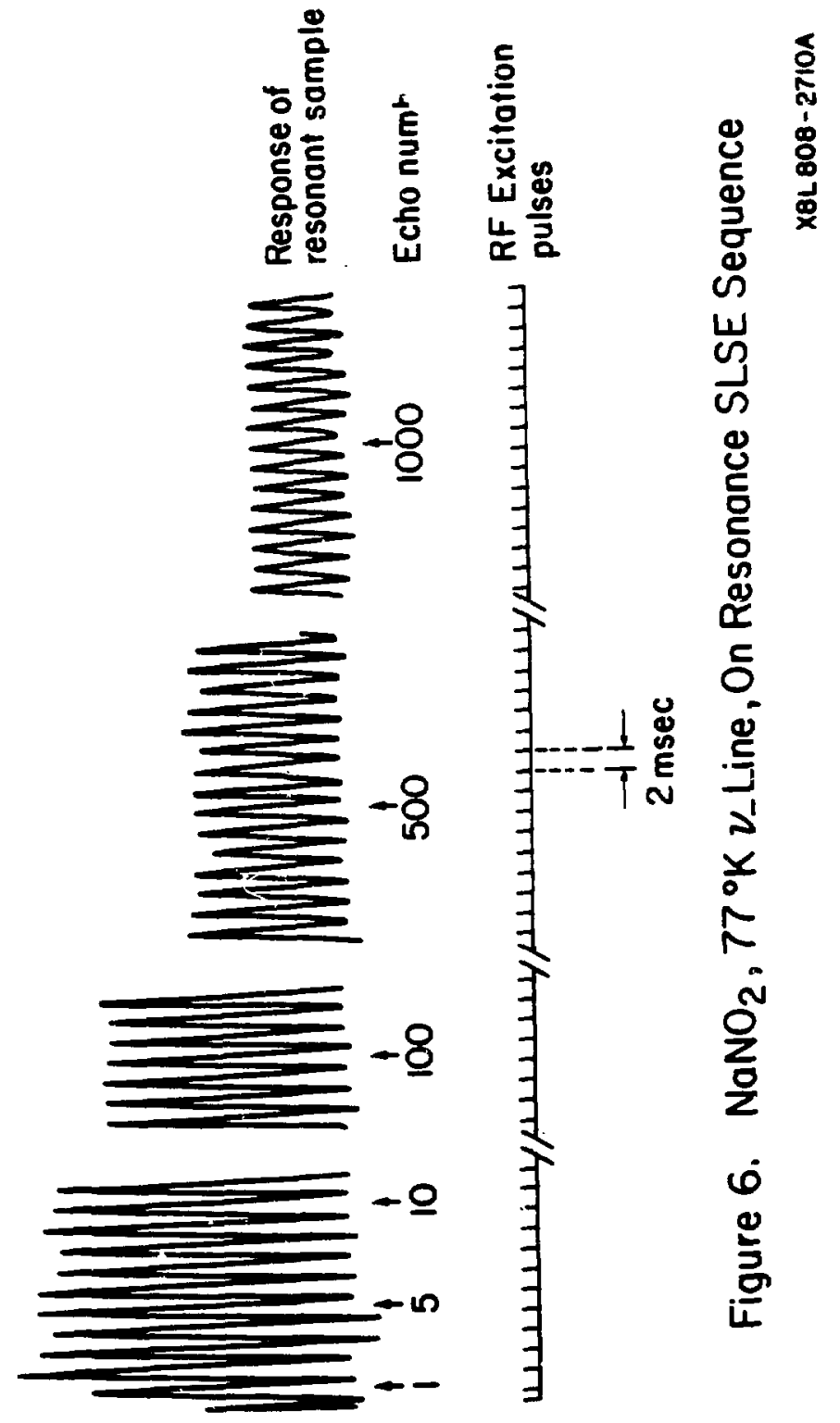




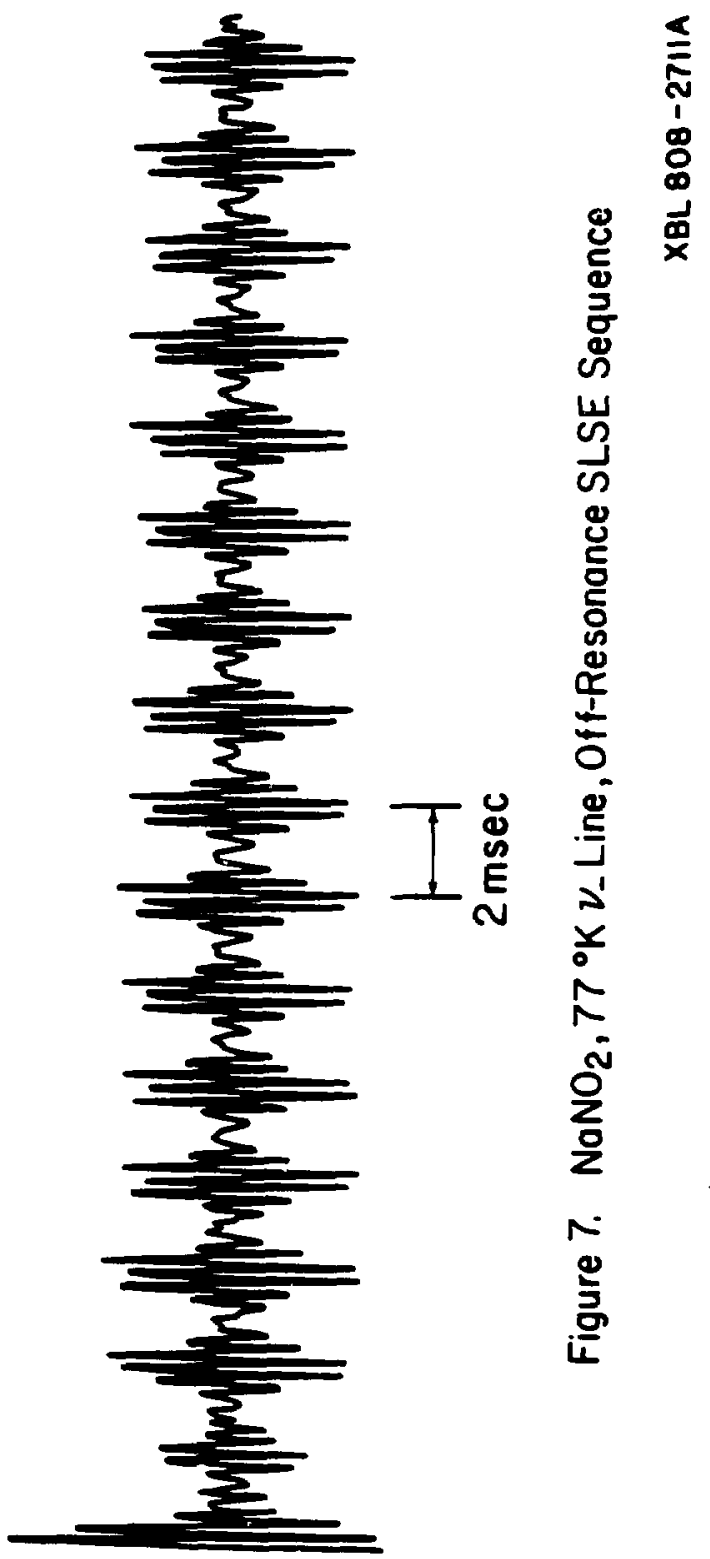




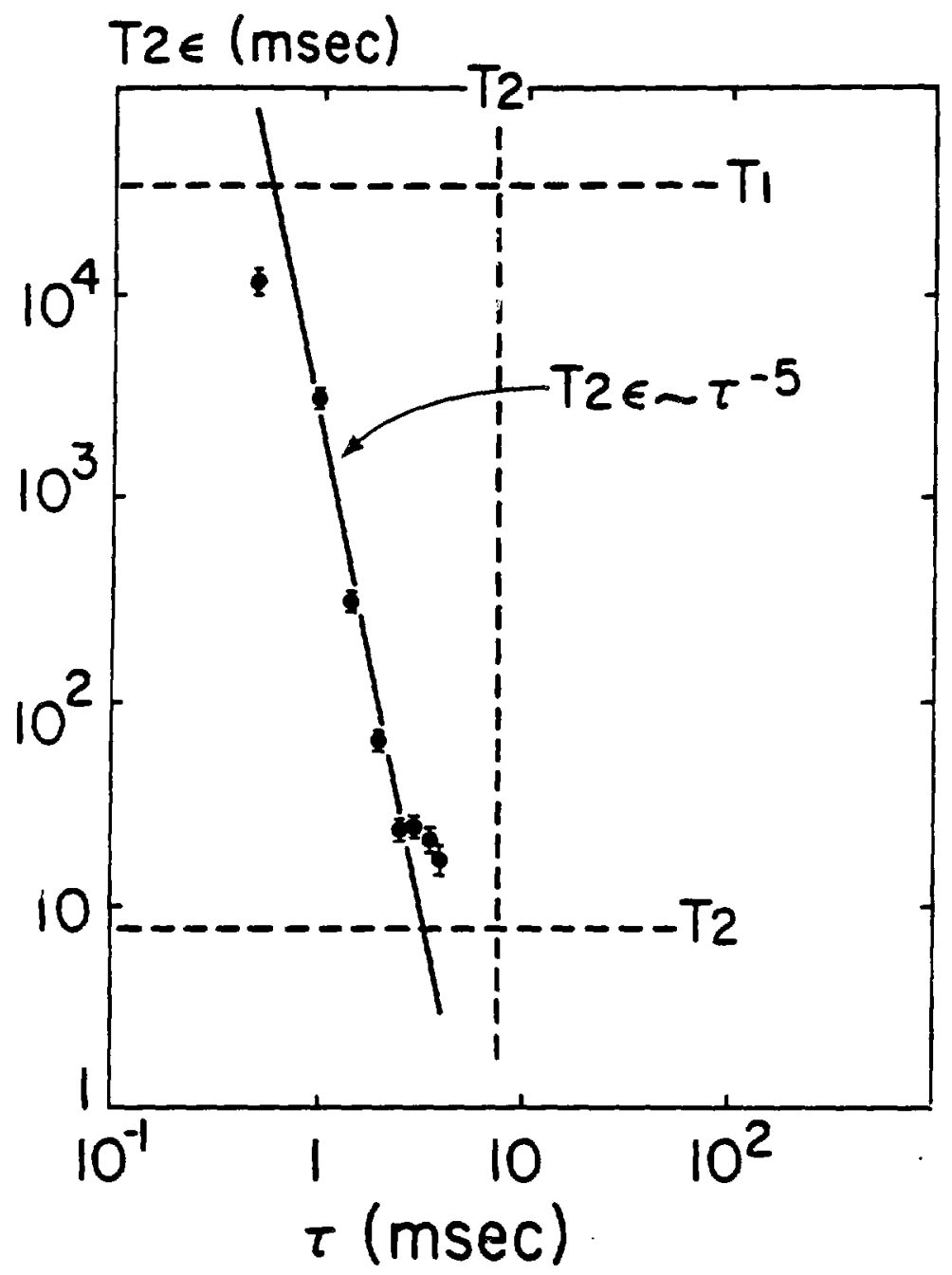

Figure 8. Echo Train Decay Constant, T2e vs. SLSE Pulse Spacing $\mathrm{r}$ for the $\nu_{-}$Line of $\mathrm{NaNO}_{2}$ af $77^{\circ} \mathrm{K}$ 

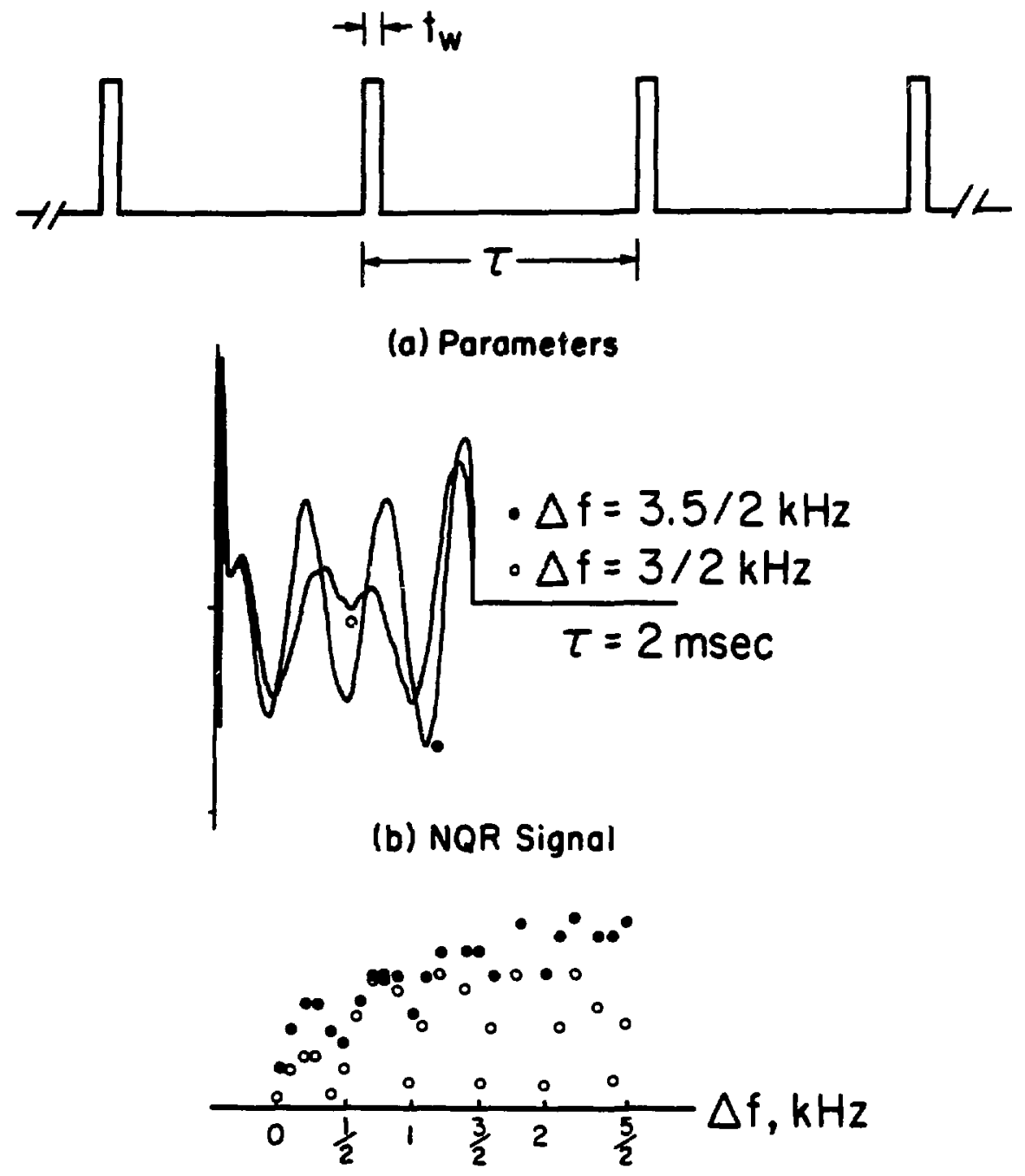

(c) NOR Signal vs. Resonance Offset

Figure 9. SORC Pulse Sequence 


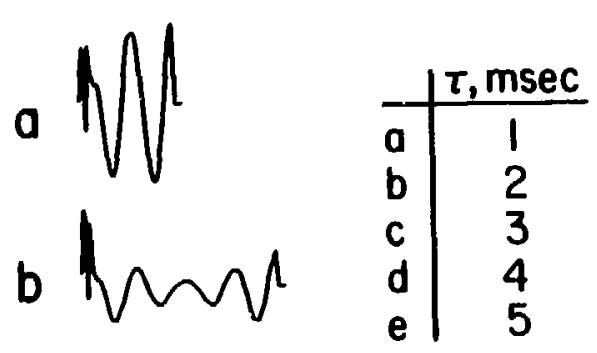

chn
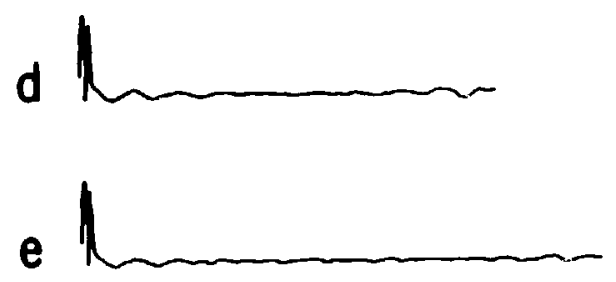
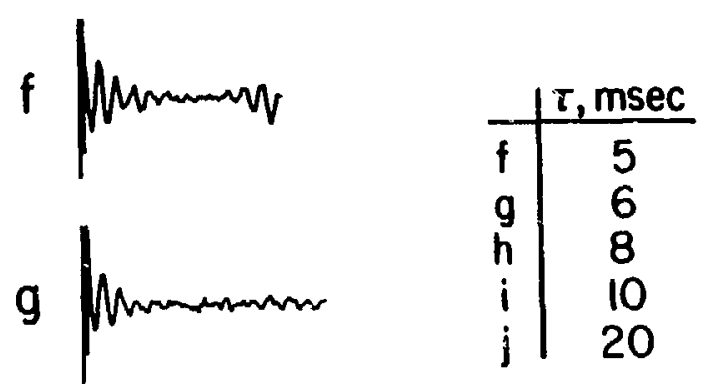

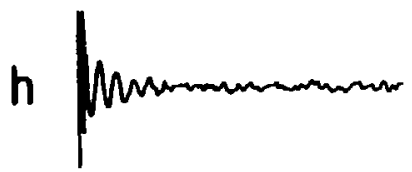

rigure 10. Steady State SORC Signal vs. $\tau$ 


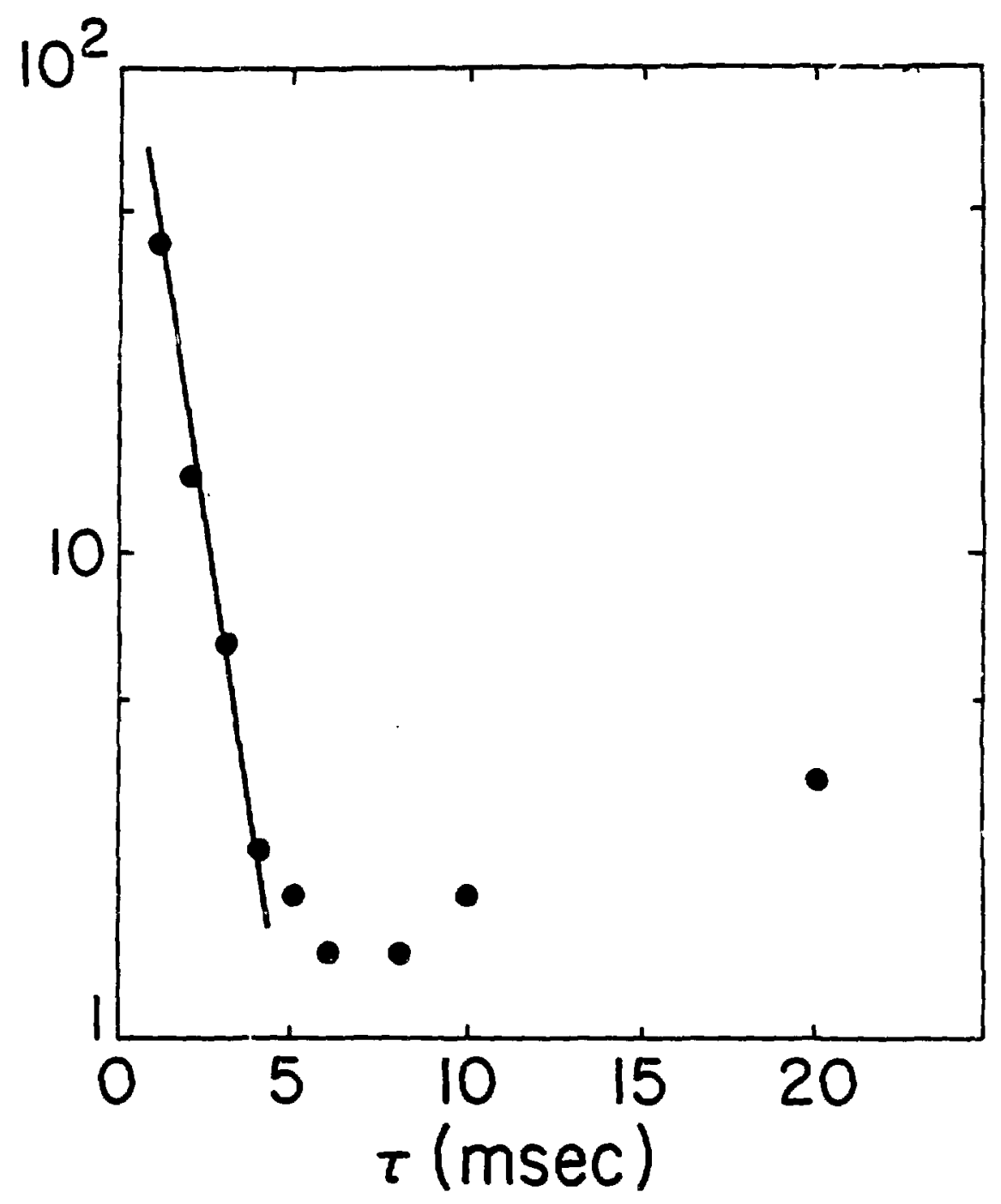

Figure 11. Semilog Plot of SORC Signal vs. $\tau$ XBL 508-2707A 


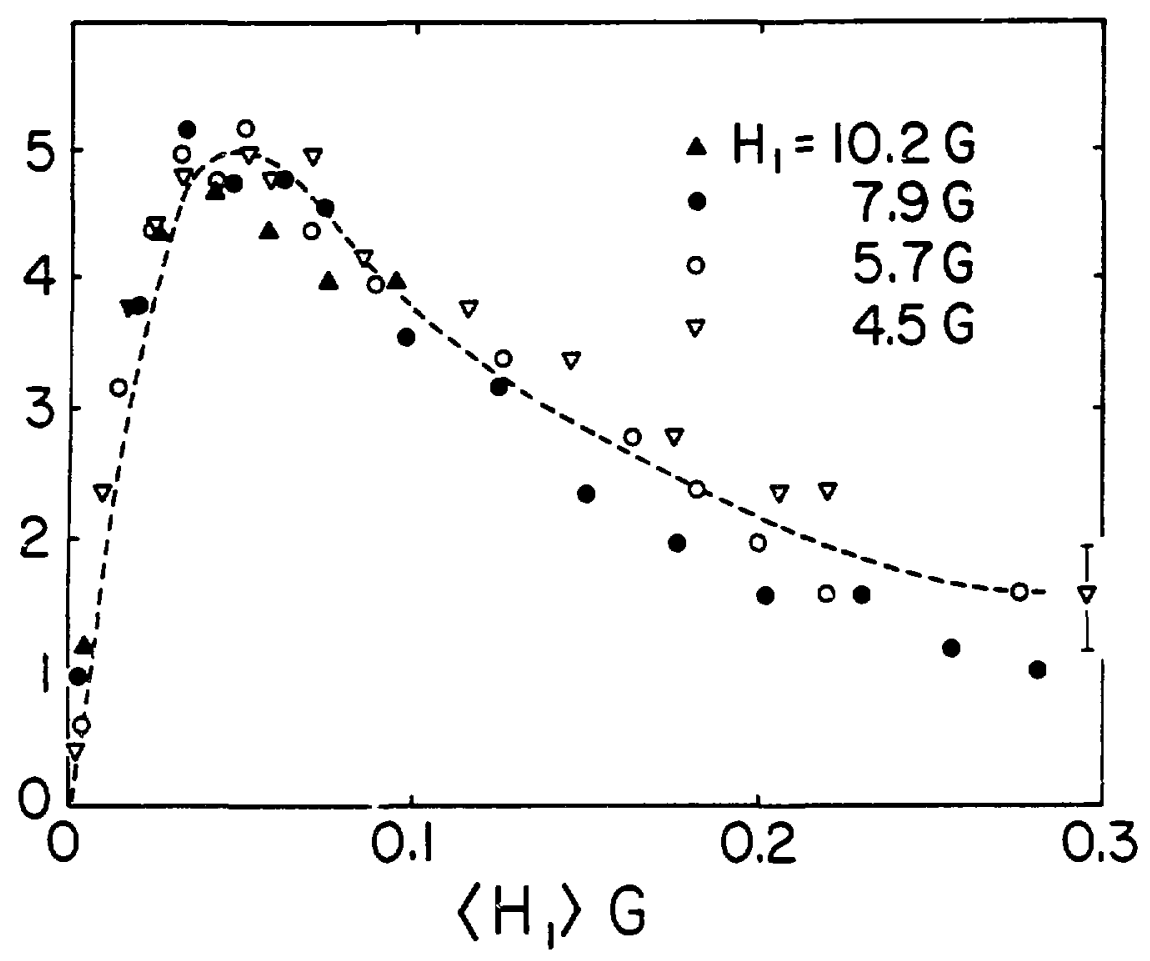

Figure 12. Size of SORC Signal vs. Average Value of $H_{1}(\tau=3 \mathrm{msec})$

XBL 808 - 2708A 


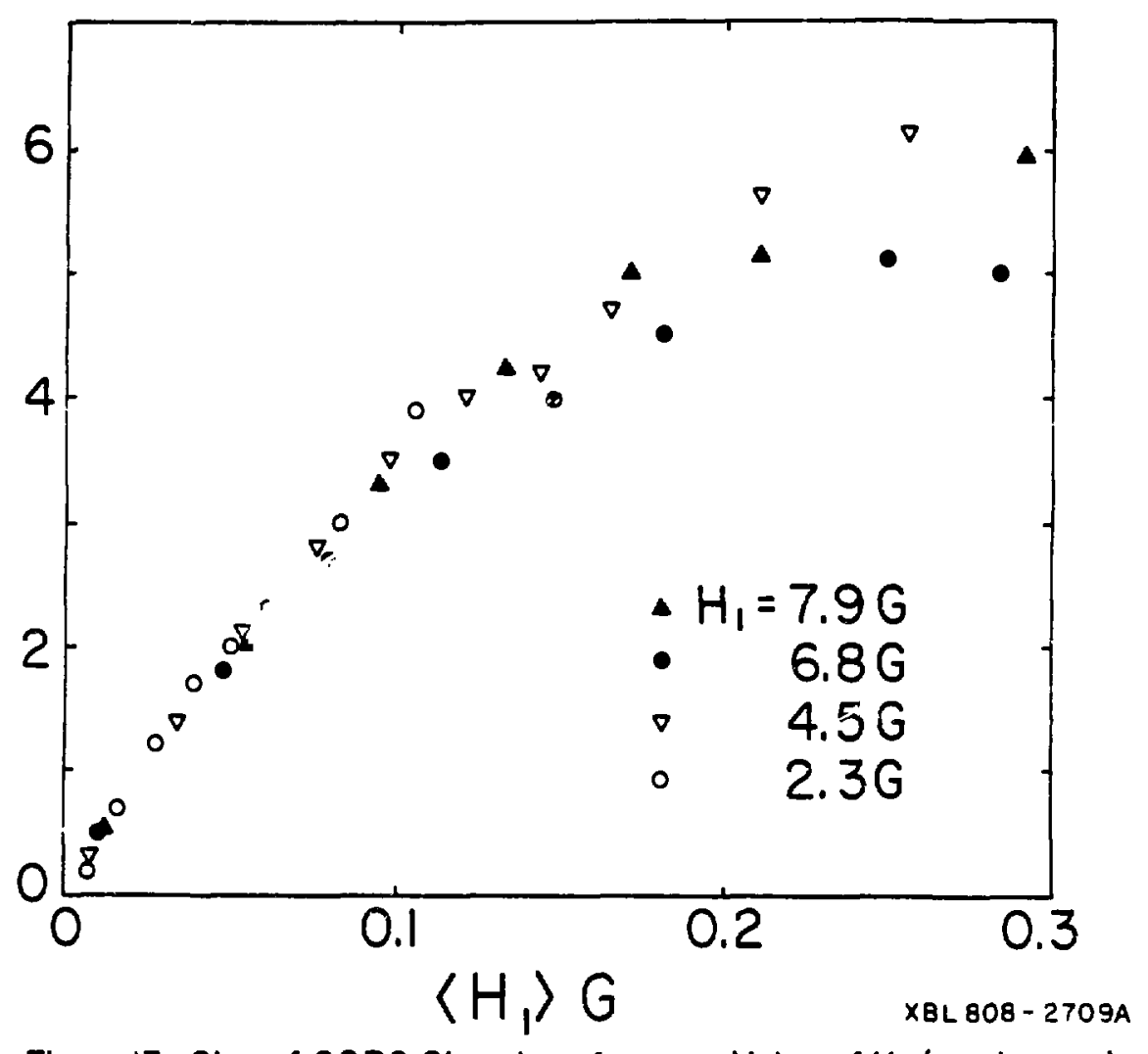

Figure 13. Size of SORC Signal vs. Average Value of $H_{1}(\tau=1 \mathrm{msec})$ 


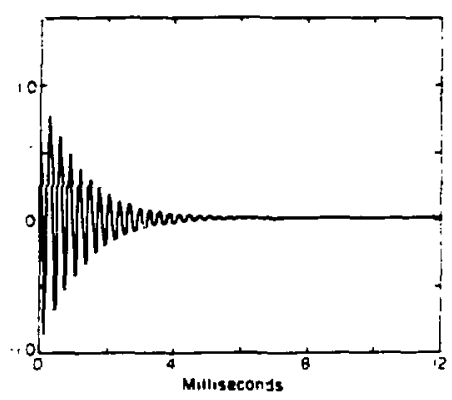

(a) Exponentrol $F I 0, \phi=0$

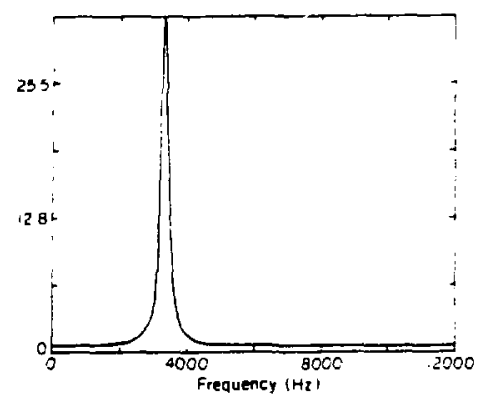

(b) Cosine FT

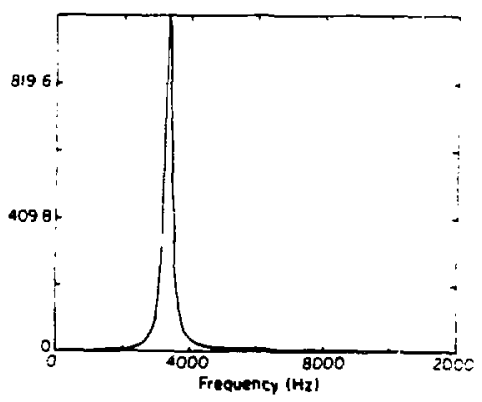

(d) Modulus Squored FT

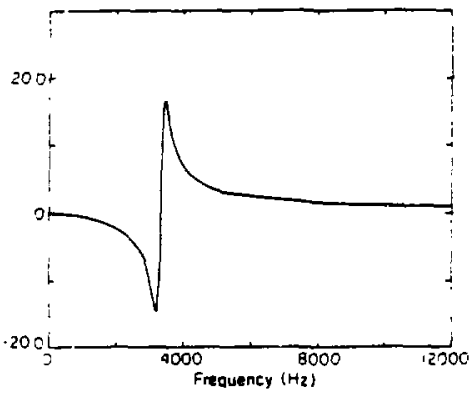

(c) Sine $F$

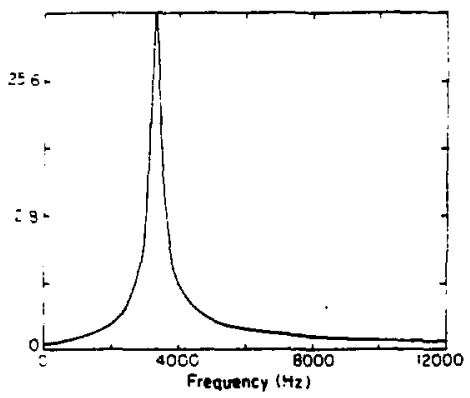

(e) Modulus FT

$\times 6 L, 806-27208$

Figure 14. Simulated Exponential FID $(\phi=0)$ and its Fourier Transforms 


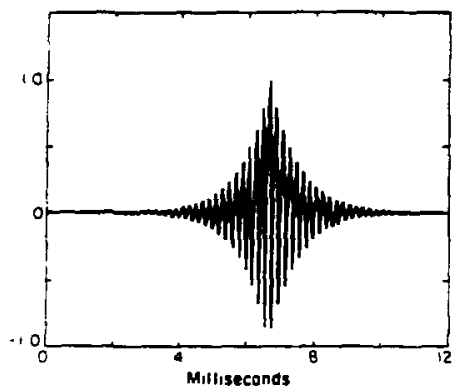

(o) Exponentiol Echo, $\phi=0$

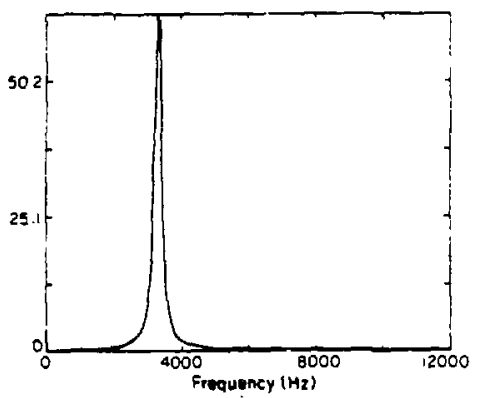

(b) Cosine FT

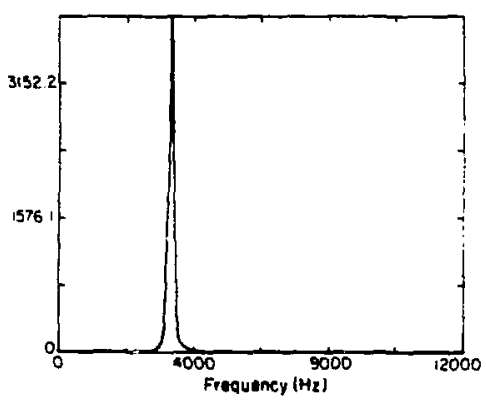

(d) Modulus Squored FT

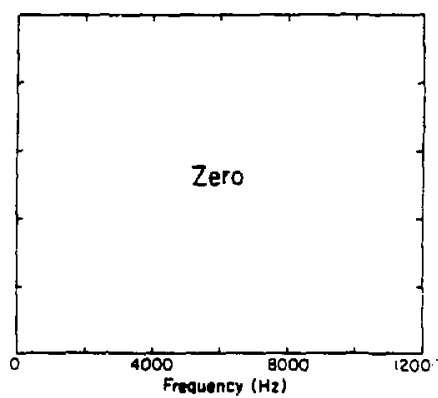

(c) Sine FT

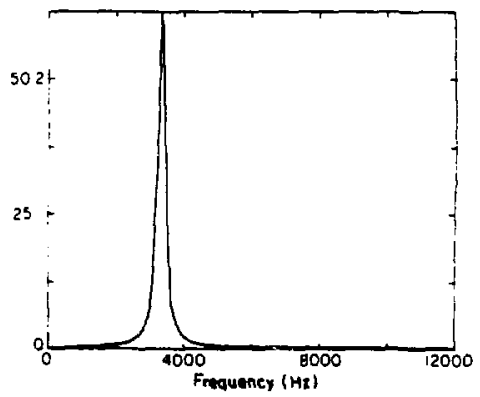

(e) Modulus FT

\section{Figure 15. Simulated Exponential Echo $(\phi=0)$ and its Fourier Transforms}




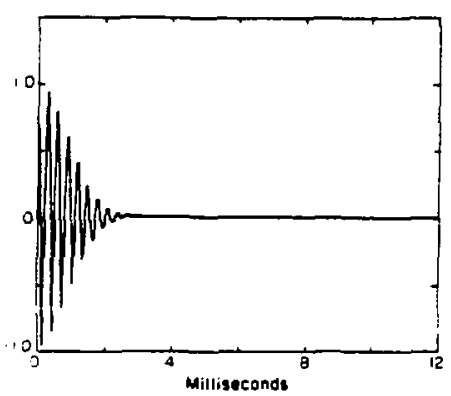

(o) Goussion FID, $\phi=0$

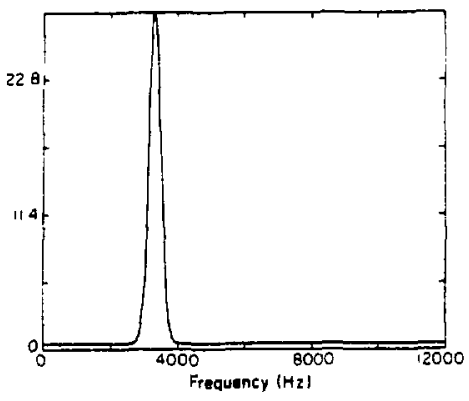

(b) Cosine FT

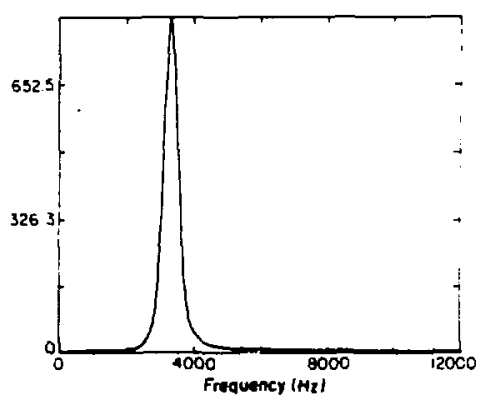

(d) Modulus Squored FT

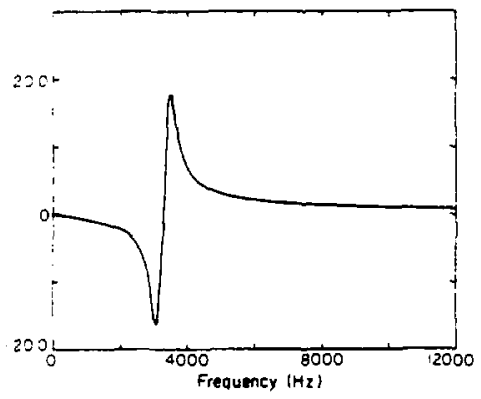

(c) Sine FT

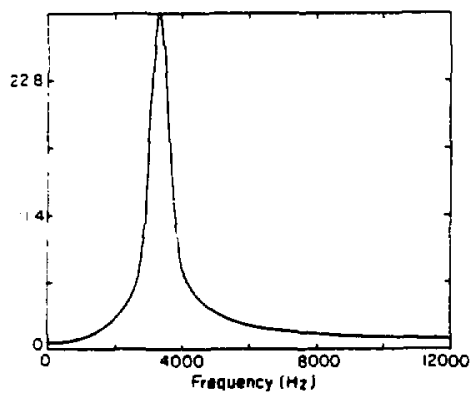

(e) Modulus FT

Figure 16. Simulated Gaussion FID $(\phi=0)$ and its Fourier Transforms 


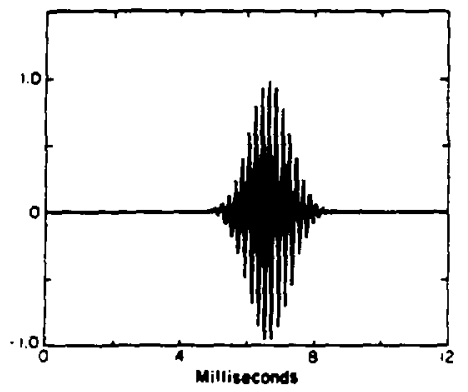

(a) Goussian Echo, $\phi=0$

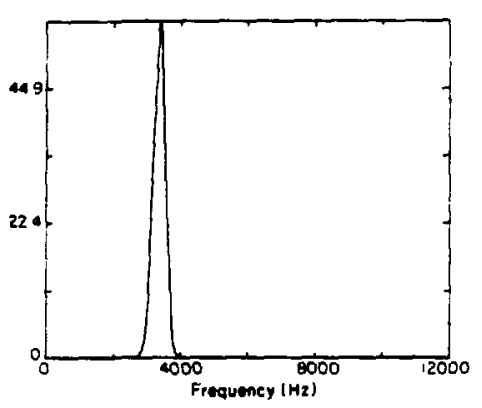

(b) Cosine FT

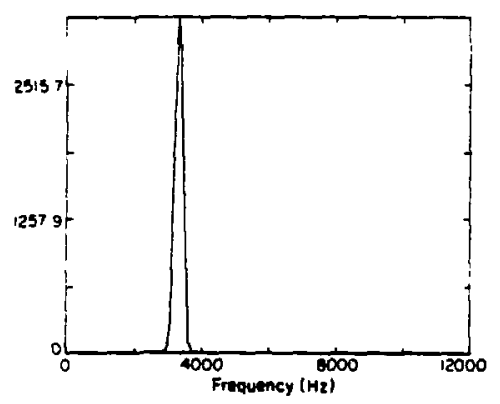

(d) Modulut Squond FT

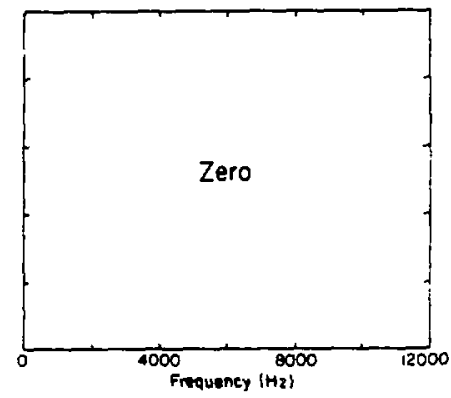

(c) Sine $F T$

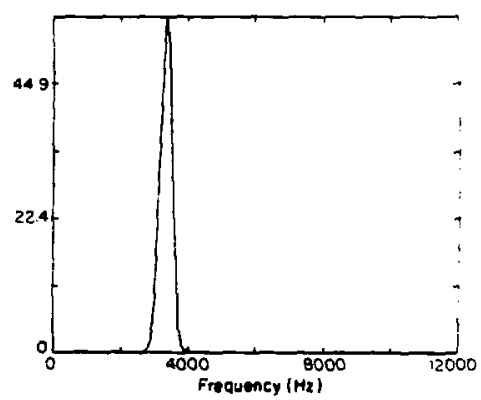

(e) Modulus FT

XOL $000-2722$

Figure 17. Simulated Goussion Echo $(\phi=0)$ and its Fourier Transforms 


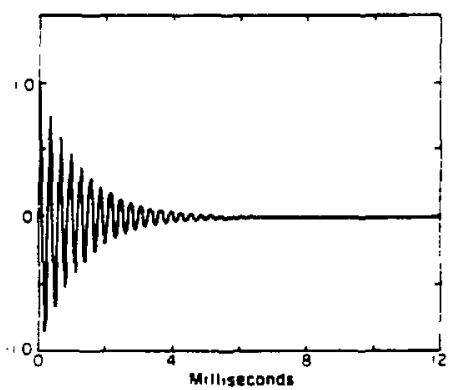

(o) Exponentıal FID, $\phi \propto 0$

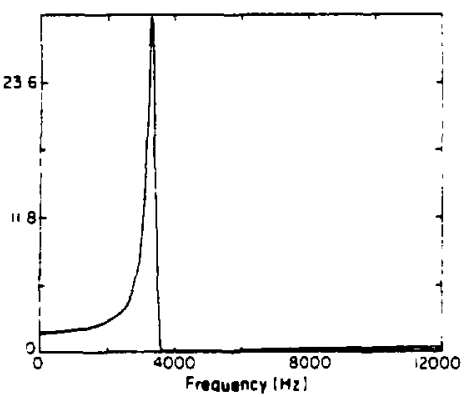

(b) Cosine FT

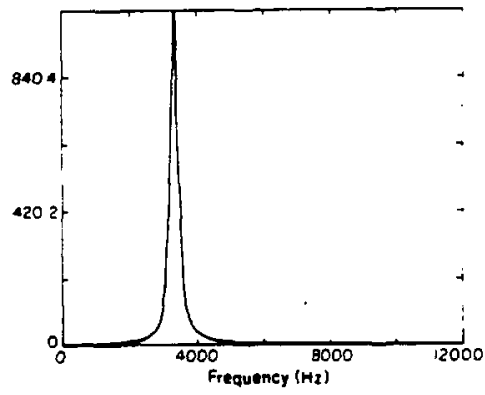

(d) Modulus Squoned FT

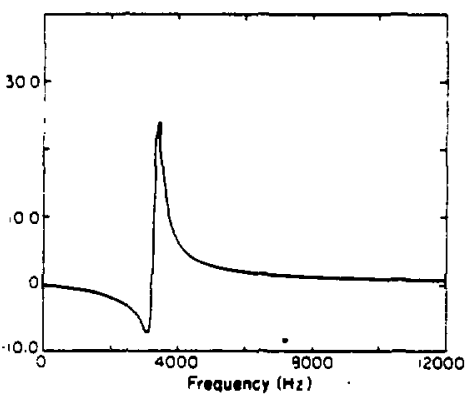

(c) Sine FT

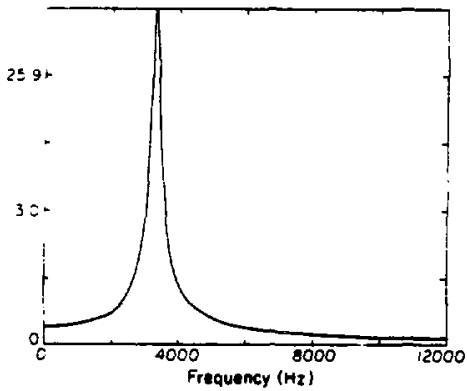

(e) Modulus FT

XeL $108-2712$ a

Figure 18. Simulated Exponential FID $(\phi \neq 0)$ and its Fourier Transforms 


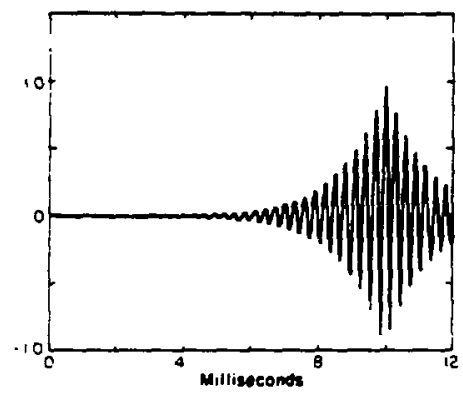

(o) Expanential Echo. $\phi * 0$

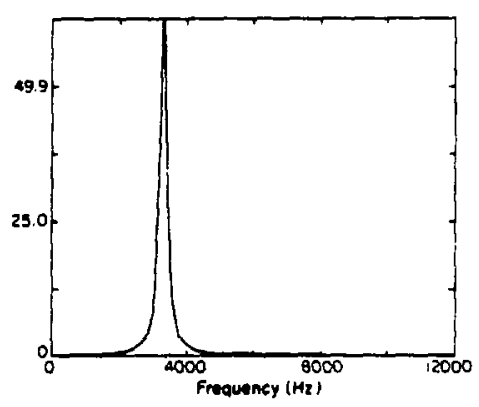

(b) Cosine FT

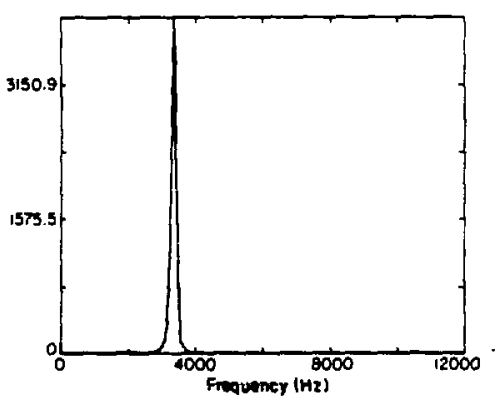

(d) Modulus Squond FT

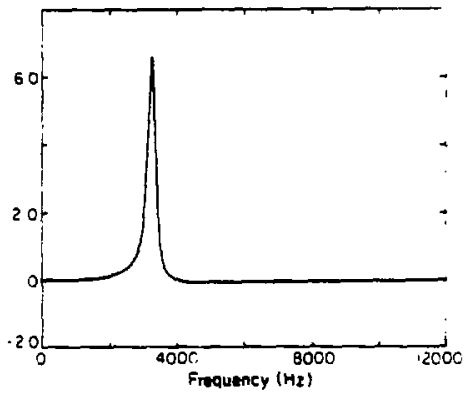

(c) Sine FT

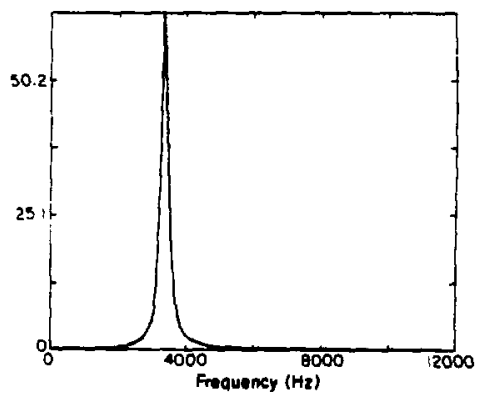

(e) Modulus FT

X6L $008-27138$

Figure 19. Simulated Exponential Echo $(\phi \neq 0)$ and its Fourier Transforms 


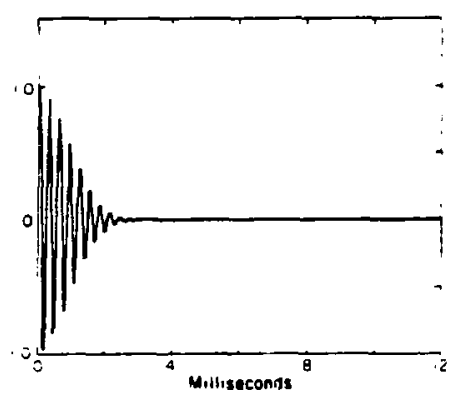

(o) Goussion FID. $\phi=0$

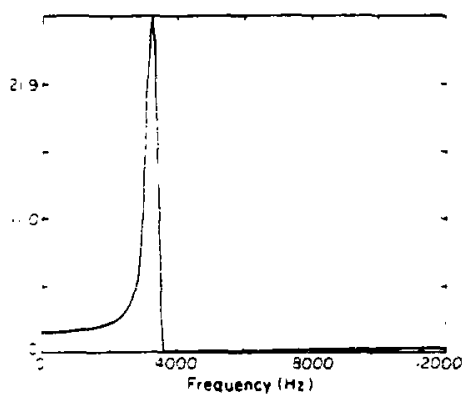

(b) Cosine FT

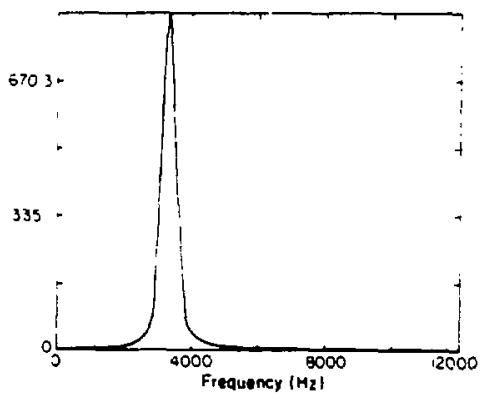

(d) Modulus Squared FT

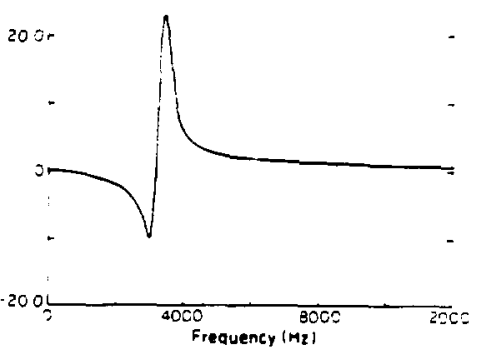

(c) Sine FT

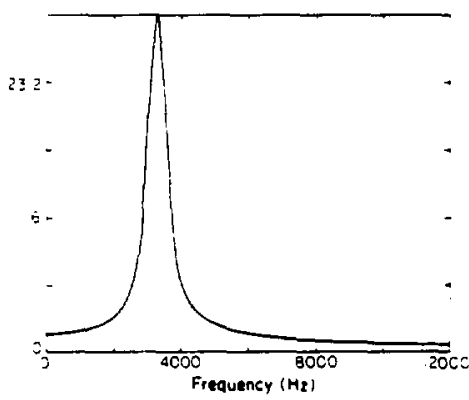

(e) Modulus FT

Figure 20. Simulated Gaussian FID $(\phi \neq 0)$ and its Fourier Transforms 


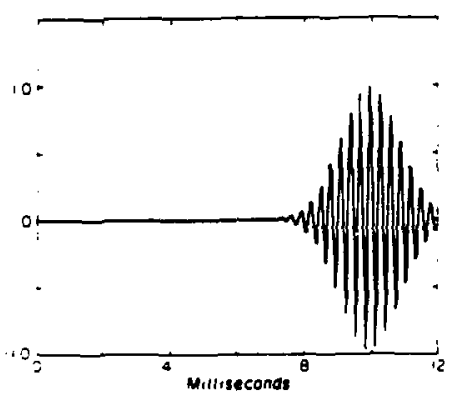

(o) Goussion Echo, $\phi \neq 0$

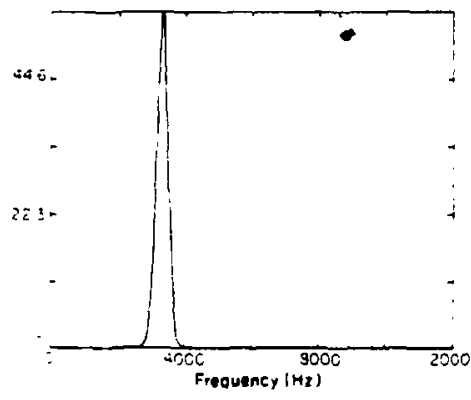

(b) Cosine FT

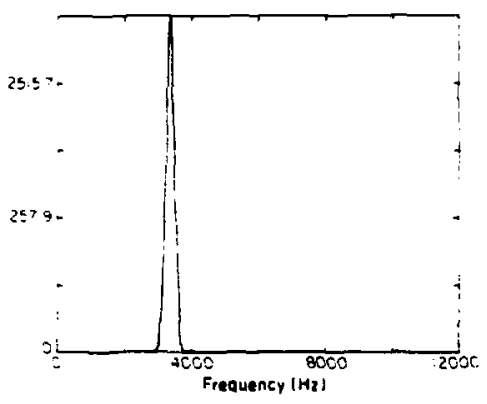

(d) Modulus Squared FT

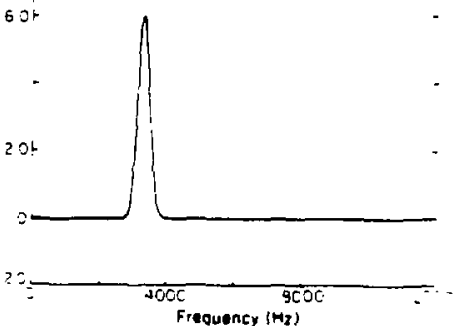

(c) Sine FT

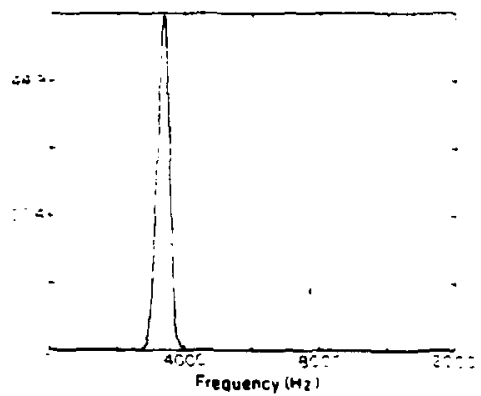

(e) Modulus FT

$\times 86$ 809. 2715

Figure 21. Simulated Gaussian Echo $(\phi \neq 0)$ and its Fourier Transforms 


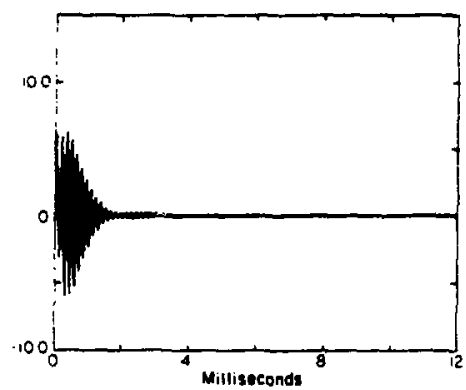

(a) Time Domain F10

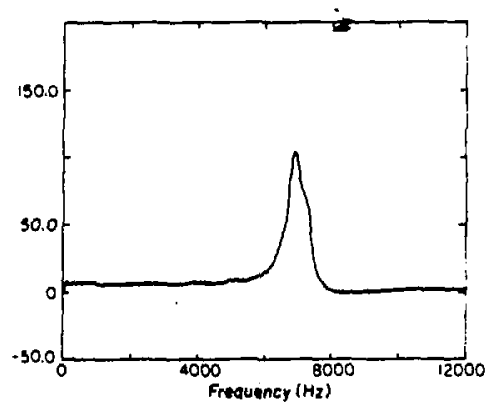

(b) Cosine FT

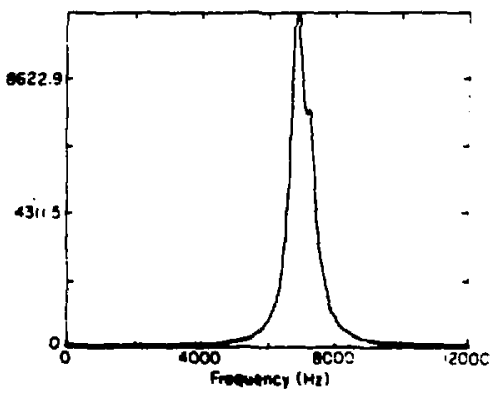

(d) Hodutw Spund FT

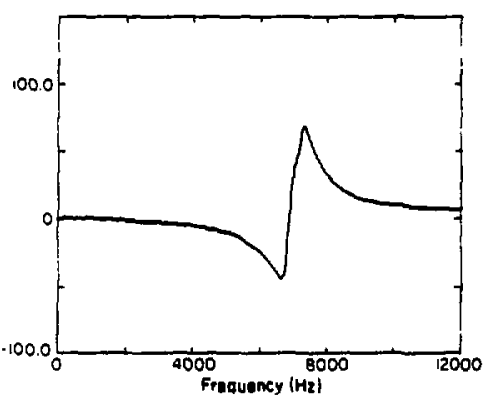

(c) Sine FT

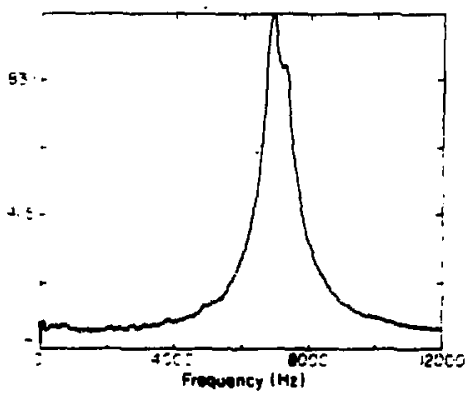

(a) Modulus FT

xh not-27ts

Figure 22. Hexamethylenetetramine, Room Temperature 


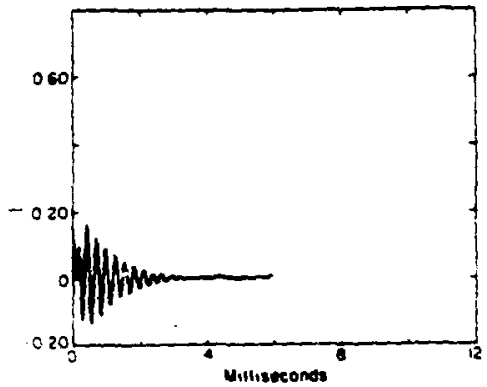

(o) Time Domain FiO

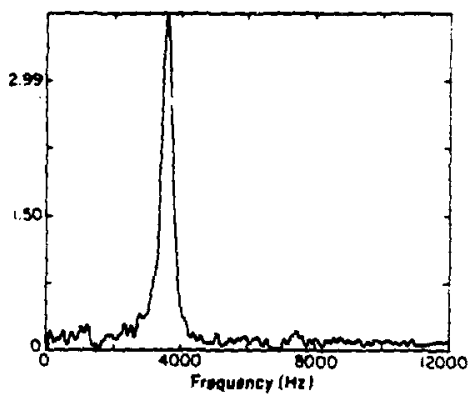

(b) Cosine FT

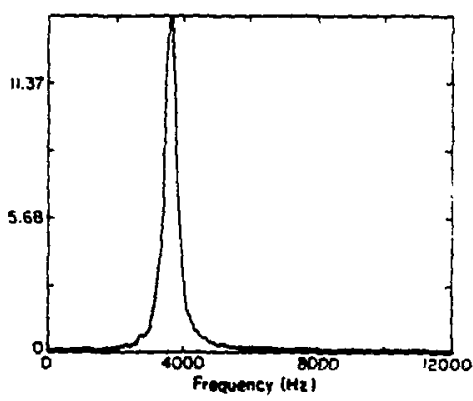

(d) Madulus Squored t

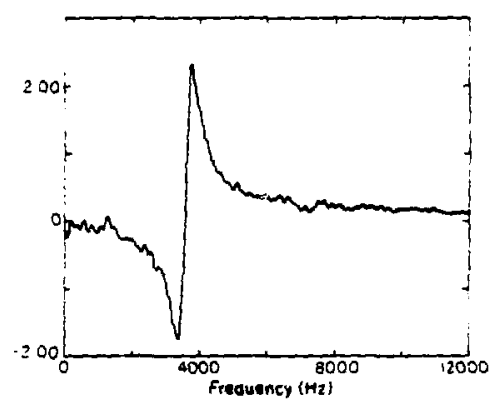

(c) Sine FT

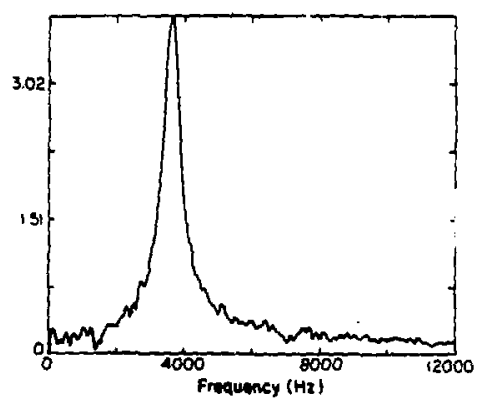

(e) Modulus FT

X8L 800-27174

Figure 23. Urea, $\nu_{+}$Line at $77^{\circ} \mathrm{K}$ 


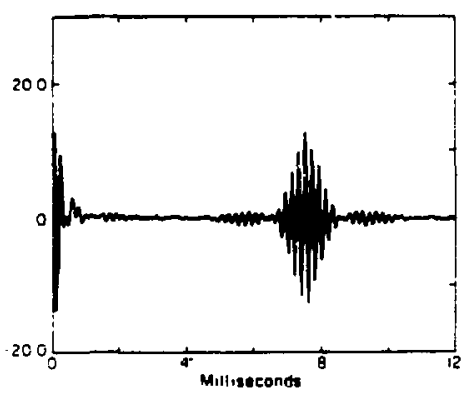

(o) Time Damain

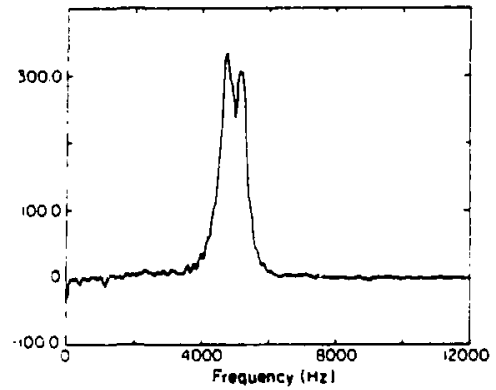

(b) Cosine FT

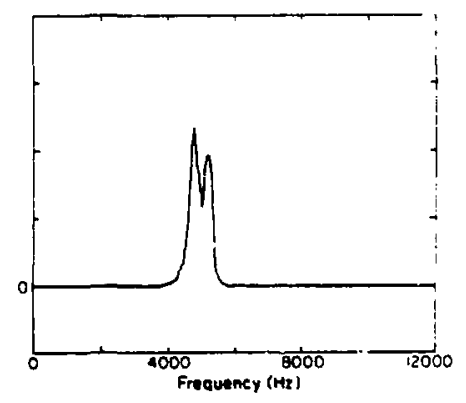

(d) Modulus Sque i, J FT

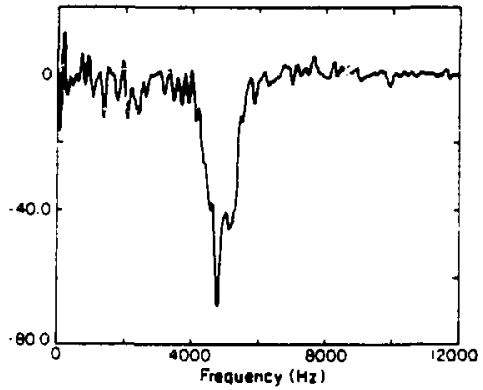

(c) Sine FT

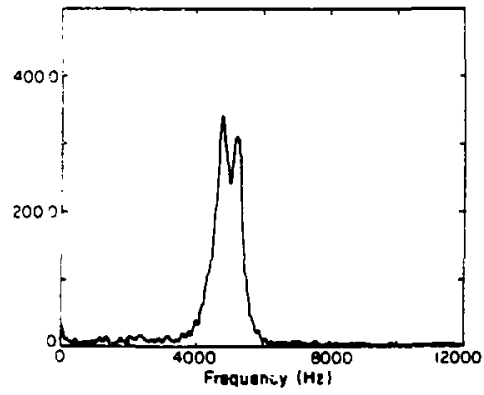

(e) Modulus FT

Figure $24.727 \mathrm{kHz}$ Doublet in Monoclinic TNT at $77^{\circ} \mathrm{K}$ 


\section{B. Axial Field of a Coil of Finite Height and Width}

If we define

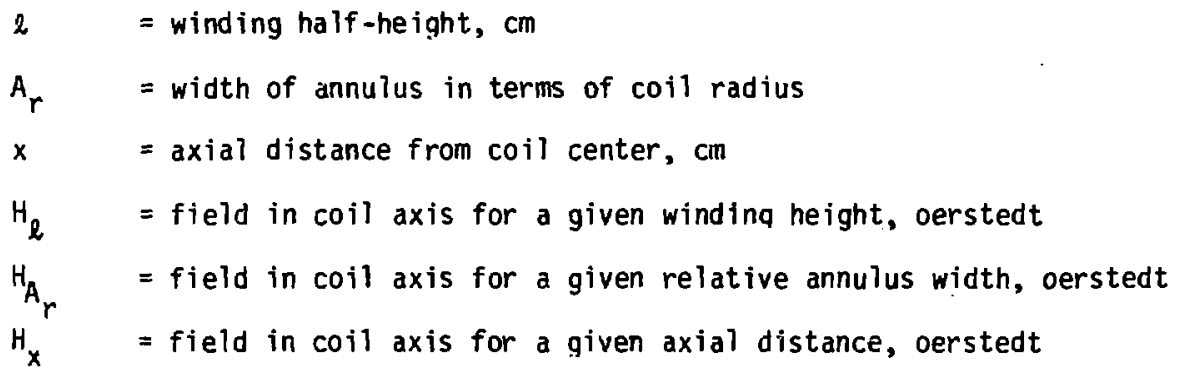

we have

$$
H_{0,0,0}=\frac{\pi}{s} \frac{n l}{r_{m}}
$$

where

$$
\begin{array}{ll}
n & =\text { turns in coil } \\
I & =\text { current in coil, amp } \\
r_{m} & =\text { median coil radius, cm } \\
r & =\text { coil radius, cm }
\end{array}
$$

Furthermore

$$
H_{r, \ell, x,}=\frac{\pi}{10} \frac{n I}{\ell}\left[\frac{x+\ell}{\sqrt{r^{2}+(x+\ell)^{2}}}-\frac{x-\ell}{\sqrt{r^{2}+(x-\ell)^{2}}}\right]
$$


and, if we make

$$
\begin{aligned}
& A=\frac{x+R}{r_{m}} \\
& B=\frac{x-R}{r_{m}}
\end{aligned}
$$

we have

$$
H_{Q, h}=\frac{\pi}{5} \quad \frac{n l}{A-B}\left[\frac{A}{\left(r^{2}+A_{m}^{2} r_{m}^{2}\right)^{1 / 2}}-\frac{B}{\left(r^{2}+B^{2} r_{m}^{2}\right)^{1 / 2}}\right]
$$

Now, we can describe the coil as a current sheet for which

$$
\frac{d H Q, X}{d r}=\frac{\pi}{5} \frac{1}{A-B}\left[\frac{A}{\left(r^{2}+A^{2} r_{m}^{2}\right)^{1 / 2}}-\frac{B}{\left(r^{2}+B^{2} r_{m}^{2}\right)^{1 / 2}}\right] \frac{d l}{d r}
$$

where

$$
\frac{\mathrm{d}}{\mathrm{dr}}=\text { linear current density in sheet, amp } \times \mathrm{cmp}^{-1}
$$

and we have by integration

$$
\frac{d I}{d r}=\frac{n I}{r_{m} A_{I}}
$$

we can make

$$
\begin{aligned}
& H_{Q, A_{r}, x}=\frac{\pi}{5} \frac{n I}{r_{m}^{2} A_{r}(A-B)}\left[A \int \frac{d r}{\left(r^{2}+A^{2} r_{m}^{2}\right)^{1 / 2}}-B \int \frac{d r}{\left(r^{2}+B^{2} r_{m}^{2}\right)^{1 / 2}}\right]_{r_{m}\left(1-A_{r / 2}\right)}^{r_{m}\left(1+A_{r / 2}\right)} \\
& =\frac{\pi}{5} \frac{n I}{r_{m}^{2} A_{r}(A-B)}\left[A R n \left(r+\sqrt{\left.r^{2}+A^{2} r_{m}^{2}\right)}-B Q n\left(r+\sqrt{\left.r^{2}+B^{2} r_{m}^{2}\right)}\right]_{r_{m}\left(1-A_{r / 2}\right)}^{r_{m}\left(1+A_{r / 2}\right)}\right.\right.
\end{aligned}
$$


C. EFFECT OF FINITE WINDING HEIGHT ON AXIAL FIELD OF TOROIDAL COIL

The axial field of a solenoidal coil is given by

$$
H_{Q}=\frac{2 \pi}{10} \frac{\mathrm{nl}}{l}\left[\frac{0.5 \ell+\mathrm{x}}{\sqrt{r^{2}+(0.5 l+x)^{2}}}+\frac{0.5 l-\mathrm{x}}{\sqrt{\mathrm{T}^{2}+(0.5 l-\mathrm{x})^{2}}}\right]
$$

where

$H_{\ell}=$ axial field for a given winding height, oerstedt

$n=$ number of turns in coil

$I=$ current in coil, amp

$\ell=$ winding height, $\mathrm{cm}$

$r$ = coil radius, $\mathrm{cm}$

$x=$ axial distance from coil center, $\mathrm{cm}$

If we make

$$
X=\frac{x}{r}
$$

where

$X=$ relative axial distance to coil center

and

$$
h=\frac{\ell}{2 r}
$$

where

$h=$ iength/diameter ratio of coil 
we have by t:ansposition

$$
H_{Q}=\frac{\pi}{10} \frac{n I}{r}\left[\frac{1+X / h}{\sqrt{1+(h+X)^{2}}}+\frac{1-X / h}{\sqrt{1+(h-X)^{2}}}\right]
$$

and since

$$
\lim _{l \rightarrow 0} H_{Q}=H_{0}=\frac{2 \pi}{10} \frac{1}{\left(I+X^{2}\right)^{3 / 2}}
$$

we can write

$$
\frac{H_{8}}{H_{0}}=\frac{1}{2}(1+X)^{3 / 2}\left[\frac{1+X / h}{\sqrt{1+(h+X)^{2}}}+\frac{1-X / h}{\sqrt{1+(h-X)^{2}}}\right]
$$

as the correction term sought. 
D. Effect of Finite Annular Width on Axial Field of Toroid.! Coil

$$
H=\frac{\pi}{5} \frac{l n}{\left.1+h^{2} / r_{m}^{2}\right)^{3 / 2} r_{m}}
$$

where

$$
\begin{aligned}
& H=\text { axial magnetic field, oerstedt } \\
& I=\text { current in coil, amp } \\
& r_{m}=\text { mean coil radius, cm } \\
& h=\text { axial distance from plane of coil, cm } \\
& n=\text { number of turns }
\end{aligned}
$$

To accomodate an annulus of finite width, we describe it as a current sheet for which

$$
n I=\int_{r_{m}\left(1-A_{t / 2}{ }_{r} d t\right.}^{r_{m}\left(1+A_{t / 2}\right)}
$$

where

$A_{r}=$ fractional annular width in terms of radius

$I_{r}=$ linear current density along radius, amp $\times \mathrm{cm}^{-1}$

giving

$$
\zeta=\frac{n I}{I_{m} A_{t}}
$$

Then

$$
d H=\frac{\pi}{5} \frac{I_{r}}{r\left(1+h^{2} / r^{2}\right)^{3 / 2}} d r
$$


and thus

$$
H^{\prime}=\frac{\pi}{5} \frac{n I}{r_{m} A_{t}} \int_{r_{m}\left(1-A_{r / 2}\right)}^{r_{m}\left(1+A_{r / 2}\right)} \frac{r^{2}}{\left(r^{2}+r^{2} \cdot 3 / 2\right.} d r
$$

where

$$
H^{\prime}=\text { axial field for finite width annulus. oerstedt }
$$

from which

$$
H^{\prime}=\frac{\pi}{5} \frac{n I}{r_{m}} \frac{1}{A_{t}}\left[\ln \left(r+\sqrt{r^{2}+h^{2}}\right)-\frac{r}{\sqrt{r^{2}+h^{2}}}\right]_{r_{m}\left(1-A_{r / 2}\right)}^{r_{m}\left(i+A_{r / 2}\right)}
$$

and thus

$$
\begin{aligned}
& H^{\prime}=\frac{\pi}{5} \frac{n I}{r_{m} A_{r}}\left[\ln \frac{1+A_{r / 2}+\sqrt{\left(1+A_{r / 2}\right)^{2}+h^{2} / r_{m}^{2}}}{1-A_{r / 2}} \frac{\sqrt{\left(1-A_{r / 2}\right)^{2}+h^{2} / r_{m}^{2}}}{\sqrt{2}}+\right. \\
& \left.\frac{\left(1-A_{r / 2}\right)}{\sqrt{\left(1-A_{r / 2}\right)^{2}+h^{2} / r_{m}^{2}}}-\frac{\left(1+A_{r / 2}\right)}{\sqrt{\left(1+A_{r / 2}\right)^{2}+h^{2} / r_{m}^{2}}}\right]
\end{aligned}
$$

giving

$$
\begin{aligned}
\frac{H^{\prime}}{H}= & \frac{\left(1+h^{2} / r_{m}^{2}\right)^{3 / 2}}{A_{r}}\left[\ln \frac{1+A_{r / 2}-\sqrt{\left(1+A_{r / 2}\right)^{2}+h^{2} / r_{m}^{2}}}{1-A_{r / 2}-\sqrt{\left(1-A_{r / 2}\right)^{2}+h^{2} / r_{m}^{2}}}+\right. \\
& \left.\frac{\left(1-A_{t / 2}\right)}{\sqrt{\left(1-A_{r / 2}\right)^{2}+h^{2} / r_{m}^{2}}}-\frac{\left(1+A_{r / 2}\right)}{\sqrt{\left(1+A_{r / 2}\right)^{2}+h^{2} / r_{m}^{2}}}\right]
\end{aligned}
$$




\section{E. Opt imun HQR Coil for Constant Maximum Field}

\section{Given}

$F_{\text {MAX }}=$ maximum allowable electric field between coil terminals, $V \mathrm{~cm}^{-1}$ we have

$$
V_{\text {MAX }}=F_{\text {MAX }} r A_{r} F_{a}
$$

where

$$
\begin{aligned}
& V_{\text {MaX }}=\text { maximum voltage across coil terminals, } v \\
& r=\text { coil radius, } c m \\
& A_{r}=\text { fractional width of annulus in terms of radius } \\
& F_{a}=\text { fraction of annulus occupied by air gaps }
\end{aligned}
$$

We al so have

$$
V_{\operatorname{MAX}}=4_{\operatorname{MAX}} Z=I_{\operatorname{MAX}} \sqrt{\frac{L}{C}}
$$

where

$$
\begin{aligned}
& I_{\text {MAX }}=\text { maximum current, in coil, } A \\
& Z=\text { impedance, } \Omega \\
& L=\text { inductance, } H \\
& C=\text { capacitance, } F
\end{aligned}
$$

where, for a sample located axially one radius below the coil plane

$$
I_{\text {MAX }}=\frac{10 \sqrt{2}}{\pi} \frac{\mathrm{r}}{\mathrm{n}} \mathrm{H}_{\mathrm{MAX}}
$$

$$
n=\text { number of turns in coil }
$$

$H_{\text {MAX }}=$ maximum magnetic field at sample, oe in which, for a quasi-rectangular pulse

$$
H_{\text {MAX }}=\frac{\pi}{2} \frac{1}{\gamma t_{p}}
$$




\section{E-2}

where

$$
\begin{aligned}
\gamma & =\text { grromagnetic ratio, } \operatorname{rad}^{-1} \mathrm{sec}^{-1} \\
t_{p} & =\text { pulse length, } \mathrm{sec}
\end{aligned}
$$

Then

$$
\mathrm{J}_{\mathrm{MAX}}=\frac{10}{\sqrt{2}} \frac{\mathrm{r}}{n} \quad \frac{1}{\gamma \mathrm{t}_{\mathrm{p}}}
$$

and

$$
V_{\text {MAX }}=\frac{10}{\sqrt{2}} \frac{r L^{1 / 2}}{h C^{1 / 2}} \frac{1}{\gamma t_{p}}
$$

Now, the resonance condition

$$
f=\frac{1}{2 \pi \sqrt{L C}}
$$

where

$$
f=\text { frequency, } \mathrm{Hz}
$$

reduces to

$$
\sqrt{\frac{L}{C}}=\frac{1}{2 \pi C}
$$

from which

$$
\mathrm{V}_{\mathrm{MAX}}=\frac{5}{\sqrt{2} \pi} \frac{\mathrm{r}}{\mathrm{nfC}} \frac{1}{\gamma \mathrm{t}_{\mathrm{p}}}
$$


Now, we can also wite

$$
C=\frac{5 \times 10^{8}}{c^{2}} \frac{h_{D} K_{m}}{F_{c} S_{c}}
$$

where

$$
\begin{aligned}
& h_{D}=\text { height of coil ribbon conductor, } \mathrm{cm} \\
& K_{m}=\text { mean dielectric constant of gap } \\
& F_{C}=\text { fraction of total capacitance inside coil } \\
& S_{c}=\text { gap width in terms of radius } \\
& c=\text { velocity of light, } \mathrm{cm} \mathrm{sec}-1
\end{aligned}
$$

and also

$$
S_{c}=\frac{A_{r} F_{a}}{n}
$$

from which

$$
c=\frac{5 \times 10^{8}}{c^{2}} \frac{n h_{D} K_{m}}{F_{c} A_{r} F_{a}}
$$

giving

$$
V_{\text {MAX }}=\frac{10^{-8} c^{2}}{\sqrt{2} \pi} \frac{r A_{t} F_{a} F_{c}}{n^{2} h_{D} K_{m} f} \frac{1}{\gamma t_{p}}
$$

Combining this with Equation 1 gives

$$
n=\frac{10^{-4} c}{2^{1 / 4} \pi^{1 / 2}} \frac{F_{c}^{1 / 2}}{\left.F_{M A X}^{1}\right|_{D} ^{2} h_{D}^{1 / 2} K_{m}^{1 / 2} f^{1 / 2}} \frac{1}{\gamma^{1 / 2} t_{p}^{1 / 2}}
$$


from which by substitution

$$
\begin{aligned}
& S_{c}=\frac{2^{1 / 4} \pi^{1 / 2} 10^{4}}{c} \frac{A_{f} F_{2} F_{M A X}^{1 / 2} h_{D}^{1 / 2} K_{m}^{1 / 2} f^{1 / 2}}{F_{c}^{1 / 2}} \gamma^{1 / 2} t_{p}^{1 / 2} \\
& C=\frac{5 \times 10^{4}}{2^{1 / 4} \pi^{1 / 2} c} \frac{h_{D}^{1 / 2} K_{m}^{1 / 2}}{F_{c}^{1 / 2} A_{f} F_{a} F_{M A X}^{1 / 2} f^{1 / 2}} \frac{1}{\gamma^{1 / 2} t_{p}^{1 / 2}}
\end{aligned}
$$

Now, since

$$
L=\frac{K_{m^{2}}^{2}}{F_{L}}
$$

where

$$
\begin{aligned}
& K=\text { coil factor, } \mathrm{H} \mathrm{cm}^{-1} \\
& F_{L}=\text { fraction of inductance in coil }
\end{aligned}
$$

we have

$$
\mathrm{L}=\frac{10^{-8} \mathrm{c}^{2}}{\sqrt{2 \pi}} \frac{\mathrm{rKF}_{\mathrm{c}}}{\mathrm{h}_{\mathrm{D}} \mathrm{F}_{\mathrm{MAX}} \mathrm{K}_{\mathrm{m}} \mathrm{FF}_{\mathrm{L}}} \frac{1}{\gamma \mathrm{t}_{\mathrm{p}}}
$$

On the other hand, from Equation 7, we have

$$
L=\frac{1}{4 \pi^{2}} \quad \frac{1}{f^{2} C}
$$

and therefore

$$
L=\frac{c}{2^{3 / 4} 10^{5} \pi^{3 / 2}} \frac{F_{c}^{1 / 2} A_{r} F_{a} F_{M A X}^{1 / 2}}{h_{D}^{1 / 2} K_{m}^{1 / 2} f^{3 / 2}} \gamma^{1 / 2} t_{p}^{1 / 2}
$$


from which

$$
h_{D}=10^{-6} c^{2} \sqrt{2 \pi} \frac{r^{2} F_{c} \mathrm{XX}^{2}}{A_{r}^{2} F_{a}^{2} K_{m} F_{M A X}^{3} F_{L}^{2}} \frac{1}{\gamma^{3} t_{p}^{3}}
$$

He now substitute again and have

$$
\begin{aligned}
& n=\frac{1}{10 \sqrt{2} \pi} \frac{A_{\mathrm{r}} \mathrm{F}_{\mathrm{a}} \mathrm{F}_{\mathrm{MAx}} \mathrm{F}_{\mathrm{L}}}{\mathrm{rKf}} \gamma \mathrm{t}_{\mathrm{p}} \\
& S_{c}=10 \sqrt{2} \pi \frac{\tau \mathrm{Kf}}{F_{\text {MAX }} F_{L}} \quad \frac{1}{\gamma t_{p}} \\
& C=50 \frac{r K}{A_{\mathrm{I}}^{2} \mathrm{~F}_{\mathrm{a}}^{2} \mathrm{~F}_{\mathrm{MAX}}^{2} \mathrm{~F}_{\mathrm{L}}} \frac{1}{\gamma^{2} \mathrm{t}_{\mathrm{P}}^{2}} \\
& L=\frac{1}{200 \pi^{2}} \frac{A_{T}^{2} F_{a}^{2} F_{M A X}^{2} F_{L}}{\mathrm{Krf}^{2}} r_{p}^{2} t_{p}^{2} \\
& Z=\frac{10^{-2}}{\pi} \frac{A_{\mathrm{r}}^{2} \mathrm{~F}_{\mathrm{a}}^{2} \mathrm{~F}_{\mathrm{MAX}}^{2}}{\mathrm{I} \mathrm{Kf}} \gamma_{\mathrm{p}}^{2} \mathrm{q}_{\mathrm{p}}^{2}
\end{aligned}
$$

giving

$$
I_{\text {MAX }}=100 \pi \frac{r^{2} K f}{A_{r} F_{a} F_{M A X} F_{L}} \frac{1}{\gamma^{2} t_{p}^{2}}
$$

There

$$
\left(\mathrm{P}_{\mathrm{MAX}}\right)_{\mathrm{CIRC}}=50 \pi \frac{\mathrm{r}^{3} \mathrm{Kf}}{\mathrm{F}_{\mathrm{L}}} \frac{1}{r^{2} \mathrm{t}_{\mathrm{p}}^{2}}
$$

where

$\left(P_{\text {MAX }}\right)_{C I R C}=$ maximum circulating power, $W$

He now turn to the dissipative losses in the coil, where

$$
R=\pi \frac{r n \rho}{h_{D} \sigma F_{R}}
$$




\section{E-6}

where

$$
\begin{aligned}
R & =\text { resistance, } \Omega \\
\rho & =\text { conductor resistivity, } \Omega \mathrm{cm}^{-1} \\
\sigma & =\text { skin depth, } \mathrm{cm} \\
F_{R} & =\text { fraction of total resistance in coil }
\end{aligned}
$$

and, in which

$$
\sigma=\frac{10^{4} \sqrt{10}}{2 \pi} \frac{\rho^{1 / 2}}{f^{1 / 2}}
$$

Then

$$
R=\frac{\sqrt{10}}{c^{2}} \frac{A_{\mathrm{T}}^{3} F_{\mathrm{a}}^{3} F_{\mathrm{MAX}}^{4} F_{\mathrm{L}}^{3} K_{\mathrm{m}} \rho^{1 / 2}}{r^{2} \mathrm{~K}^{3} F_{\mathrm{c}} F_{\mathrm{R}} f^{3 / 2}} \gamma_{\mathrm{p}}^{4} \mathrm{t}^{4}
$$

and since

$$
\mathrm{Q}=\frac{2 \pi \mathrm{fL}}{\mathrm{R}}
$$

where

$$
Q=Q-f a c t o r
$$

we have

$$
Q=\frac{10^{-3} 10 c^{2}}{\pi} \frac{\mathrm{rK}^{2} F_{c} F_{R} f^{1 / 2}}{A_{r} F_{a} F_{M A X}^{2} F_{L}^{2} K_{m} \rho^{1 / 2}} \frac{1}{\gamma^{2} t_{p}^{2}}
$$

On the other hand, we have

$$
\left(P_{\text {MAX }}\right)_{\text {DIS }}=\frac{1}{2} I_{\text {MAX }}^{2} R
$$


where

$\left(P_{\text {max }}\right\rangle_{D I S}=$ maximum power dissipation rate, $W$, from which

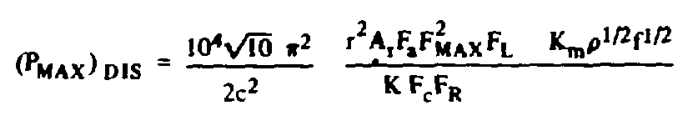

Furthermore

$$
E_{\text {MAX }}=\frac{1}{2} \quad I_{\text {MAX }}^{2} \quad L
$$

where

$$
\begin{gathered}
E_{\text {MAX }}=\text { maximum energy storage in field, } J \text {, from which } \\
E_{M A X}=25 \frac{\mathrm{s}^{3} \mathrm{~K}}{\mathrm{~F}_{\mathrm{L}}} \frac{1}{\gamma^{2} \mathrm{t}_{\mathrm{p}}^{2}}
\end{gathered}
$$

Finally, for a quasirectangular pulse

$$
\left(P_{\text {MAX }}\right)_{\text {LOAD }}=\frac{2}{t_{p}} \quad E_{\text {MAX }}
$$

where

$\left(P_{\text {MAX }}\right)_{L O A D}=$ maximum power input into coil, $W$, from which

$$
\left(\mathbf{P}_{\text {MAX }}\right)_{\text {LOAD }}=50 \frac{\mathrm{r}^{3} \mathrm{~K}}{F_{L}} \frac{1}{\gamma^{2} t_{P}^{3}}
$$

As an example, let us take

$$
\begin{aligned}
& A_{r}=0.40 \\
& F_{a}=F_{L}=F_{c}=F_{R}=0.90 \\
& F_{\operatorname{mX}}=6000 \vee \mathrm{cm}^{-1}
\end{aligned}
$$




$$
\begin{aligned}
K_{m} & =2.26 \\
K & =3.5 \times 10^{-8} \mathrm{H} \mathrm{cm}^{-1} \\
\rho & =1.62 \times 10^{-6} \Omega \mathrm{cm}^{-1}(\mathrm{Ag}) \\
\gamma & =1640 \mathrm{rad} \mathrm{oe}-1 \mathrm{sec}^{-1} \\
t_{p} & =250 \mu \mathrm{sec} \\
f & =3.41 \mathrm{MHz}
\end{aligned}
$$

\begin{tabular}{|c|c|c|c|c|c|}
\hline$r$ & $10 \mathrm{~cm}$ & 15 & 20 & 25 & 30 \\
\hline$n$ & 15.03 & 10.02 & 7.515 & 6.012 & 5.010 \\
\hline$S_{c}$ & $2.39 \times 10^{-2}$ & $3.595 \times 10^{-2}$ & $4.790 \times 10^{-2}$ & $5.988 \times 10^{-2}$ & $7.185 \times 10^{-}$ \\
\hline$h_{D}$ & $4.251 \mathrm{~mm}$ & 9.565 & 17.00 & 26.57 & 38.26 \\
\hline$\sigma$ & $34.69 \mu \rightarrow$ & & & & \\
\hline$H_{\text {MAX }}$ & 3.831 oe $\rightarrow$ & & & & \\
\hline$L$ & $87.96 \mu \mathrm{H}$ & 58.57 & 43.93 & 35.14 & 29.29 \\
\hline C & $24.79 \mathrm{pF}$ & 37.19 & 49.58 & 61.98 & 74.37 \\
\hline$R$ & $0.5764 \Omega$ & 0.2562 & 0.1441 & $0.092 ? 2$ & 0.06404 \\
\hline$Q$ & 3266 & 4899 & 6532 & 8165 & 9798 \\
\hline$V_{\text {MAX }}$ & $21.60 \mathrm{kV}$ & 32.40 & 43.20 & 54.00 & 64.80 \\
\hline$I_{\text {MAX }}$ & 11.47 & 26.42 & 46.96 & 73.38 & 105.7 \\
\hline$E_{\text {MAX }}$ & $5.784 \mathrm{~mJ}$ & 19.52 & 46.27 & 90.38 & 156.2 \\
\hline d & $2.053 \mathrm{~mm}$ & 5.389 & 9.580 & 1497 & 21.56 \\
\hline$z$ & $1882 \Omega$ & 1255 & 941.0 & 752.8 & 672.3 \\
\hline$\left(P_{\text {MOX }}\right)_{C I R C}$ & $123.9 \mathrm{KW}$ & 418.2 & 991.2 & 1936 & 3345 \\
\hline$\left(P_{\text {MXX }}\right)_{\text {DIS }}$ & $37.92 \mathrm{~W}$ & 85.32 & 151.7 & 237.0 & 341.3 \\
\hline$\left(P_{M A X}\right)_{L O A D}$ & $46.27 \mathrm{~W}$ & 156.2 & 370.2 & 723.0 & 1249 \\
\hline
\end{tabular}

We then get 


\section{F. Noil Coptimization}

\section{Given}

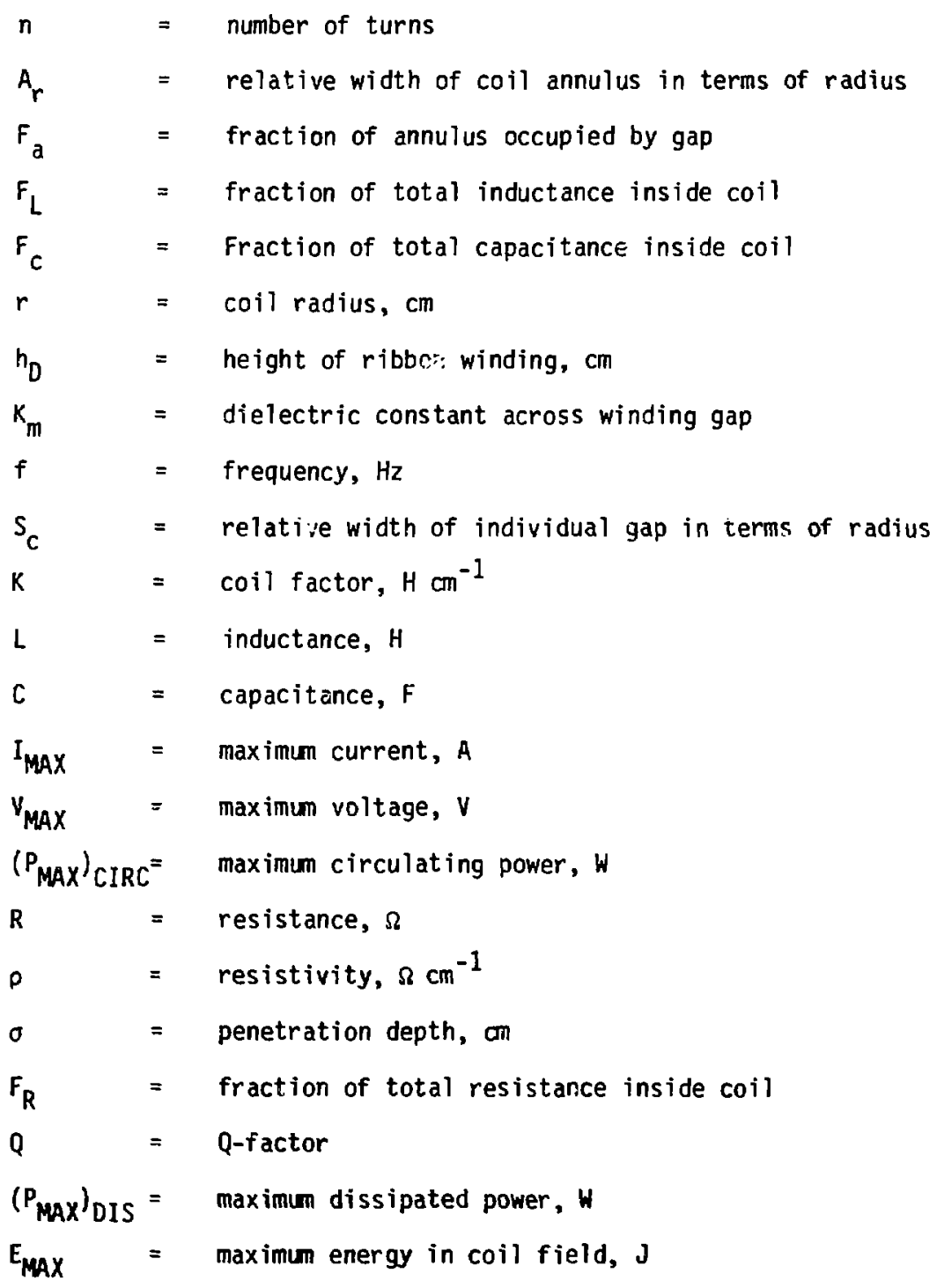


thax $_{\text {MAX }}=$ maxiumum magnetic field on coil axis one radius belon coij plane, oe

d $\quad=$ gap width, $\mathrm{cm}$

$Y=$ gyromagnetic ratio, $\mathrm{rad} \mathrm{oe}^{-1} \sec ^{-1}$

$t_{p} \quad=$ pulse length, sec

$c=$ velocity of light, $\mathrm{cm} \mathrm{sec}-1$

$F_{\text {MAX }}=$ maximum field between coi 1

$z=$ impedance, $\Omega$

we have, for an axial sunple one radius away from the coil plane

$$
n=\frac{10^{-3} c^{2 / 3}}{2^{1 / 3} \pi^{2 / 3}} \frac{A_{r}^{1 / 3} F_{a}^{1 / 3} F_{L}^{1 / 3} F_{c}^{1 / 3}}{r^{1 / 3} h_{D}^{1 / 3} K_{m}^{1 / 3} f^{2 / 3} K^{1 / 3}}
$$

$S_{c}=\frac{10^{3} 2^{1 / 3} \pi^{2 / 3}}{c^{2 / 3}} \frac{\mathrm{r}^{1 / 3} h_{D}^{1 / 3} A_{\mathrm{r}}^{2 / 3} \mathrm{~F}_{\mathrm{a}}^{2 / 3} \mathrm{~K}_{\mathrm{m}}^{1 / 3} \mathrm{~K}^{1 / 3} \mathrm{f}^{2 / 3}}{\mathrm{~F}_{\mathrm{L}}^{1 / 3} \mathrm{~F}_{\mathrm{c}}^{1 / 3}}$

$L=\frac{10^{-6} c^{4 / 3}}{2^{2 / 3} \pi^{4 / 3}} \frac{r^{1 / 3} A_{r}^{2 / 3} F_{a}^{2 / 3} F_{c}^{2 / 3} K^{1 / 3}}{h_{D}^{2 / 3} K_{m}^{2 / 3} F_{L}^{1 / 3} f^{4 / 3}}$

$$
\mathrm{C}=\frac{10^{6}}{2^{4 / 3} \pi^{2 / 3} \mathrm{c}^{4 / 3}} \frac{\mathrm{F}_{\mathrm{L}}^{1 / 3} \mathrm{~h}_{\mathrm{D}}^{2 / 3} \mathrm{~K}_{\mathrm{m}}^{2 / 3}}{\mathrm{r}_{\mathrm{r}}^{1 / 3} \mathrm{~F}_{\mathrm{c}}^{2 / 3} \mathrm{~A}_{\mathrm{r}}^{2 / 3} \mathrm{~F}_{\mathrm{a}}^{2 / 3} \mathrm{~K}^{1 / 3} \mathrm{f}^{2 / 3}}
$$




$$
\sigma=\frac{10^{4} \sqrt{10}}{2 \pi} \frac{\rho^{1 / 2}}{f^{1 / 2}}
$$

$$
R=10^{-8} 2^{2 / 3} \sqrt{10} \pi^{4 / 3} c^{2 / 3} \frac{r^{2 / 3} A_{r}^{1 / 3} F_{a}^{1 / 3} F_{L}^{1 / 3} F_{c}^{1 / 3} \rho^{1 / 2}}{h_{D}^{1 / 3} f^{1 / 6} F_{R} K_{m}^{1 / 3} K^{1 / 3}}
$$

$$
Q=\frac{10 \sqrt{10} c^{2 / 3}}{\pi^{5 / 3} 2^{1 / 3}} \frac{h_{D}^{2 / 3} A_{r}^{1 / 3} F_{a}^{1 / 3} F_{c}^{1 / 3} F_{R} k^{2 / 3}}{r^{1 / 3} F_{L}^{2 / 3} \rho^{1 / 2} K_{m}^{1 / 3} f^{1 / 6}}
$$

$$
H_{\text {MAX }}=\pi \frac{1}{\gamma t_{p}} \text { (quasirectangular pulse) }
$$

$$
\begin{aligned}
& I_{\mathrm{MAX}}=\frac{10^{4} \pi^{2 / 3}}{2^{1 / 6} c^{2 / 3}} \frac{\mathrm{r}^{4 / 3} \mathrm{~h}_{\mathrm{D}}^{1 / 3} \mathrm{~K}_{\mathrm{m}}^{1 / 3} K^{1 / 3} \mathrm{f}^{2 / 3}}{\mathrm{~A}_{\mathrm{r}}^{1 / 3} \mathrm{~F}_{\mathrm{a}}^{1 / 3} \mathrm{~F}_{\mathrm{L}}^{1 / 3} \mathrm{~F}_{\mathrm{c}}^{1 / 3} \gamma \mathrm{t}_{\mathrm{p}}} \\
& V_{k: A X}=2^{1 / 6} 10^{-2} c^{2 / 3} \pi^{1 / 3} \frac{5^{5 / 3} A_{s}^{1 / 3} F_{a}^{1 / 3} F_{r}^{1 / 3} K^{2 / 3} f^{1 / 3}}{h_{D}^{1 / 3} K_{m}^{1 / 3} F_{L}^{2 / 3} r_{p}}
\end{aligned}
$$

$$
\left(\mathrm{P}_{\text {MAX }}\right)_{\text {CIRC }}=50 \pi \frac{\mathrm{r}^{3} \mathrm{~K} \mathbf{f}}{\mathrm{F}_{\mathrm{L}} \boldsymbol{\gamma}^{2} \mathrm{t}_{\mathrm{p}}^{2}}
$$




$$
\begin{aligned}
& \text { F }-4
\end{aligned}
$$

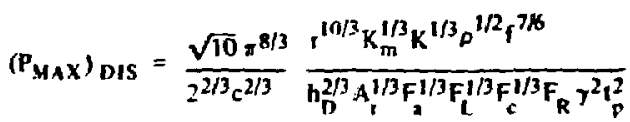

$$
\begin{aligned}
& F_{\text {MAX }}=2^{7 / 6} 10^{-2} c^{2 / 3} \pi^{1 / 3} \frac{r^{2 / 3} F_{c}^{1 / 3} K^{2 / 3} f^{1 / 3}}{h_{b}^{1 / 3} A_{t}^{2 / 3} F_{3}^{2 / 3} K_{m}^{1 / 3} F_{L}^{2 / 3} \gamma_{p}} \\
& E_{\text {MAX }}=25 \frac{\mathrm{r}^{3} \mathrm{~K}}{\mathrm{~F}_{\mathrm{L}} \gamma^{2} \mathrm{~T}_{\mathrm{P}}^{2}} \\
& \left(P_{M A X}\right)_{L O A D}=100 \pi \frac{r^{3} \mathrm{~K}}{F_{L} \gamma^{2} t_{p}^{3}} \\
& d=\frac{10^{3} \pi^{2 / 3} 2^{1 / 3}}{\mathrm{C}^{2 / 3}} \frac{{ }^{4 / 3} h_{D}^{1 / 3} A_{t}^{2 / 3} F_{a}^{2 / 3} K_{m}^{1 / 3} K^{1 / 3} f^{2 / 3}}{F_{L}^{1 / 3} F_{c}^{1 / 3}} \\
& \mathrm{z}=\frac{10^{-6} \mathrm{c}^{4 / 3} 2^{1 / 3}}{\pi^{1 / 3}} \frac{\mathrm{r}^{1 / 3} \mathrm{~A}_{\mathrm{f}}^{2 / 3} \mathrm{~F}_{\mathrm{a}}^{2 / 3} \mathrm{~F}_{\mathrm{c}}^{2 / 3} \mathrm{~K}^{1 / 3}}{\mathrm{~h}_{\mathrm{D}}^{2 / 3} \mathrm{~K}_{\mathrm{m}}^{2 / 3} \mathrm{~F}_{\mathrm{L}}^{1 / 3} \mathrm{f}^{1 / 3}}
\end{aligned}
$$


$\frac{V_{\text {MAX }}}{n}=10 \sqrt{2}: \frac{\mathrm{r}^{2} \mathrm{~K}}{\mathrm{~F}_{\mathrm{L}} \mathrm{r}_{\mathrm{P}}}$

As an exanple, for

$\begin{array}{ll}A_{r} & =0.40 \\ F_{a} & =0.90 \\ F_{c} & =F_{R}=F_{L}=0.90 \\ h_{D} & =1.0 \mathrm{~cm} \\ K_{m} & =2.26 \\ f & =3.41 \mathrm{MHz} \\ K & =3.5 \times 10^{-8} \mathrm{~cm}^{-1} \\ \rho & =1.62 \times 10^{-6} \Omega \mathrm{cm}^{-1}(\mathrm{Ag}) \\ r & =1640 \mathrm{rad} \mathrm{oerstedt}^{-1} \mathrm{sec}^{-1} \\ t_{p} & =250 \mathrm{usec}\end{array}$

we have 


$$
\text { F-6 }
$$

\begin{tabular}{|c|c|c|c|c|c|}
\hline$r$ & 10 & 15 & 20 & 25 & 30 \\
\hline$n$ & 11.30 & 9.871 & 8.969 & 8.326 & 7.835 \\
\hline$S_{c}$ & $3.185 \times 10^{-2}$ & $3.646 \times 10^{-2}$ & $4.013 \times 10^{-2}$ & $4.323 \times 10^{-2}$ & $4.594 \times 10^{-2}$ \\
\hline$L$ & $49.67 \mu \mathrm{H}$ & 56.86 & 62.58 & 67.41 & 71.64 \\
\hline c & $43.85 \mathrm{pF}$ & 38.31 & 34.80 & 32.31 & 30.40 \\
\hline$\sigma$ & $34.69 \mu+$ & & & & \\
\hline$R$ & $0.1843 \Omega$ & 0.2415 & 0.2926 & 0.3395 & 0.3834 \\
\hline$Q$ & 5773 & 5043 & 4582 & 4254 & 4003 \\
\hline$H_{\text {MAX }}$ & 3.193 oe $\rightarrow$ & & & & \\
\hline$I_{\text {MAX }}$ & $15.26 \mathrm{~A}$ & 26.20 & 38.45 & 51.78 & 66.03 \\
\hline$V_{\text {MAX }}$ & $16.24 \mathrm{KV}$ & 31.92 & 51.56 & 74.79 & 101.3 \\
\hline$\left(P_{\text {MAX }}\right)_{\text {CIRC }}$ & $123.9 \mathrm{KW}$ & 418.2 & 991.2 & 1936 & 3345 \\
\hline$\left(P_{\text {MAX }}\right)_{D I S}$ & $21.47 \mathrm{~W}$ & 82.95 & 216.4 & 455.3 & 836.1 \\
\hline $\mathrm{F}_{\text {MAX }}$ & $4.511 \mathrm{kV} / \mathrm{cm}$ & 5.911 & 7.161 & 8.309 & 9.383 \\
\hline$E_{\text {MAX }}$ & $4.016 \mathrm{~mJ}$ & 13.55 & 32.13 & 62.75 & 108.4 \\
\hline$\left(P_{\text {MAX }}\right)_{\text {LOAD }}$ & $290.7 \mathrm{~W}$ & 981.1 & 2326 & 4542 & 7849 \\
\hline d & $3.185 \mathrm{~mm}$ & 5.469 & 8.026 & 10.81 & 13.78 \\
\hline 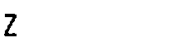 & $i 064 \Omega$ & 1218 & 1341 & 1444 & 1535 \\
\hline
\end{tabular}


G. Power Requirements in NQR Coil

The magnetic field generated on axis by a flat cotl is given by

$$
H=0.2 \pi \frac{n I r^{2}}{\left(r^{2}+x^{2}\right)^{2 / 3}}
$$

where

$$
\begin{aligned}
& H=\text { magnetic field, } \\
& \mathbf{n}=\text { number of turns } \\
& I=\text { current, } A \\
& r=\text { coil radius, } \mathrm{cm} \\
& X=\text { axial distance from plane of coil, cm }
\end{aligned}
$$

and its inductance by

$$
\mathbf{L}=\mathrm{Krn}^{2}
$$

where

$$
\begin{aligned}
& L=\text { self-inductance, } \mu H \\
& K=\text { coil constant }
\end{aligned}
$$

provided the number of turns on the conductor diameter are low. Since $L$ is limited by coil resonance considerations, we can substitute it in $H$ to give

$$
H=\frac{0.2 \pi}{K^{1 / 2}} \frac{I L^{1 / 2}}{\left(r+x^{2} / r\right)^{2 / 3}}
$$

In order to optimize $r$ for a given $x$, we take

$$
\frac{\partial H}{\partial r}=\frac{0.3 \pi}{\sqrt{K}} I \sqrt{L} \quad \frac{1-x^{2} / r^{2}}{\left(r+x^{2} / r\right)^{5 / 2}}
$$


and, for

$$
\frac{\partial H}{\partial r} \equiv 0 \quad \therefore \mathrm{r}=\mathrm{x}
$$

Since

$$
\frac{\partial^{2}-11}{\partial r^{2}}=-\frac{0.6 \pi}{K^{1 / 2}}, \bar{L}\left[\frac{-\left(r+x^{2} / r\right) x^{2} / r^{3}-\frac{1}{10}\left(1-x^{2} / r^{2}\right)^{2}}{\left(r+x^{2} / r\right)^{7 / 2}}\right]
$$

we have

$$
\left(\frac{\partial^{2} H}{\partial r^{2}}\right)_{r=x}>0
$$

thus showing the point $r=x$ to be a maximum, at which

$$
\mathrm{H}=0.222 \frac{\mathrm{I}^{1 / 2}}{\mathrm{~K}^{1 / 2} \mathrm{r}^{3 / 2}}
$$

a value only $2^{3 / 2}$ times lower than at the center of the coil. For a given $H$, then

$$
\mathrm{I}=4.50 \frac{\mathrm{Hr}^{3 / 2} \mathrm{~K}^{1 / 2}}{\mathrm{~L}^{1 / 2}}
$$

and since

$$
P=\left(\frac{I}{Q}\right)^{2} \Omega
$$


we have

$$
P=20.26 \frac{H^{2} r^{3} \Omega K}{L Q^{2}}
$$

where

$$
\begin{aligned}
& \Omega=\operatorname{coil} \text { reactance } \\
& Q=\text { coil } Q-\text { factor }
\end{aligned}
$$

The average power will be given by

$$
\bar{P}=P_{\mu} R_{p}
$$

where

$$
\begin{aligned}
& \mu \approx \text { pulse repetition rate, } \mathrm{HZ} \\
& R_{p}=\text { pulse repetition rate, } \mathrm{Hz}
\end{aligned}
$$

An example will now be instructive. Given

$$
\begin{aligned}
& H=40 \\
& r=10 \mathrm{~cm} \\
& \Omega=10^{5} \Omega \\
& L=40 \mu \mathrm{H} \\
& K=0.04 \\
& Q=100 \\
& \mu=160 \mu \mathrm{sec} \\
& R_{p}=2 \mathrm{~Hz}
\end{aligned}
$$

we have

$$
P=324 \mathrm{~kW}
$$

and

$$
\bar{p}=104 \mathrm{~W}
$$

The values for the other variables are

$$
\begin{aligned}
& n=10 \\
& I=180 A
\end{aligned}
$$


Giving for the input current to the coil

$$
I / Q=1.8 \mathrm{~A}
$$

and for the drive voltage

$$
I \Omega / Q=180 \mathrm{kV}
$$

This requirement can be reduced by subdividing the exciting pulse into a pulse train (whose duration must be $\left\langle T_{1}\right.$ ). Then $P, I, I / Q$ and $I \Omega / Q$ can be divided by the number of pulses in the train. 


\section{H. MGR Signal Loss for Finite Length Excitation Pulses}

Given a single exciting pulse followed by measurement of the free induction decay, we may define

$\mathrm{T}_{2}=$ free induction decay time constant, sec

$T_{p}=$ pulse duration, sec

$\mathrm{t}=$ time, sec

$H(t)=$ magnetic field as a function of time, oerstedt

$\gamma=$ gyramagnetic ratio, rad $\times \sec ^{-1} \times$ oerstedt $^{-1}$

To give a maximum signal, a saturated pulse is required, for which

$$
\gamma \int_{0}^{T_{p}} H(t) d t=\frac{\pi}{2}
$$

For such a pulse the fraction of the total pulse corresponding to a given instant is

$$
f(t)=\frac{2}{\pi} H(t) d t
$$

If the pulse duration is finite, part of the signal will decay during it; the fractional loss in intensity will be

$$
F=\int_{0}^{T_{p}} e^{-\left(T_{p}-t\right) / T_{2}} f(t)=\frac{2}{\pi} e^{-T_{p} / T_{2}} \int_{0}^{T_{p}} H(t) e^{-t / T_{2}} d t
$$


A few of the more typical cases can be tabulated (See Table $\mathrm{H}-1$ ). Figure $\mathrm{H}-1$ shows $F$ as a function of $T_{p}$ for all three cases and for $\alpha=1,2$, and 5 . If pulsing schemes requiring $q$ pulses within the thermal equilibration time are used, the actual $T_{p}$ must be multiplied by $q$ to get the effective one. 


\section{TABLE H- ?}

\begin{tabular}{|c|c|c|c|c|}
\hline PULSE SHAPE & $H(t)$ & CONSTANT & $F$ & $H_{\text {MAX }}$ \\
\hline SQUARE & C & $\frac{\pi}{2 \gamma T_{p}}$ & $\frac{T_{2}}{T_{p}}\left(1-e^{-T_{p} / T_{2}}\right)$ & $\frac{\pi}{2 \gamma \mathrm{T}_{\mathrm{p}}}$ \\
\hline TRIANGULAR & $\mathrm{Ct}$ & $\frac{\pi}{\gamma \mathrm{T}^{2}}$ & $2\left(\frac{T_{2}}{T_{p}}\right)^{2}\left(\frac{T_{p}}{T_{2}}-1+e^{-T_{p} / T_{2}}\right)$ & $\frac{\pi}{\gamma T_{p}}$ \\
\hline $\begin{array}{l}\text { EXPONENT IAL } \\
\text { DECAY }\end{array}$ & $C\left(1-e^{-\alpha t / T} p\right)$ & $\frac{\alpha \pi}{2 \gamma T_{p}\left(1-e^{-\alpha}\right)}$ & $\frac{T_{2} / T_{p} \alpha e^{-T_{p} / T_{2}}}{1-e^{-\alpha}} \frac{e^{-\alpha+T_{p} / T_{2}-1}}{1-\alpha T_{2} / T_{p}}$ & $\frac{\pi a}{2 \gamma T_{p}\left(1-c^{-a}\right)}$ \\
\hline $\begin{array}{l}\text { EXPONENTIAL- } \\
\text { ASYMPTOTIC }\end{array}$ & $C\left(e^{-\alpha t / T_{F}}\right.$ & $\frac{\alpha \pi}{2 \gamma T_{p}\left(\alpha+e^{-\alpha}-1\right)}$ & $\frac{T_{2} / T_{p} \alpha e^{-T_{p} / T_{2}}}{\alpha+e^{-\alpha}-1}\left(e^{T_{p} / T_{2}}-1+\frac{1-e^{-\alpha+T_{p} / T_{2}}}{1-\alpha^{2} / T_{p}}\right)$ & $\frac{\pi \alpha\left(1-e^{-a}\right)}{2 \gamma T_{p}\left(a+e^{-a}-1\right)}$ \\
\hline
\end{tabular}




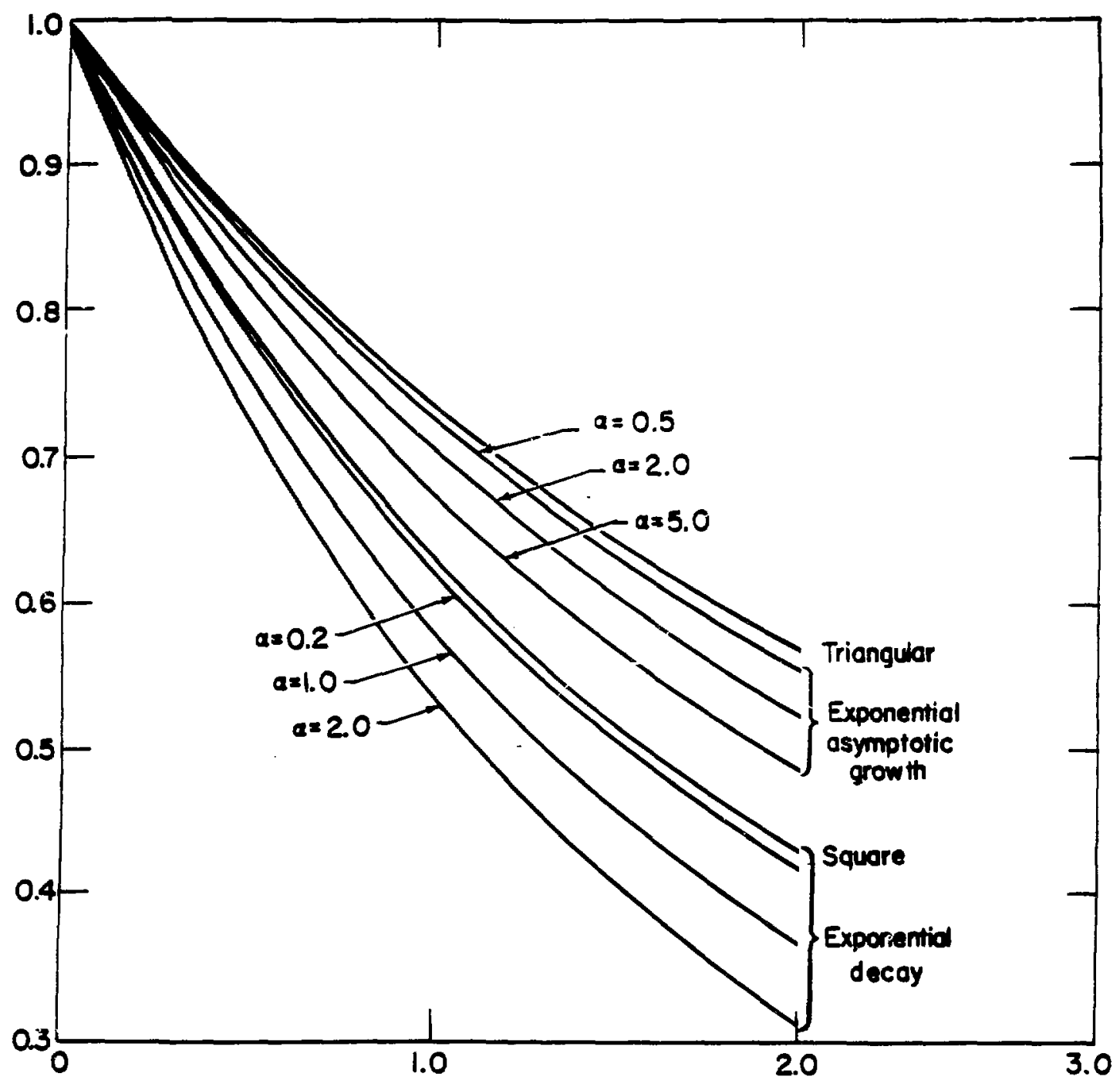

Figure H-1. Signal Loss fr. Finite Excitation Pulse Length 